\title{
Competition of Superconductivity and Charge Density Waves in Cuprates: Recent Evidence and Interpretation
}

\author{
A. M. Gabovich, ${ }^{1}$ A. I. Voitenko, ${ }^{1}$ T. Ekino, ${ }^{2}$ Mai Suan Li, ${ }^{3}$ H. Szymczak, ${ }^{3}$ and M. Pȩkała ${ }^{4}$ \\ ${ }^{1}$ Institute of Physics, National Academy of Sciences of Ukraine, 46 Nauka Avenue, Kyiv 03680, Ukraine \\ ${ }^{2}$ Graduate School of Integrated Arts and Sciences, Hiroshima University, Higashi-Hiroshima 739-8521, Japan \\ ${ }^{3}$ Institute of Physics, Al. Lotników 32/46, 02-668 Warsaw, Poland \\ ${ }^{4}$ Department of Chemistry, University of Warsaw, Al. Żwirki i Wigury 101, 02-089 Warsaw, Poland \\ Correspondence should be addressed to A. M. Gabovich, alexander.gabovich@gmail.com
}

Received 2 June 2009; Accepted 1 September 2009

Academic Editor: Sasha Alexandrov

Copyright (๑) 2010 A. M. Gabovich et al. This is an open access article distributed under the Creative Commons Attribution License, which permits unrestricted use, distribution, and reproduction in any medium, provided the original work is properly cited.

\begin{abstract}
Explicit and implicit experimental evidence for charge density wave (CDW) presence in high- $T_{c}$ superconducting oxides is analyzed. The theory of CDW superconductors is presented. It is shown that the observed pseudogaps and dip-hump structures in tunnel and photoemission spectra are manifestations of the same CDW gapping of the quasiparticle density of states. Huge pseudogaps are transformed into modest dip-hump structures at low temperatures, $T$, when the electron spectrum superconducting gapping dominates. Heat capacity jumps at the superconducting critical temperature and the paramagnetic limit are calculated for CDW superconductors. For a certain range of parameters, the CDW state in a $d$-wave superconductor becomes reentrant with $T$, the main control quantity being a portion of dielectrcally gapped Fermi surface. It is shown that in the weak-coupling approximation, the ratio between the superconducting gap at zero temperature $\Delta(T=0)$ and $T_{c}$ has the Bardeen-Cooper-Schrieffer value for $s$-wave Cooper pairing and exceeds the corresponding value for $d$-wave pairing of CDW superconductors. Thus, large experimentally found values $2 \Delta(T=0) / T_{c} \approx 5 \div 8$ are easily reproduced with reasonable input parameter values of the model. The conclusion is made that CDWs play a significant role in cuprate superconductivity.
\end{abstract}

\section{Introduction}

Ever since the earliest manifestations of high- $T_{c}$ superconductivity were found in 1986 [1], the whole theoretical power [2-22] has been applied to explain and describe various normal and superconducting properties of various oxide families with critical temperatures, $T_{c}$, ranging up to $138 \mathrm{~K}$ to date [23-27]. Unfortunately, even conceptual understanding of the mechanisms and character of superconductivity in cuprates is still lacking. Strictly speaking, there is a number of competing paradigms, every of them pretending to be "the theory of superconductivity" (see, e.g., [2]) but not recognized as such by other respected experts in the field.

After the discovery of high- $T_{c}$ oxides, experimentalists found several other superconducting families with $T_{c}$ higher than $23.2 \mathrm{~K}$ reached by the precuprate record-holder, $\mathrm{Nb}_{3} \mathrm{Ge}$ $[28,29]$. For instance, one may refer to fullerides $[30,31]$, doped bismuthates [32-34], hafnium nitrides [35, 36], and magnesium diborides [37-40]. One should also mention more controversial cases of superconducting oxides $\mathrm{H}_{x} \mathrm{WO}_{3}$ with $T_{c} \approx 120 \mathrm{~K}[41]$ and $\mathrm{Sr}_{0.9} \mathrm{La}_{0.1} \mathrm{PbO}_{3-\delta}$ with $T_{c} \approx$ $65 \mathrm{~K}$ [42]. Finally, an unexpected and counter-intuitive discovery of the iron-based oxypnictide $[43,44]$ or oxygenfree pnictide [45] layered superconductors with $T_{c}$ over $50 \mathrm{~K}$ has been made recently (see also reviews [46-49]).

Presumably, the latter materials with FeAs layers have been overlooked as possible candidates for high- $T_{c}$ superconductors, since Fe ions in solids usually possess magnetic moments, which promote magnetic ordering, the latter being detrimental to superconductivity, especially the spinsinglet one [50-55]. Strictly speaking, such an omission is of no surprise because superconductivity in oxides is rather gentle, sensible to impurities, including the excess or deficiency of oxygen [56] in these nonstoichiometric $[57,58]$ 
compounds. Recent discovery [59] of previously unnoticed high- $T_{c}$ superconductivity in parent compounds $T^{\prime}-R_{2} \mathrm{CuO}_{4}$ $(R=\mathrm{Pr}, \mathrm{Nd}, \mathrm{Sm}, \mathrm{Eu}, \mathrm{Gd})$ is very symptomatic in this regard, since an accurate removal of apical oxygen from thin films raised $T_{c}$ from exact zero (those compositions were earlier considered by theoreticians as typical correlated Mott-Hubbard insulators) to $32.5 \mathrm{~K}$ for $\mathrm{Nd}_{2} \mathrm{CuO}_{4}$. As for the ferroarsenide family, one of its members, $\mathrm{EuFe}_{2}\left(\mathrm{As}_{0.7} \mathrm{P}_{0.3}\right)_{2}$, reveals a true superconducting transition at $26 \mathrm{~K}$, followed by the ferromagnetic ordering of $\mathrm{Eu}^{2+}$ magnetic moments below $20 \mathrm{~K}$, coexisting with superconductivity [60], which is quite unusual in view of the antagonism indicated above between two kinds of cooperative phenomena.

What is more, none of the mentioned superconductors except $\mathrm{Ba}_{1-x} \mathrm{~K}_{x} \mathrm{BiO}_{3}$ [33] were discovered due to theoretical predictions. Hence, one may consider the theoretical discovery of $\mathrm{Ba}_{1-x} \mathrm{~K}_{x} \mathrm{BiO}_{3}$ as an accidental case, since, according to the well-known chemist Cava: "one of the joys of solid state chemistry is its unpredictability" [61]. The same opinion was expressed by the other successive chemist Hosono: "understanding the mechanism with respect to predicting the critical temperature of a material is far from complete at the present stage even for brilliant physicists. Such a situation provides a large opportunity including a good luck for material scientists who continue the exploration for a new material, not limited to superconductors, and a new functionality based on their own view points" [48]. That is why Pickett recently made a sad remark that "the next breakthrough in superconductivity will not be the result of surveying the history of past breakthroughs" [62]. It means that microscopic theories of superconductivity are incapable of describing specific materials precisely, although together they give an adequate overall picture. In this connection, the failure of the most sophisticated approaches to make any prediction of true or, at least "bare" $T_{c}$, (provided that the corresponding $T_{c}$-value is not known a priori) despite hundreds of existing superconductors with varying fascinating properties, forced Phillips [63] to reject all apparently first-principle continuum theories in favor of his own percolative filamentary theory of superconductivity [64-67] (see also the random attractive Hubbard model studies of superconductivity $[68,69]$ and the analysis of competition between superconductivity and charge density waves studied in the framework of similar scenarios [70-72]). We totally agree with such considerations in the sense of the important role of disorder in superconductors with high $T_{c}$ on the verge of crystal lattice instability [73-83]. Nevertheless, it is questionable whether a simple one-parameter "master function" of $[63,67]$ would be able to make quantitative and practically precise predictions of $T_{c}$. As for the qualitative correctness of the dependence $T_{c}$ versus weighted number $\langle R\rangle$ of Pauling resonating valence bonds $[63,67,84]$, it can be considered at least as a useful guideline in the superconductivity ocean. The phenomenological character of the master function (chemical trend diagram) $T_{c}(\langle R\rangle)$ is an advantage rather than a shortcoming of this approach, as often happens in the physics of superconductors (see, e.g., more or less successful criteria of superconductivity with different extent of phenomenology [85-98]).
On the other hand, attempts to build sophisticated microscopic theories of the boson-mediated BardeenCooper-Schrieffer (BCS) attraction, treating the Coulomb repulsion as a single Coulomb pseudopotential constant $\mu^{*}$, are incapable of predicting actual critical superconducting properties [63, 91, 99-101]. The same can be said [67] about Hubbard-Hamiltonian models with extremely strong repulsive Coulomb energy parameter $U$, which is formally based on the opposite ideology (see, e.g., $[102,103])$. As an example of the theories described above, one can indicate work [104], where the strong-coupling Eliashberg equations for the electron-phonon mechanism of superconductivity $[105,106]$ were solved numerically taking into account even vertex corrections and treating the dispersive Coulomb interaction not on equal footing, but as a simple constant $\mu^{*}$. In this connection, it seems that the prediction of [104] that the maximal $T_{c}$ for new iron-based superconductors is close to $90 \mathrm{~K}$ is unjustified. Of course, the same is true for other studies of such a kind.

It is remarkable that, for hole- and electron-doped cuprates, there is still no clarity concerning the specific mechanisms of superconductivity $[17,107-115]$ and the order parameter symmetry $[109,116-130]$, contrary to the "official" viewpoint [131-133]) and even the very character of the phenomenon (in particular, there have been furious debates concerning the Cooper pairing versus boson condensation dilemma in cuprates $[8,134,135])$. The same seems to be true for other old and new "exotic" superconductors $[46,107,108,136-153]$, their exoticism being in essence a degree of our ignorance.

It would be of benefit to consider all indicated problems in detail for all classes of superconductors and show possible solutions. Unfortunately, it cannot be done even in the scope of huge treatises (see, e.g., [154-157]). The objective of this review is much more modest. Specifically, it deals mostly with high- $T_{c}$ cuprate materials, other superconductors being mentioned only for comparison. Moreover, in the present state of affairs, it would be too presumptuous to pretend to cover all aspects of the oxide superconductivity. Hence, we will restrict ourselves to the analysis of lattice instabilities and concomitant charge density waves (CDWs) in high$T_{c}$ oxides. Their interplay with superconductivity is one of the fascinating and fundamentally important phenomena observed in cuprates and discussed by us earlier [158-160]. Nevertheless, in this rapidly developing branch of the solid state physics, many new theories and experimental data on various CDW superconductors appeared during last years. They are waiting for both unbiased and thorough analysis. This article discusses this new information, referring the reader to our previous reviews for more general and established issues, as well as some cumbersome technical details.

The outline of this review is as follows. In Section 2, for the sake of completeness, we briefly consider possible mechanisms of superconductivity in cuprates, the problem of the relationship between BCS pairing and BoseEinstein condensation (BEC), and the multigapness of the superconducting order parameter. Section 3 is devoted to the experimental evidence for CDWs, the so-called 
pseudogaps, dip-hump structures, and manifestations of intrinsic inhomogeneity in cuprate materials. The original theory of CDW superconductors and the interpretation of CDW-related phenomena in high- $T_{c}$ oxides are presented in Section 4. At the end of Section 4, some recent data on coexistence between superconductivity and spin density waves (SDWs) - a close analogue of CDWs - are covered. This topic became hot once more after the discovery of ferropnictides [43-48, 161]. Short conclusions are made in Section 5.

\section{Considerations on Peculiarities and Mechanisms of Superconductivity in Oxides}

When $\mathrm{BaPb}_{1-x} \mathrm{Bi}_{x} \mathrm{O}_{3-\delta}(\mathrm{BPB})$ was shown [162] to be a superconductor with a huge (at that time!) $T_{c} \approx 13 \mathrm{~K}$ for $x \approx 0.25$, a rather low concomitant concentration of current carriers $n \approx 1.5 \div 4.5 \times 10^{21} \mathrm{~cm}^{-3}$, and poor electric conductivity [56] (the phase diagram of BPB is extremely complex, with a number of partial metal-insulator structural transitions [56, 163-167]), it looked like an exception. Now, it is fully recognized that oxides with highest $T_{c}$ are bad metals from the viewpoint of normal state conductivity [168]. In particular, the mean free path of current carriers is of the order of the crystal lattice constant, so that the Ioffe-Regel criterion of the metal-insulator transition [169] is violated. Moreover, there exists an oxide superconductor $\mathrm{SrTiO}_{3-\delta}$ with a tiny maximal $T_{c} \approx 0.5 \mathrm{~K}$, attained by doping, but an extremely small $n<10^{20} \mathrm{~cm}^{-3}$ [170]. Note that the undoped semiconducting $\mathrm{SrTiO}_{3-\delta}$ is so close to the metal-insulator border that it may be transformed into a metal by the electrostatic-field effect [171] (this technique has been successfully applied to other oxides [172]). Moreover, a two-dimensional metallic layer has been discovered [173] at the interface between two insulating oxides $\mathrm{LaAlO}_{3-\delta}$ and $\mathrm{SrTiO}_{3-\delta}$, which was later found to be superconducting with $T_{c} \approx 0.2 \mathrm{~K}[174,175]$. The appearance of superconductivity at nonmetallic charge carrier densities in oxides of different classes comprises a hint that it is not wise to treat various oxide families separately (see, e.g., [176]), all of them having similar perovskitelike ion structures $[23,25,26,177-181]$ and similar normal and superconducting properties [27], whatever the values of their critical parameters are. As for the apparent dispersion of the latter among superconducting oxides, it mostly reflects their conventional exponential dependences on atomic and itinerant-electron characteristics $[9,10]$.

The junior member of the superconducting oxide family, $\mathrm{SrTiO}_{3-\delta}$, demonstrates (although not in a spectacular manner) several important peculiarities, which are often considered as properties intrinsic primarily to high- $T_{c}$ cuprates. Indeed, in addition to the low $n$, this polar, almost ferroelectric [182, 183], material was shown to reveal polaron conductivity [184] and is suspected to possess bipolaron superconductivity [185-187], first suggested by Vinetskii almost 50 years ago [188]. It means that $\mathrm{SrTiO}_{3-\delta}$ might be not a Bardeen-Cooper-Schrieffer (BCS) superconductor [189] with a large coherence length $\xi_{0} \gg a_{0}$, where $a_{0}$ is the crystal lattice constant, but most likely an example of a material with $\xi_{0} \simeq a_{0}$, so that a Bose-condensation of local electron pairs would occur at $T_{c}$, according to the Schafroth-Butler-Blatt scenario [190] or its later extensions [8, 134, 135, 191-199].

The concept of bipolarons (local charge carrier pairs) has been later applied to $\mathrm{BPB}$ [200-203], $\mathrm{Ba}_{1-x} \mathrm{~K}_{x} \mathrm{BiO}_{3-\delta}$ (BKB, $\left.T_{c} \leq 30 \mathrm{~K}[204,205]\right)[203,206,207]$ and cuprates [195, 199, 208-211]. It was explicitly shown for BPB and BKB by Xray absorption spectroscopy [203] that bipolaronic states and CDWs coexist and compete, which might lead, in particular, to the observed nonmonotonic dependence $T_{c}(x)$ [212]. At the same time, Hall measurements demonstrate that the more appropriate characteristics $T_{c}(n)$ is monotonic [56, $213,214]$, so that the expected suppression of $T_{c}$ at high $n$ as a consequence of screening of the electron-phonon matrix elements $[99,215,216]$ is not achieved here as opposed to the curve $T_{c}(n)$ [170] in reduced samples of $\mathrm{SrTiO}_{3-\delta}$. As for cuprates, the bipolaron superconductivity mechanism, as well as any other BEC scheme, in its pure state would require an existence of the preformed electron (hole) pairs (bipolarons), which might be the case [177, 217], and a prior destruction of the Fermi surface (FS), the condition contradicting observations (see, e.g., [218]). Therefore, bosonfermion models for charge carriers in superconductors was introduced [134, 219-225] and, later on, severely criticized $[226,227]$. In any case, the available objections concern the bipolaronic mechanism of superconductivity itself, the occurrence of polaronic effect in oxides with high dielectric permittivities raising no doubt [115, 177, 199, 228-232].

It is remarkable that the boson-fermion approach mentioned above is not a unique tool for describing superconductivity in complex systems. A necessary "degree of freedom" connected to another group of charge carriers has been introduced, for example, as the so-called $(-U)$ centers [233-235], earlier suggested by Anderson [236] as a phenomenological reincarnation of bipolarons in amorphous materials [188]. Independently, narrow-band nondegenerate charge carriers submerged into the sea of itinerant electrons were proposed for cuprates as another, not fully hybridized kind of the "second heavy component" [237, 238]. For completeness, we should also mention a quite different model involving a second heavy charge carrier subsystem ( $d$-electrons in transition metals [239] or heavy holes in degenerate semiconductors [240]), necessary to convert high-frequency Langmuir plasmons intrinsic to the itinerant electron component into the ion-acoustic collective excitation branch, in order that a high- $T_{c}$ superconductivity would appear. Those hopes, however, lack support from any evidence in natural or artificial systems (see the analysis of plasmon mechanisms [206, 241-247], the optimism of some authors seems to us and others [248] a little bit exaggerated). As can be readily seen from the References given above, all nonconventional approaches, rejecting or generalizing the BCS scheme and going back to the explanations of a relatively weak superconductivity in degenerate semiconductors [138, 191, 215, 249-252], have been applied to every family of superconducting oxides, including cuprates. 
Strontium titanate became a testing ground [253] of one further attractive idea (based on the same concept of several interacting charge carrier components) of twogap or multigap superconductivity, with the interband interplay being crucial to the substantial increase of $T_{c}$ and other critical parameters. The corresponding models came into being in connection with the transition $s$ - $d$ metals $[254,255]$. They were subsequently applied to analyze superconductivity in multivalley semiconductors [256, 257], high- $T_{c}$ oxides [231, 258-266], $\mathrm{MgB}_{2}$ [40, 267-269], $\mathrm{ZrB}_{12}$ [270], $\mathrm{V}_{3} \mathrm{Si}$ [271], $\mathrm{Mg}_{10} \mathrm{Ir}_{19} \mathrm{~B}_{16}$ [272], $\mathrm{YNi}_{2} \mathrm{~B}_{2} \mathrm{C}$ [273], $\mathrm{NbSe}_{2}$ [274, 275], $R_{2} \mathrm{Fe}_{3} \mathrm{Si}_{5}(R=\mathrm{Lu}, \mathrm{Sc})$ [276], $\mathrm{Sc}_{5} \mathrm{Ir}_{4} \mathrm{Si}_{10}$ [277], $\mathrm{Na}_{0.35} \mathrm{CoO}_{2} \cdot 1.3 \mathrm{H}_{2} \mathrm{O}[278]$ as well as pnictides $\mathrm{LaFeAsO}_{1-x} \mathrm{~F}_{x}$ [279], $\mathrm{LaFeAsO}_{0.9} \mathrm{~F}_{0.1}$ [280], $\mathrm{SmFeAsO}_{0.9} \mathrm{~F}_{0.1}$ [281], and $\mathrm{Ba}_{0.55} \mathrm{~K}_{0.45} \mathrm{Fe}_{2} \mathrm{As}_{2}$ [282], $\mathrm{Ba}_{1-x} \mathrm{~K}_{x} \mathrm{Fe}_{2} \mathrm{As}_{2}$ with $T_{c} \simeq 32 \mathrm{~K}$ [283]. We did not explicitly include into the list such modifications of magnesium diboride as $\mathrm{Mg}_{1-x} \mathrm{Al}_{x} \mathrm{~B}_{2}$ or $\operatorname{Mg}\left(\mathrm{B}_{1-x} \mathrm{C}_{x}\right)_{2}$, and so forth.

Since, instead of one, two or more well-separated superconducting energy gaps, a continuous, sometimes wide, gap distribution is often observed (see results for $\mathrm{Nb}_{3} \mathrm{Sn}$ in [284] and $\mathrm{MgB}_{2}$ in [285-289]), the original picture of the gap multiplicity in the momentum, $\mathbf{k}$, space loses its beauty, whereas the competing scenario $[76,290]$ of the spatial (r-space) extrinsic or intrinsic gap spread becomes more adequate and predictive [77-79]. For the case of cuprates, it has been recently shown experimentally that the spread is really spatial, but corresponds to the pseudogap (CDW gap) rather than its superconducting counterpart, the latter most probably being a single one [291] (see also the discussion in [83] and below).

In accordance with what was already mentioned, the application of very different, sometimes conflicting, models to oxide families, including cuprates, means an absence of a deep insight into the nature of their superconducting and normal state properties. We are not going to analyze here the successes and failures of the microscopic approaches to high$T_{c}$ superconductivity in detail; instead we want to emphasize that even the boson-mediators (we accept the applicability of the Cooper-pairing concept to oxides on the basis of crucial flux-quantization experiments [292, 293]) are not known for sure. Indeed, at the early stages of the high- $T_{c}$ studies, magnons were considered as glue, coupling electrons or holes. The very temperature-composition (doping) phase diagrams supported this idea, since undoped and slightly doped oxides were found antiferromagnetic [26, 103, 294304]. However, a plethora of theories suggesting virtual spin fluctuations as the origin of superconductivity in high- $T_{c}$ oxides and leading to the $d_{x^{2}-y^{2}}$ symmetry of the superconducting order parameter have been developed [6, $11,15,103,302,305-310]$.

Fortunately for the scholars, it became clear that reality is richer for oxides than was expected, so that (i) the order parameter may include a substantial $s$-wave admixture [109, 116-129]; and (ii) phonons still exist in perovskite crystal lattices, inevitably affecting or, may be, even determining the pairing process $[4,10,112,115,311]$, not to talk about polaron and bipolaron effects discussed above. It should be noted that there are reasonable scenarios of $d$-wave order parameter symmetry in the framework of the electron-phonon interaction alone [208, 312-316] (a similar conclusion was made for the case of plasmon mechanism [317]).

At the same time, if one adopts a substantial (crucial?) role of spin-fluctuation mechanism in superconductivity, the ubiquitous phonons can (i) be neutral to the dominant $d$ wave pairing; (ii) act synergetically with spin fluctuations; (iii) or reduce $T_{c}$, as it would have been for switched-off phonons. The existing theories support all three variants, although some authors cautiously avoid any direct conclusions [103]. For instance, Kulić demonstrated the destructive interference between both mechanisms of superconductivity [4]. Phononic reduction of the magnetically induced $T_{c}$ was claimed in $[308,318]$, whereas anisotropic phonons seem to enhance $T_{c}$, thus obtained [319]. Finally, according to [228, $320,321]$, spins and phonons act constructively in cuprates. Once again, the microscopic approach was incapable of unambiguously predicting a result for the extremely complex system.

One should bear in mind that the problem is much wider than the interplay between spin excitations and phonons. Namely, it is more correct to consider the interplay between Coulomb inter-electron and electron-lattice interactions $[232,322]$. Of course, the latter is also Coulombic in nature, phonons being simply an ion sound, that is, ion Langmuir plasma oscillations [323] screened in this case by degenerate light electrons [324] (thus, acoustic phonons constitute a similar phenomenon as the acoustic plasmons in the electron system $[239,240]$ with an accuracy to frequencies). One of the main difficulties is how to separate the metal constituents in order that some contributions would not be counted twice $[322,325-333]$. Since it is possible to do rigorously only in primitive plasma-like models [91, 92, 99, 251, 322], the problem has not been solved. Therefore, empirical considerations remain the main source of future success for experimentalists, as it happened, for example, in the case of $\mathrm{MgB}_{2}$ [37].

\section{CDWs and CDW-Related Phenomena in Cuprates}

The reasoning presented in Section 2 demonstrates that for the objects concerned, it is insufficient to rely only on microscopic theories, so that phenomenological approaches should deserve respect and attention. In actual truth, they might not be less helpful in understanding the normal and superconducting properties of cuprates, being generalizations of a great body of experimental evidence collected during last decades. In this section, we are going to show that two very important features are common to all high- $T_{c}$ families. Specifically, these are the intrinsic inhomogeneity of nonstoichiometric superconducting ceramic and single crystalline samples [334-343] and the persistence of CDWs $[340,344,345]$ and other phenomena, which we also consider as CDW manifestations (dip-hump structures, DHS [339, 346-348], and pseudogaps below and above $T_{c}$ [349358 ] in tunneling spectra and angle-resolved photoemission spectra, ARPES). 


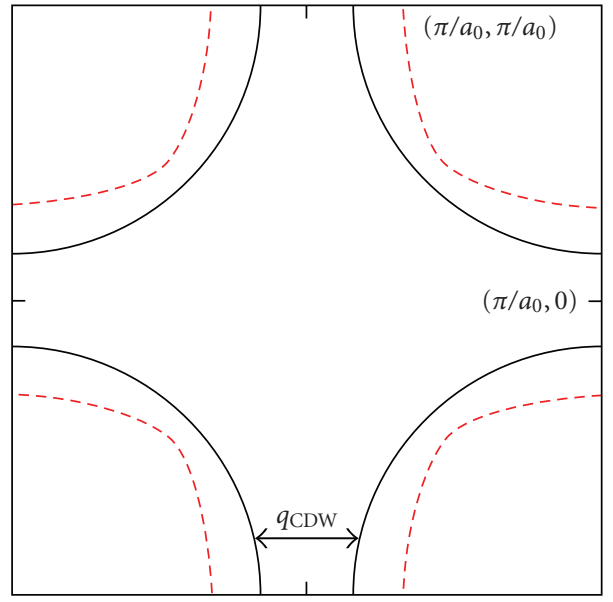

Figure 1: (Color online) Fermi surface nesting; and tight-bindingcalculated Fermi surface (solid black curve) of optimally doped $\mathrm{Bi}_{2} \mathrm{Sr}_{2} \mathrm{CuO}_{6+\delta}$ based on ARPES data [373]. The nesting wave vector (black arrow) in the antinodal flat band region has length $2 \pi / 6.2 a_{0}$. Underdoped $\mathrm{Bi}_{2} \mathrm{Sr}_{2} \mathrm{CuO}_{6+\delta}$ Fermi surfaces (shown schematically as red dashed lines) show a reduced volume and longer nesting wave vector, consistent with a CDW origin of the doping-dependent checkerboard pattern reported here (Taken from [344]).

CDWs were seen directly as periodic incommensurate structures in superconducting $\mathrm{Bi}_{2} \mathrm{Sr}_{2} \mathrm{CaCu}_{2} \mathrm{O}_{8+\delta}$ (BSCCO) using various experimental methods [12, 334, 359-370]. Photoemission studies reveal the $4 a_{0} \times 4 a_{0}$ charge-ordered "checkerboard" state in $\mathrm{Ca}_{2-x} \mathrm{Na}_{x} \mathrm{CuO}_{2} \mathrm{Cl}_{2}$ [371], and tunnel measurements visualized the same kind of ordering in BSCCO [370]. Scanning tunnel microscopy (STM) measurements found CDWs in $\mathrm{Bi}_{2} \mathrm{Sr}_{1.4} \mathrm{La}_{0.6} \mathrm{CuO}_{6+\delta}\left(T_{c}^{\max } \approx\right.$ $29 \mathrm{~K}$ ) with an incommensurate period and CDW wave vectors $\mathbf{Q}$ depending on oxygen doping degree [340]. The same method revealed nondispersive (energy-independent) checkerboard CDWs in $\mathrm{Bi}_{2-y} \mathrm{~Pb}_{y} \mathrm{Sr}_{2-z} \mathrm{La}_{z} \mathrm{CuO}_{6+x}\left(T_{c} \approx 35 \mathrm{~K}\right.$ for the optimally doped composition) [344]. In this case, $\mathbf{Q}$ substantially depends on doping, rising from $\pi a_{0}^{-1} / 6.2$ in an optimally doped sample to $\pi a_{0}^{-1} / 4.5$ for an underdoped sample with $T_{c} \approx 25 \mathrm{~K}$. It is easily explained by the authors taking into account the shrinkage of the hole FS with decreasing hole number, so that the vector $\mathbf{Q}$ that links the flat nested FS sections grows, whereas the CDW period decreases (see Figure 1). One should note that, in the presence of impurities (e.g., an inevitably non-homogeneous distribution of oxygen atoms), the attribution of the observed charge order (if any) to unidirectional or checkerboard type might be ambiguous [372].

A similar coexistence of CDWs and superconductivity was observed in a good many different kinds of materials with a reduced dimensionality of their electron system, so that the corresponding FS includes nested (congruent) sections [158-160]. For completeness, we will add some new cases discovered after our previous reviews were published. First of all, the analogy between CDWs in cuprates and layered dichalcogenides was proved by ARPES [352, 374376]. It should be noted that CDW competition with superconductivity in cuprates was supposed as early as in 1987 on the basis of heat capacity and optical studies [377], whereas the similarity between high- $T_{c}$ oxides and dichalcogenides was first noticed by Klemm [378, 379]. Additionally, a new dichalcogenide system $\mathrm{Cu}_{x} \mathrm{TiSe}_{2}$ was found with coexisting superconductivity and CDWs (at $0.04<x<0.06)[380,381]$. The coexistence between two phenomena was observed in the organic material $\alpha$-(BEDT$\mathrm{TTF})_{2} \mathrm{KHg}(\mathrm{SCN})_{4}$, but superconductivity was attributed to boundaries between CDW domains, where the CDW order parameter is suppressed [382]. High-pressure studies of another organic conductor $(\mathrm{Per})_{2}\left[\mathrm{Au}(\mathrm{mnt})_{2}\right]$ revealed an appearance of superconductivity after the CDW suppression [383]. Still, it remained unclear, whether some remnants of CDWs survived in the superconducting region of the phase diagram. Application of high pressure also suppressed CDWs in the compound $\mathrm{TbTe}_{3}$ at about $P=2.3 \mathrm{GPa}$, inducing superconductivity with $T_{c} \approx 1.2 \mathrm{~K}$, enhanced to $4 \mathrm{~K}$ at $P=$ 12.4 GPa [384], the behavior demonstrating the competition of Cooper and electron-hole pairings for the FS [385, 386]. The same experiments in this quasi-two-dimensional material revealed two kinds of CDW anomalies merging at $P=2.3 \mathrm{GPa}$, as well as antiferromagnetism, which makes this object especially promising. Finally, CDWs were found in another superconducting oxide $\mathrm{Na}_{0.3} \mathrm{CoO}_{2} \cdot 1.3 \mathrm{H}_{2} \mathrm{O}$ by specific heat investigations [387-389], showing two-energygap superconductivity for as-prepared samples and nonsuperconducting CDW dielectrized state after ageing of the order of days. The sample ageing is a situation widely met for superconductors [390, 391], whereas the dielectrization of as-synthesized superconducting ceramic samples accompanied by a transformation of bulk superconductivity into a percolating one with the CDW background was observed for BPB long ago $[56,392]$. Nevertheless, such a scenario was not proved directly at that time, while the bulk heat capacity peak in $\mathrm{Na}_{0.3} \mathrm{CoO}_{2} \cdot 1.3 \mathrm{H}_{2} \mathrm{O}$ [387-389] unequivocally shows the emergence of CDWs instead of superconductivity.

We emphasize that CDWs compete with superconductivity, whenever they meet on the same FS. This is the experimental fact, which agrees qualitatively with a number of theories [385, 386, 393-397].

Returning to cuprates, we want to emphasize that the existence of pseudogaps above and below $T_{c}$ is one of their most important features. Pseudogap manifestations are diverse, but their common origin consists in the (actually, observed) depletion of the electron densities of states (DOS). It is natural that tunnel and ARPES experiments, which are very sensitive to DOS variations, made the largest contribution to the cuprate pseudogap data base (see references in our works [81-83, 158-160]). Recent results show that the concept of two gaps (the superconducting gap and the pseudogap, the latter considered here as a CDW gap) $[82,352,353,357,377,398-404]$ begins to dominate in the literature over the one-gap concept [211, 355, 405-416], according to which the pseudogap phenomenon is most frequently treated as a precursor of superconductivity (for instance, a gas of bipolarons that Bose-condenses below $T_{c}$ [413] or a $d$-wave superconducting-like state without a long-range phase rigidity [416]). The main arguments, 
which make the one-gap viewpoint less probable, is the coexistence of both gaps below $T_{c}[349,417]$, their different position in the momentum space of the two-dimensional Brillouin zone [351, 353, 356, 418, 419], and their different behaviors in the external magnetic fields $\mathbf{H}$ [420], for various dopings [417], and under the effects of disordering [419].

Nevertheless, some puzzles still remain unresolved in the pseudogap physics. For instance, Kordyuk et al. [352] found that the pseudogap in $\mathrm{Bi}(\mathrm{Pb})_{2} \mathrm{Sr}_{2} \mathrm{Ca}(\mathrm{Tb}) \mathrm{Cu}_{2} \mathrm{O}_{8+\delta}$ revealed by ARPES is nonmonotonic in T. Such a behavior, as they indicated, might be related to the existence of commensurate and incommensurate CDW gaps, in a close analogy with the case of dicahlcogenides [421]. Another photoemission study of $\mathrm{La}_{1.875} \mathrm{Ba}_{0.125} \mathrm{CuO}_{4}$ has shown [354] that there seems to be two different pseudogaps: a $d$-wave-like pseudogapa precursor to superconductivity-near the node of the truly superconducting gap and a pseudogap in the antinodal momentum region - it became more or less familiar to the community during last years [350, 351, 353, 356, 403, 418, 419] and is identified by us as the CDW gap.

Despite existing ambiguities, the most probable scenario of the competition between CDW gaps (pseudogaps) and superconducting gaps in high- $T_{c}$ oxides, in particular, in BSCCO, includes the former emerging at antinodal (nested) sections of the FS and the latter dominating over the nodal sections (see Figure 2, reproduced from [403], where BSCCO was investigated, and results for $(\mathrm{Bi}, \mathrm{Pb})_{2}(\mathrm{Sr}, \mathrm{La})_{2} \mathrm{CuO}_{6+\delta}$ presented in [356]). Since CDW gaps are much larger than their superconducting counterparts, the simultaneous existence of the superconducting gaps in the antinodal region might be overlooked in the experiments. This picture means that the theoretical model of the partial dielectric gapping (of CDW origin or caused by a related phenomenon-spin density waves, SDWs) belonging to Bilbro and McMillan [385] (see also [56, 158-160, 386, 397, 422-428]) is adequate for cuprates. On the other hand, the coexistence of CDW and superconducting gaps, each of them spanning the whole FS [429-432], can happen only for extremely narrow parameter ranges [433]. Moreover, as is clearly seen from data presented in Figure 2 [403] and a lot of other measurements for different classes of superconductors, complete dielectric gapping has not been realized. The reason is obvious: nested FS sections cannot spread over the whole FS, since the actual crystal lattice is always three-dimensional and three-dimensionality effects lead to the inevitable FS warping detrimental to nesting conditions formulated below.

It is interesting that pseudogaps were also observed in oxypnictides $\mathrm{LaFeAsO}_{1-x} \mathrm{~F}_{x}$ and $\mathrm{LaFePO}_{1-x} \mathrm{~F}_{x}$ by ARPES [434] and $\mathrm{SmFeAsO}_{0.8} \mathrm{~F}_{0.2}$ by femtosecond spectroscopy [435], where SDWs might play the same role as CDWs do in cuprates. At the same time, in iron arsenide $\mathrm{Ba}_{1-x} \mathrm{~K}_{x} \mathrm{Fe}_{2} \mathrm{As}_{2}$, photoemission studies detected a peculiar electronic ordering with a $\left(\pi / a_{0}, \pi / a_{0}\right)$ wave vector [436], a true nature of which is still not known, but which might be related either to the magnetic reconstruction of the electron subsystem (SDWs) and/or to structural transitions (when CDWs accompanied by periodic crystal lattice distortions emerge in the itinerant electron liquid near the structural transition

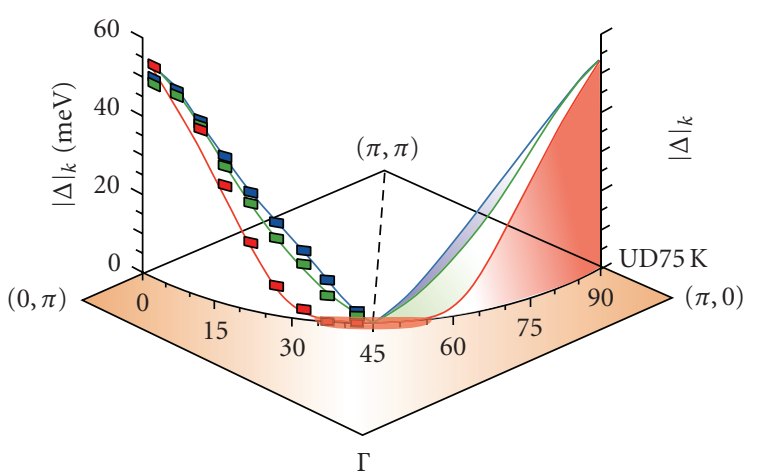

(a)

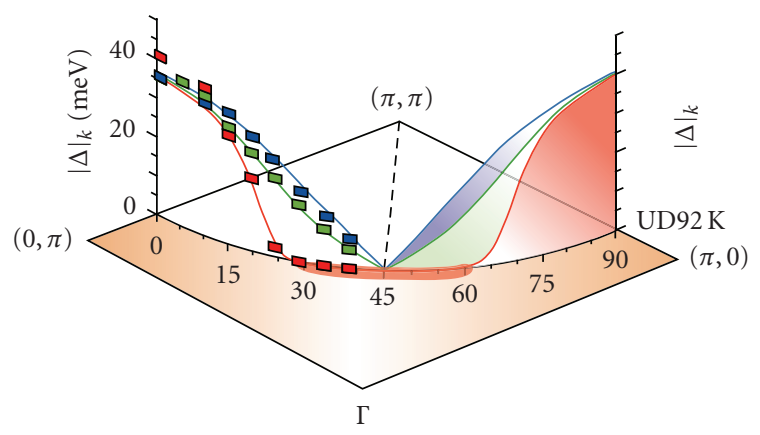

(b)

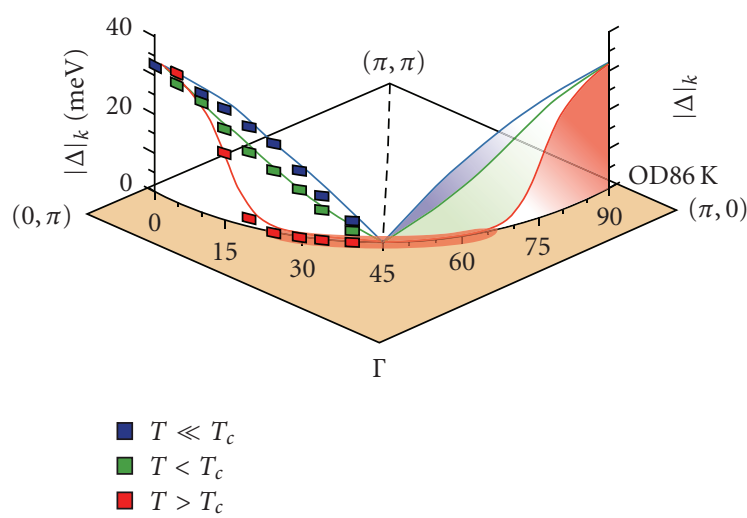

(c)

FIGURE 2: (Color online) Schematic illustrations of the gap function evolution for three different doping levels of $\mathrm{Bi}_{2} \mathrm{Sr}_{2} \mathrm{CaCu}_{2} \mathrm{O}_{8+\delta}$. (a) Underdoped sample with $T_{c}=75 \mathrm{~K}$. (b) Underdoped sample with $T_{c}=92 \mathrm{~K}$. (c) Overdoped sample with $T_{c}=86 \mathrm{~K}$. At $10 \mathrm{~K}$ above $T_{c}$ there exists a gapless Fermi arc region near the node; a pseudogap has already fully developed near the antinodal region (red curves). With increasing doping, this gapless Fermi arc elongates (thick red curve on the Fermi surface), as the pseudogap effect weakens. At $T<$ $T_{c}$ a $d$-wave like superconducting gap begins to open near the nodal region (green curves); however, the gap profile in the antinodal region deviates from the simple $d_{x^{2}-y^{2}}$ form. At a temperature well below $T_{c}\left(T \ll T_{c}\right)$, the superconducting gap with the simple $d_{x^{2}-y^{2}}$ form eventually extends across entire Fermi surface (blue curves) in (b) and (c) but not in (a). (Taken from [403].)

temperature $\left.T_{d}[437,438]\right)$. The interplay between structural and magnetic instabilities is important for pnictides [161], 
since, for example, structural and SDW anomalies appear jointly at $140 \mathrm{~K}$ in $\mathrm{BaFe}_{2} \mathrm{As}_{2}$ [439]. It is not inconceivable that pnictides may be a playground for density waves as well as high- $T_{c}$ oxides, with a rich variety of attendant manifestations.

The DHS is another visiting card of cuprates, being a peculiarity in tunnel and photoemission spectra at low $T \ll T_{c}$ and energies much higher than those of coherent superconducting peaks $[81-83,160,339,347,348,440$, 441]. It is remarkable that in the S-I-N tunnel junctions, where $\mathrm{S}, \mathrm{I}$, and $\mathrm{N}$ stand for a high- $T_{c}$ superconductor, an insulator, and a normal metal, respectively, a DHS might appear for either one bias voltage $V$ polarity only [347] or both $[442,443]$, depending on the specific sample. In SI-N junctions, current-voltage-characteristics (CVCs) with two symmetrically located DHSs (one per branch) are also observed, but with amplitudes that can differ drastically [442, 443]. In S-I-S symmetric junctions, DHS structures are observable (or not) in CVC branches of both polarities simultaneously [347], which seems quite natural. It is very important that although the CVC for every in the series of SI-N junctions with BSCCO as an superconducting electrodes was nonsymmetric, especially due to the presence of the DHS, the CVC obtained by averaging over an ensemble of such junctions turned out almost symmetric, or at least its nonsymmetricity turned out much lower than the nonsymmetricity of every CVC taken into consideration [443].

There is quite a number of interpretations concerning this phenomenon [347, 444-450]. We have discussed most of them in detail in our previous publications, whereas our theory and necessary reference to other models will be presented below.

STM mapping of high- $T_{c}$ oxide samples revealed substantial inhomogeneties of energy gap spatial distribution [334, 336, 338, 339, 341-343, 363, 370, 441, 451-459]. The same conclusion was made from the interlayer tunneling spectroscopy $[460,461]$, more conventional S-I-N tunnel (point-contact) studies $[440,442]$, optical femtosecond relaxation spectroscopy [337], and inelastic neutron scattering measurements [335]. It is quite natural that some inhomogeneity should exist, since the oxygen content is always nonstoichiometric in those compounds [304]. Indeed, correlations were found between oxygen dopant atom positions and the nanoscale electronic disorder probed by STM [336]. The problem has been recently investigated theoretically making allowance for electrostatic modulations of various system parameters by impurity atoms [462].

Nevertheless, the gap distributions occurred to be anomalously large, with sometimes conspicuous two-peak structures in BSCCO [451, 457, 463], $\mathrm{Bi}_{2} \mathrm{Sr}_{1.6} \mathrm{Gd}_{0.4} \mathrm{CuO}_{6+\delta}$ [338], $(\mathrm{Cu}, \mathrm{C}) \mathrm{Ba}_{2} \mathrm{Ca}_{3} \mathrm{Cu}_{4} \mathrm{O}_{12+\delta} \quad$ [440], and $\mathrm{TlBa}_{2} \mathrm{Ca}_{2} \mathrm{Cu}_{2} \mathrm{O}_{10-\delta}$ [442]. Nanoscale electronic nonhomogeneity on the crystal surface was shown to substantially affect the CDW-like DOS modulation observed by STM in $\mathrm{Bi}_{2} \mathrm{Sr}_{1.4} \mathrm{La}_{0.6} \mathrm{CuO}_{6+\delta}$ [340].

Large gap scatterings obviously do not correlate with sharp transitions into the superconducting state at any doping of well prepared samples (implying Cooper-pairing homogeneity), which was demonstrated, for example, by specific heat studies [464]. To solve the problem, one should bear in mind that the gaps measured by STM technique are of two kinds (in our opinion, superconducting gaps and pseudogaps-CDW gaps), which cannot be easily distinguished experimentally [81-83, 160,337]. The guess was proved in [291], where contributions of both gaps in the STM spectra of $\left(\mathrm{Bi}_{0.62} \mathrm{~Pb}_{0.38}\right)_{2} \mathrm{Sr}_{2} \mathrm{CuO}_{6+x}$ were separated by an ingenious trick. Namely, the authors normalized the measured local conductances by removing the larger-gap inhomogeneous background. Then, it became clear that the superconducting gap is more or less homogeneous over the sample's surface, whereas the larger gap (the pseudogap, i.e., the CDW gap) is essentially inhomogeneous.

The intimate origin of the pseudogap variations is currently not understood. At the same time, the inhomogeneity of electron characteristics is also inherent to the related solid solutions BPB, which was demonstrated by spatially resolved electron energy loss spectroscopy [465]. It is reasonable to suggest that this inhomogeneity both in BPB and high- $T_{c}$ oxides is strengthened near free surfaces in agreement with Josephson current measurements across BPB bicrystal tunnel boundaries [466].

Still, there is an interesting phenomenon, which might explain trends for electric properties in cuprates to be inhomogeneous. We mean a spontaneous phase separation, suggested long ago for antiferromagnets [467-470] and the electron gas in paramagnets [471-474]. This idea was later transformed into stripe activity in cuprate and manganite physics, where alternating conducting and magnetic regions constituted separated "phases" [12, 302, 475-480]. Recently, a lot of evidence for local lattice distortions, Jahn-Teller polaron occurrence, and other percolation and filamentary structure formation appeared [177, 217, 228, 481-485], supporting new sophisticated theoretical efforts in the science of phase separation [84, 230, 379, 486-493], mostly but not necessarily dealing with high- $T_{c}$ oxides. The electronic inhomogeneity in cuprates, as discussed above, belongs to the same category of phenomena. Whatever its origin, intrinsic inhomogeneity of cuprates and other oxides seems to be an important feature that needs explanation in order to understand superconductivity (much more homogeneous) itself. Note that electronic phase separation into magnetic and nonmagnetic domains was also found in the iron pnictide superconductor $\mathrm{Ba}_{1-x} \mathrm{~K}_{x} \mathrm{Fe}_{2} \mathrm{As}_{2}$ [494], whereas disorderinduced inhomogeneities of superconducting properties was observed in TiN films [495].

Another high- $T_{c}$ oxide, $\mathrm{YBa}_{2} \mathrm{Cu}_{3} \mathrm{O}_{6+x}$, containing $\mathrm{CuO}$ chains in addition to $\mathrm{CuO}_{2}$ planes, was known for a long time as a material exhibiting one-dimensional CDWs [496]. However, the authors of more recent tunnel measurements [497] concluded that the would-be CDW manifestations might have a different nature, since the observed onedimensional modulation wavelengths have rather a strong dispersion. Nevertheless, it seems that in view of the large CDW amplitude scatter in BSCCO discovered later, this conclusion is premature, with local variations of the FS shape being a possible origin of $\mathrm{CDW}$ wave vector modifications. 
As one sees from the evidence discussed above, CDW modulations are observed in cuprates both directly (as patterns of localized energy-independent electron states in the conventional $\mathbf{r}$-space) and indirectly (as concomitant gapping phenomena). The pseudogap energy $E_{\mathrm{PG}}>\Delta_{\mathrm{SC}}$ constitutes an appropriate scale for CDW gapping. Here, $\Delta_{\mathrm{SC}}$ is the superconducting gap. On the other hand, at low energies $E<\Delta_{\text {SC }}$, single-particle tunneling spectroscopy probes mixed electron-hole $d$-wave Bogoliubov quasiparticles [498], which are delocalized excitations. In this case, it is natural to describe the tunnel conductance in the momentum, k-space. The interference between Bogoliubov quasiparticles is especially strong for certain wave vectors $\mathbf{q}_{i}(i=1, \ldots, 16)$ connecting extreme points on the constant energy contours [499-502]. The interference $\mathbf{k}$-space patterns involve those wave vectors [343, 416, 499, 503-505], this picture being distinct from and complementary to the partially disordered CDW unidirectional or checkerboard structures [344, 359, 365, 371, 458, 506-508].

It is remarkable that interference $\mathbf{r}$-space patterns on cuprate surfaces, the latter being in the superconducting state, are not detected, contrary to the clear-cut STM observations of electron de Broglie standing waves, induced by point defects or step edges, revealed in conductance maps on the normal metal surfaces $[509,510]$. The latter waves are in effect Friedel oscillations [511] formed by twodimensional normal electron density crests and troughs with the wave length $\pi / \mathbf{k}_{F}, \mathbf{k}_{F}$ being the Fermi wave vector. On the other hand, spatial oscillating structures of local DOS in the $d$-wave superconducting state are determined by other representative vectors $\mathbf{q}_{i}$, so that the characteristic oscillations can be denominated as Friedel-like ones at most [502, 512]. Nevertheless, the attenuation of both kinds of spatial oscillations due to superconducting modifications of the screening medium should be more or less similar. Namely, in the isotropic superconducting state, the electron gas polarization operator loses its original singularity at $k=$ $2 k_{F}$ for gapped FS sections [513]. As a consequence, Friedel oscillations gain an extra factor $\exp \left(-2 r / \pi \xi_{0}\right)[514,515]$, where $\xi_{0}$ is the BCS coherence length [498]. For $d$-wave superconductors, the attenuation will be weaker and will totally disappear in the order-parameter node directions. However, those distinctions are not crucial, since the nodes have a zero measure. The modification of screening by formation of Bogoliubov quasiparticles in $d$-wave high- $T_{c}$ oxides explains the absence of conspicuous spatial structures in STM maps, which correspond to the wave vectors $\mathbf{q}_{i}$ mentioned above.

We consider the observed CDWs in oxides as a consequence of electron-hole (dielectric) pairing on the nested sections of corresponding FSs [158-160, 516]. Such a viewpoint is also clearly supported by the experiments in layered dichalcogenides [374-376], the materials analogous to cuprates in the sense of superconductivity appearance against the dielectric (CDW) partial gapping background $[378,379]$. At the same time, other sources of CDW instabilities are also possible $[517,518]$. As for the microscopic mechanism causing CDW formation, it might be an electron-phonon (Peierls insulator) $[519,520]$ or a Coulomb one (excitonic insulator) $[431,521,522]$, or their specific combination. Excitonic instability may also lead to the SDW state $[522,523]$, also competing with superconductivity for the FS [160, 524-529]. It should be noted that researchers asserted that they found plenty of Peierls insulators or partially gapped Peierls metals [158-160, 530-532]. At the same time, the excitonic phase, being mathematically identical in the mean-field limit [533] and physically similar [534] to the Peierls insulator, was not identified unequivocally. One can only mention that some materials claimed to be excitonic insulators, namely, a layered transition-metal dichalcogenide $1 \mathrm{~T}-\mathrm{TiSe}_{2}$ with a commensurate CDW [535, 536], alloys $\mathrm{TmSe}_{0.45} \mathrm{Te}_{0.55}$ [537], $\mathrm{Sm}_{1-x} \mathrm{La}_{x} \mathrm{~S}$ [538], and $\mathrm{Ta}_{2} \mathrm{NiSe}_{5}$ with a direct band gap at the Brillouin zone $\Gamma$ point in the parent high- $T$ state [539]. Therefore it is reasonable that precisely in the later case, the low- $T$ excitonic state is not accompanied by CDWs.

It is necessary to indicate that in many cases, the claimed "charge stripe order" and the more unpretentious "charge order" are an euphemism describing the old good CDWs: "Stripes is a term that is used to describe unidirectional density-wave states, which can involve unidirectional charge modulations (charge stripes) or coexisting charge and spindensity order spin stripes" [12]. We do not think it makes sense to use the term "stripes" in the cases of pure CDW or spin-density-wave (SDW) ordered states. At the same time, this term should be reserved for different possible more general kinds of microseparation [12, 477, 479, 540542], having nothing or little to do with periodic lattice distortions, FS nesting, or Van Hove singularities. The need to avoid misnomers and duplications while naming concepts is quite general in science, as was explicitly stressed by John Archibald Wheeler, who himself coined many terms in physics ("black hole" included) [543].

In this connection, it seems that some experimentalists unnecessarily vaguely attribute the spatially periodical charge structure in the low-temperature tetragonal phase of $\mathrm{La}_{1.875} \mathrm{Ba}_{0.125} \mathrm{CuO}_{4}$, revealed by X-ray scattering [544], to the hypothetical nematic structure or the checkerboard Wigner crystal. Indeed, quite similar spatial charge structures found in $\mathrm{La}_{1.875} \mathrm{Ba}_{0.125-x} \mathrm{Sr}_{x} \mathrm{CuO}_{4}$ by neutron scattering [545] were correctly and without reservation identified as CDWrelated ones, whereas a checkerboard structure (if any) can be considered as a superposition of two mutually perpendicular CDWs. The same can be written about the "stripe" terminology used in [546], where X-ray scattering revealed a periodical charge structure in the low-temperature tetragonal phase of another cuprate $\mathrm{La}_{1.8-x} \mathrm{Eu}_{0.2} \mathrm{Sr}_{x} \mathrm{CuO}_{4}$.

One should mention two other possible collective states competing with Cooper pairing. Namely, these are states with microscopic orbital and spin currents that circulate in the ground state of excitonic insulator (there can be four types of the latter [522]). The concept of the state with current circulation, preserving initial crystal lattice translational symmetry, was invoked to explain cuprate properties [547]. Another order parameter, hidden from clear-cut identification by its supposed extreme sensitivity to sample imperfection, is the so-called $d$-density wave-order parameter $[548,549]$. It is nothing but a CDW order parameter times the same 
form-factor $f(k)=\cos \left(k_{x}\right)-\cos \left(k_{y}\right)$, the product being similar to that for $d_{x^{2}-y^{2}}$-superconductors. Here, $k_{x}$ and $k_{y}$ are the wave-vector components in the $\mathrm{CuO}_{2}$ plane. To some extent, the dielectric order parameter of the BilbroMcMillan model $[159,160,385]$ and its generalizationsthey are presented below-contains the same physical idea as in the $d$-density-wave model: nonuniformity of the CDW gap function in the momentum space.

Although the destructive CDW action on superconductivity of many good materials is beyond question $[56,160$, $380,384,545,550,551]$, it does not mean that maximal $T_{c}$ are limited by this factor only. For instance, $T_{c}$ falls rapidly with the hole concentration $p$ in overdoping regions of $T_{c}-p$ phase diagrams for different $\mathrm{Pb}$-substituted $\mathrm{Bi}_{2} \mathrm{Sr}_{2} \mathrm{CuO}_{6+\delta}$ compounds, even in the case when the critical doping value $p_{\text {cr }}$ corresponding to $T_{d} \rightarrow 0$ lies outside the superconducting dome [552]. A Cu-doped superconducting chalcogenide $\mathrm{Cu}_{x} \mathrm{TiSe}_{2}$ constitutes another example confirming the same trend [380]. Namely, CDW manifestations die out for $x \gtrsim$ 0.06 , whereas $T_{c}$ starts to decrease for $x>x_{\text {optimal }}=0.08$. As has been already mentioned, overdoping can reduce $T_{c}$ simply owing to screening of matrix elements for electronphonon interaction $[99,215,216]$.

\section{Theory of CDW Superconductors and Its Application to Cuprates}

The majority of our results presented below were obtained for $s$-wave superconductors with CDWs. It is a case, directly applicable to many materials (e.g., dichalcogenides, trichalcogenides, tungsten brozes, etc.). On the other hand, as was indicated above, the exact symmetry of the superconducting order parameter in cuprates is not known, although the $d$-wave variant is considered by most researchers in the field as the ultimate truth. Notwithstanding any future solution of the problem, our theory of CDW-related peculiarities in quasiparticle tunnel CVCs can be applied to cuprates, since we are not interested in small energies $e V<\Delta$, where the behavior of a reconstructed DOS substantially depends on whether it is the $s$ - or $d$-wave order parameter [553555]. Here, $e>0$ is the elementary charge, and $\Delta$ is the amplitude of the superconducting order parameter. As for the thermodynamics of CDW superconductors, we present both $s$ - and $d$-cases, each of them having their own specific features.

4.1. Thermodynamics of s-Wave CDW Superconductors. The Dyson-Gorkov equations for the normal $\left(g_{i j}\right)$ and anomalous $\left(\mathcal{F}_{i j}\right)$ temperature Green's functions in the case of coupled superconducting $\Delta_{i j}^{\alpha \gamma}$ and dielectric (CDW) $\Sigma_{i j}^{\alpha \gamma}$ matrix order parameters are the starting point of calculations and can be found elsewhere [160, 386, 397, 426, 427]. Greek superscripts correspond to electron spin projections, and italic subscripts describe the natural split of the FS into degenerate (nested, $d$ ) and non-degenerate (non-nested, $n$ ) sections. For the quasiparticles on the nested sections, the standard condition leading to the CDW gapping holds:

$$
\xi_{1}(\mathbf{p})=-\xi_{2}(\mathbf{p}+\mathbf{Q}),
$$

where $\mathbf{p}$ is the quasimomentum, $\mathbf{Q}$ is the $\mathrm{CDW}$ vector (see the discussion above), Planck's constant $\hbar=1$. This equation binds the electron and hole bands $\xi_{1,2}(\mathbf{p})$ for the excitonic insulator $[431,522]$ and different parts of the one-dimensional self-congruent band in the Peierls insulator case [516]. At the same time, the rest of the FS remains undistorted below $T_{d}$ and is described by the electron spectrum branch $\xi_{3}(\mathbf{p})$. Such an approach was suggested long ago by Bilbro and McMillan [385]. We adopt the strongmixing approximation for states from different FS sections. This means an appearance of a single superconducting order parameter for $d$ and $n d$ FS sections. The spin-singlet structure ( $s$-wave superconductivity and CDWs) of the matrix normal $\left(\Sigma_{i j}^{\alpha \gamma}=\Sigma \delta_{\alpha \beta}\right)$ and anomalous $\left(\Delta_{i j}^{\alpha \gamma}=I_{\alpha \beta}\right)$ self-energy parts (where $\left(I_{\alpha \beta}\right)^{2}=-\delta_{\alpha \beta}$ ) in the weak-coupling limit is suggested. Here, $\delta_{\alpha \beta}$ is the Kronecker delta. The selfconsistency equations for the order parameters obtained in accordance with the fundamentals can be expressed in the following form [386]:

$$
\begin{aligned}
& 1=V_{\mathrm{BCS}} N(0)[\mu I(D)+(1-\mu) I(\Delta)], \\
& 1=V_{\mathrm{CDW}} N(0) \mu I(D),
\end{aligned}
$$

where

$$
I(x)=\int_{0}^{\Omega} \frac{d \xi}{\sqrt{\xi^{2}+x^{2}}} \tanh \frac{\sqrt{\xi^{2}+x^{2}}}{2 T} .
$$

Here, the Boltzmann constant $k_{\mathrm{B}}=1, V_{\mathrm{BCS}}$ and $V_{\mathrm{CDW}}$ are contact four-fermion interactions responsible for superconductivity and CDW gapping, respectively. The gap

$$
D(T)=\left[\Delta^{2}(T)+\Sigma^{2}(T)\right]^{1 / 2}
$$

is a combined gap appearing on the nested FS sections, whereas the order parameter $\Delta$ defines the resulting observed gap on the rest of the FS (compared with the situation in cuprates $[344,350,356,403])$. The parameter $\mu$ characterizes the degree of the FS dielectrization (hereafter, we use this nonconventional term instead of "gapping" in some places to avoid confusion with the superconducting gapping), so that $N_{d}(0)=\mu N(0)$ and $N_{n d}(0)=(1-\mu) N(0)$ are the electron DOSs per spin on the FS for the nested and nonnested sections, respectively. The upper limit in (3) is the relevant cut-off frequency, which is assumed to be equal for both interactions. If the cut-offs BCS and CDW are considered different, the arising correction, $\log \left(\Omega_{\mathrm{CDW}} / \Omega_{\mathrm{BCS}}\right)$, is logarithmically small [385] and does not change qualitatively the subsequent results. Only in the case of almost complete electron spectrum dielectric gapping $(\mu \rightarrow 1)$ does the difference between BCS and CDW become important for the phase coexistence problem [433]. This situation is, however, of no relevance for substances with detectable superconductivity, since $T_{c}$ tends to zero for $\mu \rightarrow 1$. In this subsection, we confine ourselves to the case $\operatorname{Re} \Sigma>0$, $\operatorname{Im} \Sigma=0$, since the phase $\varphi$ of the complex order parameter $\Sigma \equiv|\Sigma| e^{i \varphi}$ does not affect the thermodynamic properties, whereas tunnel currents do depend on $\varphi[160,556,557]$, which will be demonstrated explicitly below. 
Introducing the bare order parameters $\Delta_{0}=$ $2 \Omega \exp \left[-1 / V_{\mathrm{BCS}} N(0)\right]$ and $\Sigma_{0}=2 \Omega \exp \left[-1 / V_{\mathrm{CDW}} N_{d}(0)\right]$, we can rewrite the system of (2) in an equivalent form, convenient for numerical calculations:

$$
\begin{gathered}
I_{M}[\Delta, T, \Delta(0)]=0, \\
I_{M}\left(D, T, \Sigma_{0}\right)=0,
\end{gathered}
$$

where

$I_{M}\left(G, T, G_{0}\right)=\int_{0}^{\infty}\left(\frac{1}{\sqrt{\xi^{2}+G^{2}}} \tanh \frac{\sqrt{\xi^{2}+G^{2}}}{2 T}-\frac{1}{\sqrt{\xi^{2}+G_{0}^{2}}}\right) d \xi$

is the standard Mühlschlegel integral [558], the root of which $G=s \mathrm{Mu}\left(G_{0}, T\right)$ is the well-known gap dependence for the $s$-wave BCS superconductor [9], $G_{0}=G(T=0)$, and [385]

$$
\Delta(0)=\left(\Delta_{0} \Sigma_{0}^{-\mu}\right)^{1 /(1-\mu)} .
$$

However, (5) mean that both gaps $\Delta(T)$ and $D(T)$ have the BCS form $G=s \mathrm{Mu}\left(G_{0}, T\right)$ [386], namely: (i) $\Delta(T)=$ $s \mathrm{Mu}[\Delta(0), T]$, that is, the actual value of the superconducting gap of the CDW superconductor at $T=0$ is $\Delta(0)$ rather than $\Delta_{0}$, and the actual superconducting critical temperature is $T_{c}=\gamma \Delta(0) / \pi$; (ii) at the same time, $D(T)=s \mathrm{Mu}\left(\Sigma_{0}, T\right)$, which determines $T_{d}=\gamma \Sigma_{0} / \pi$. Here, $\gamma=1.7810 \ldots$ is the Euler constant.

From (4), we obtain that, at $T=0$,

$$
\Sigma_{0}^{2}=\Delta^{2}(0)+\Sigma^{2}(0) .
$$

Replacing $\Delta(0)$ by its value (7), we arrive at the conclusion that in the model of $s$-wave superconductor with partial CDW gapping, two order parameters coexist only if $\Delta_{0}<\Sigma_{0}$. Then, according to (7), $\Delta(0)<\Delta_{0}$; that is, the formation of the CDW, if it happens, always inhibits superconductivity, in agreement with the totality of experiments $[160,375,380$, 382, 551]. Also, vice versa, according to $(4)$, for $T<T_{c}$, $\Sigma(T)<s \mathrm{Mü}\left(\Sigma_{0}, T\right)$; that is, superconductivity suppresses dielectrization.

In Figure 3, the dependences $\Delta(T)$ and $\Sigma(T)$ are shown for various parameters of the partially dielectrized CDW $s$-wave superconductor. It can be easily inferred from the data shown in both panels that, in agreement with the foregoing, $\Delta(T) / \Delta(0)$ curves coincide with the Mühlschlegel one for any values of the dimensionless parameters $\mu$ and $\sigma_{0} \equiv \Sigma_{0} / \Delta_{0}$. The novel feature, which has been overlooked in other investigations, is the possibility of such a strong suppression of $\Sigma$ for low enough $T$ that it becomes smaller than $\Delta$, although $T_{d}$ is larger than $T_{c}$ (see Figure 3(b)). This intriguing situation can be realized for the parameter $\sigma_{0}$ close to unity. One should note that the actual gaps $\Delta$ and $D$ (the former coincides with the superconducting order parameter) are monotonic functions of $T$. However the dielectric order parameter is not.

The magnitudes of $T_{c}$ and $\Delta(0)$ strongly depend on $\mu$ and $\sigma_{0}$, although the simple BCS-like scaling between them survives, that is, for CDW $s$-wave superconductors $\Delta(0) / T_{c}=$ $\pi / \gamma \approx 1.76$. Although for, say, $\Sigma_{0} \geq 1.5 \Delta_{0}$ and reasonable $\mu=0.5$ [386], the demand of self-consistency between $\Sigma(T)$ and $\Delta(T)$ becomes less important quantitatively. It justifies our previous approach with $T$-independent $\Sigma$ [427] and the estimation of combined gap as $\left(\Delta_{\mathrm{BCS}}^{2}(T)+\Delta_{\mathrm{PG}}^{2}\right)^{1 / 2}$ with $T$ independent $\Delta_{\mathrm{PG}}$ made on the basis of interlayer tunneling measurements in BSCCO mesas [559]; self-consistency leads to new qualitative effects and cannot be avoided. As for the magnitude of the very $\Delta_{\mathrm{PG}}$, inferred from tunneling measurements, it was found in [559] to be substantially smaller than that of $\Delta_{\mathrm{BCS}}(T \rightarrow 0)$, whereas the opposite case turned out to be true both for BSCCO [349, 399, 560, 561], $\mathrm{Bi}_{2-x} \mathrm{~Pb}_{x} \mathrm{Sr}_{2} \mathrm{CaCu}_{2} \mathrm{O}_{8+\delta}$ [460], and $(\mathrm{Bi}, \mathrm{Pb})_{2} \mathrm{Sr}_{2} \mathrm{Ca}_{2} \mathrm{Cu}_{3} \mathrm{O}_{10+\delta}$ [562]. Other tunnel measurement for BSCCO [417] revealed $\Delta_{\mathrm{PG}}>\Delta_{\mathrm{BCS}}(T \rightarrow 0)$ for underdoped samples and $\Delta_{\mathrm{PG}}<$ $\Delta_{\mathrm{BCS}}(T \rightarrow 0)$ for overdoped ones. A marked sensitivity of $\Delta_{\mathrm{PG}}$ to doping together with strong inhomogeneity, discovered in Bi-based ceramics [334-336, 338, 343, 359, 440, 441, 456-458, 563, 564] and $\mathrm{Ca}_{2-x} \mathrm{Na}_{x} \mathrm{CuO}_{2} \mathrm{Cl}_{2}$ [565], may be responsible for the indicated discrepancies.

Since the BCS character of the gap dependences for the CDW $s$-wave superconductor is preserved, the $T$-dependence of the heat capacity $C$ for the doubly gapped electron liquid (i.e., below the actual $T_{c}$ ) equals to the superposition of two BCS-like functions:

$$
C(T)=\frac{2 \pi^{2} N(0)}{3}\left[(1-\mu) T_{c} c_{\mathrm{BCS}}\left(\frac{T}{T_{c}}\right)+\mu T_{d} c_{\mathrm{BCS}}\left(\frac{T}{T_{d}}\right)\right],
$$

where

$$
c_{\mathrm{BCS}}\left(t=\frac{T}{T_{c}^{\mathrm{BCS}}}\right)=\frac{C_{\mathrm{BCS}}(T)}{C_{\mathrm{BCS}}\left(T=T_{c}^{\mathrm{BCS}}+0\right)} .
$$

It should be noted that the normalized discontinuity $\Delta C / C_{n}\left(T_{c}\right)$ at the superconducting phase transition may also serve as indirect evidence for the CDW gap on the FS, because in this case it is not at all a trivial BCS jump:

$$
\frac{\Delta C_{\mathrm{BCS}}}{\gamma_{S} T_{c}}=\frac{12}{7 \zeta(3)} \approx 1.43 .
$$

Here, $C_{n}(T)=\gamma_{S} T \equiv\left(2 \pi^{2} N(0) / 3\right) T$ is the normal electron-gas heat capacity, whereas $\gamma_{S}$ is the Sommerfeld constant. CDW-driven deviations from the BCS behavior was recognized long ago $[425,566]$. However, only the selfconsistent approach [386] allows us to give a quantitative answer at any value of the parameters appropriate to the partially CDW-gapped superconductor. It can be seen from Figure 4(a), where the conventionally normalized superconducting phase transition anomaly is shown as a function of $\mu$. The discontinuity is always smaller than the BCS value (11), in agreement with previous qualitative considerations $[425,566]$. At the same time, the BCS ratio is restored not only for $\mu=0$, that is, in the absence of the dielectrization, but also for $\mu \rightarrow 1$. In the former case, it is clear, because we are dealing with a conventional BCS superconductor. On the other hand, for large enough $\mu$, CDW gapping 


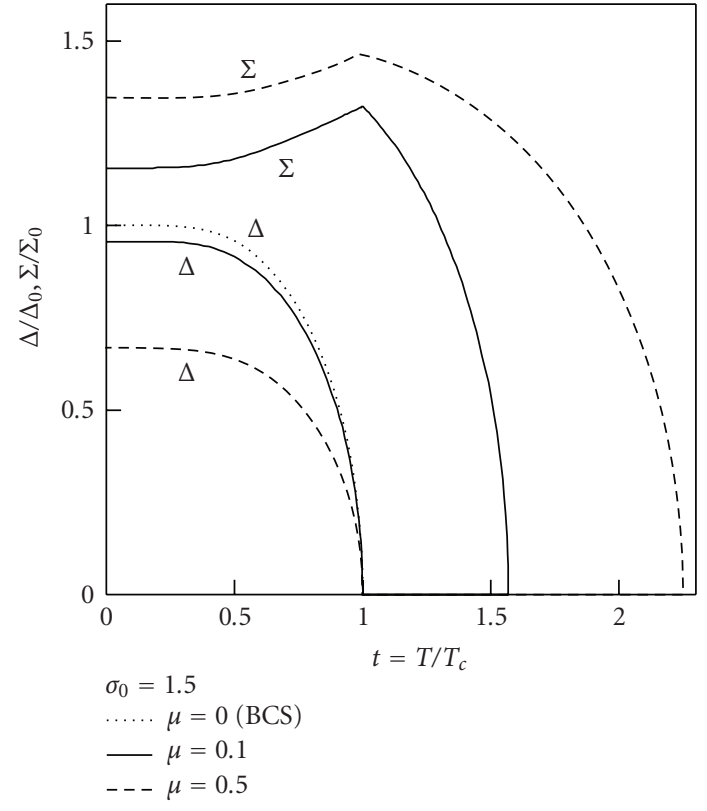

(a)

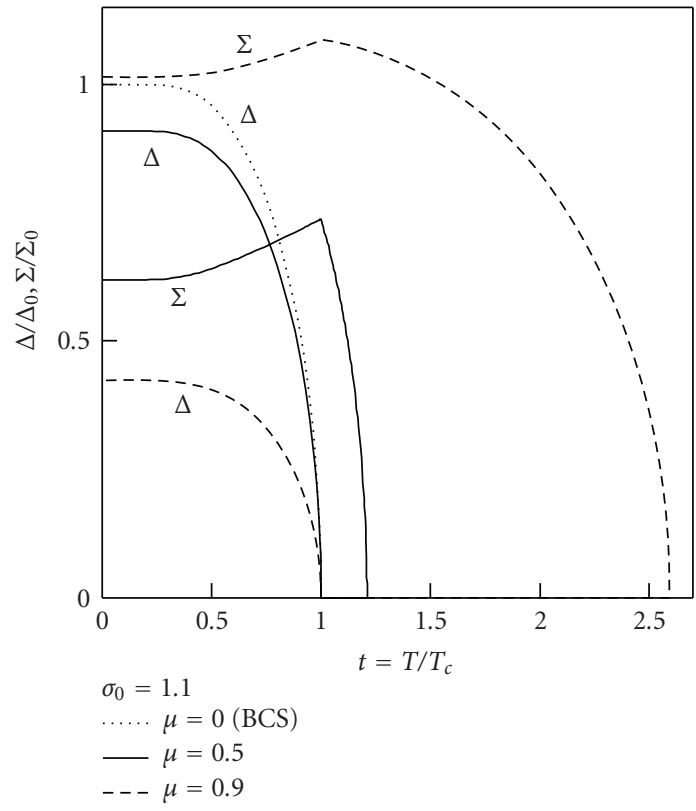

(b)

FIgURE 3: Temperature dependences of the superconducting $(\Delta)$ and dielectric $(\Sigma)$ order parameters for different values of the dimensionless parameters $\mu$ (the portion of the nested Fermi surface sections, where the charge-density-wave, CDW, gap develops) and $\sigma_{0}$ (see explanations in the text). (Taken from [386].)

almost completely destroys superconductivity, so $T_{c} \ll T_{d}$. Therefore, in the relevant superconducting $T$ range, the contribution to $C(T)$ from the $d$ FS sections, governed by the gap $D \approx \Sigma$, becomes exponentially small. Another term, determined by the $n$ FS section, ensures the BCS limiting value of the normalized discontinuity.

The dependences of $\Delta C / C_{n}$ on $\sigma_{0}$ for various values of $\mu$ are depicted in Figure 4(b). One sees that the effect is large for $\sigma_{0}$ close to unity, whereas the difference between 1.43 and $\Delta C / C_{n}$ goes to zero as $\sigma_{0}^{-2}$, verifying the asymptotical result [425]. It should be noted that the heat capacity calculation scheme adopted for $s$-wave CDW superconductors can be applied also to other types of order parameter symmetry.

Experimental data on heat capacity, which could confirm the expressed ideas, are scarce. For $\mathrm{Nb}_{3} \mathrm{Sn}$, it was recently shown by specific heat measurements using the thermal relaxation technique that $T_{c} \approx 17 \div 18 \mathrm{~K}$ is reduced when the critical temperature of the martensitic transition $T_{d} \approx$ $42 \div 53 \mathrm{~K}$ grows [567]. Unfortunately, a large difference between $T_{c}$ and $T_{d}$ made the effects predicted by us quite small here, which is probably the reason why they have not been observed in these studies.

As for cuprates, reference should be made to $\mathrm{La}_{2-x} \mathrm{Ba}_{x} \mathrm{CuO}_{4-y}$ [568], $\mathrm{La}_{2-x} \mathrm{Sr}_{x} \mathrm{CuO}_{4-y}$ [569], and $\mathrm{YBa}_{2} \mathrm{Cu}_{3} \mathrm{O}_{7-y}$ [570], where underdoping led to a reduction of $\Delta C / C_{n}$. The same is true for measurements of the heat capacity in $\mathrm{Bi}_{2} \mathrm{Sr}_{2-x} \mathrm{La}_{x} \mathrm{CuO}_{6+\delta}$ single crystals [571], which demonstrated that the ratio $\Delta C / C_{n}$ for a strongly underdoped sample turned out to be about 0.25 , that is, much below BCS values $12 / 7 \zeta(3) \approx 1.43$ and $8 / 7 \zeta(3) \approx 0.95$ [572] for $s$-wave and $d$-wave superconductivity, respectively.
There is also an opposite evidence for the relationship $\Delta C / C_{n}>1.43$, for example, in the electron-doped high- $T_{c}$ oxide $\operatorname{Pr}_{1.85} \mathrm{Ce}_{0.15} \mathrm{CuO}_{4-\delta}$ [573]. More details, as well as information on other CDW superconductors, can be found in [386]. In any case, despite the well-known challenging controversy for BPB $[392,574-576]$, the problem was not studied enough for any superconducting oxide family, probably due to experimental difficulties.

4.2. Enhancement of the Paramagnetic Limit in s-Wave CDW Superconductors. Upper critical magnetic fields $H_{c 2}$ [577579] (along with critical currents [132, 579, 580]) belong to main characteristics of superconductors crucial for their applications. In particular, knowing the upper limits on upper critical fields is necessary to produce superconducting materials for high-performance magnets, not to talk about scientific curiosity.

One of such limiting factors is the paramagnetic destruction of spin-singlet superconductivity, which was discovered long ago theoretically by Clogston [581] and Chandrasekhar [582]. In the framework of the BCS theory, they obtained a limit

$$
H_{p}^{\mathrm{BCS}}=\frac{\Delta_{\mathrm{BCS}}(T=0)}{\mu_{\mathrm{B}}^{*} \sqrt{2}}
$$

from above on $H_{c 2}$ at zero temperature, $T$. Here, $\Delta_{B C S}(T)$ is the energy gap in the quasiparticle spectrum of BCS $s$ wave superconductor, and $\mu_{\mathrm{B}}^{*}$ is the effective Bohr magneton, which may not coincide with its bare value $\mu_{\mathrm{B}}=e \hbar / 2 m c$, where $\hbar$ is Planck's constant, equal to unity in the whole 


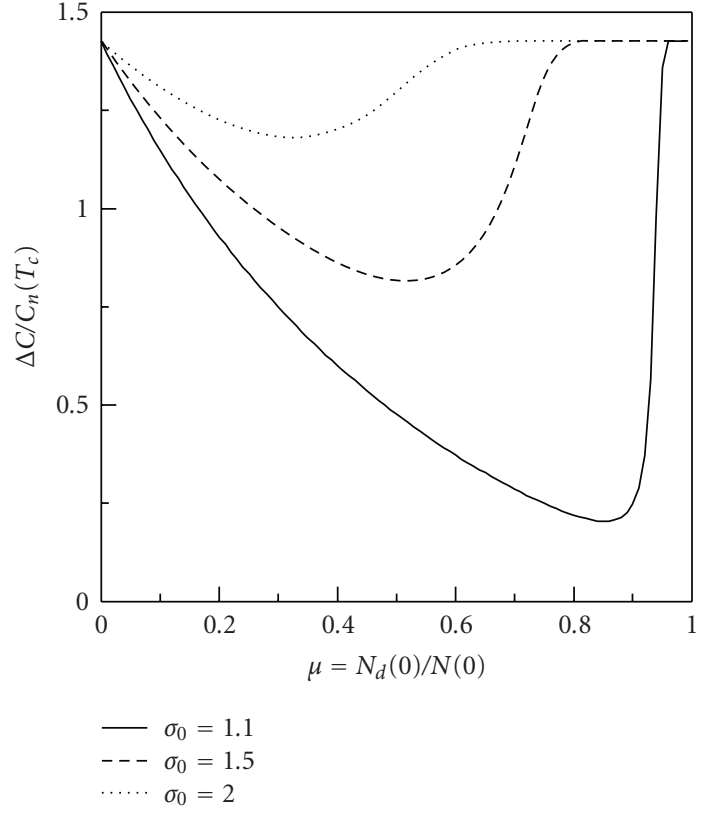

(a)

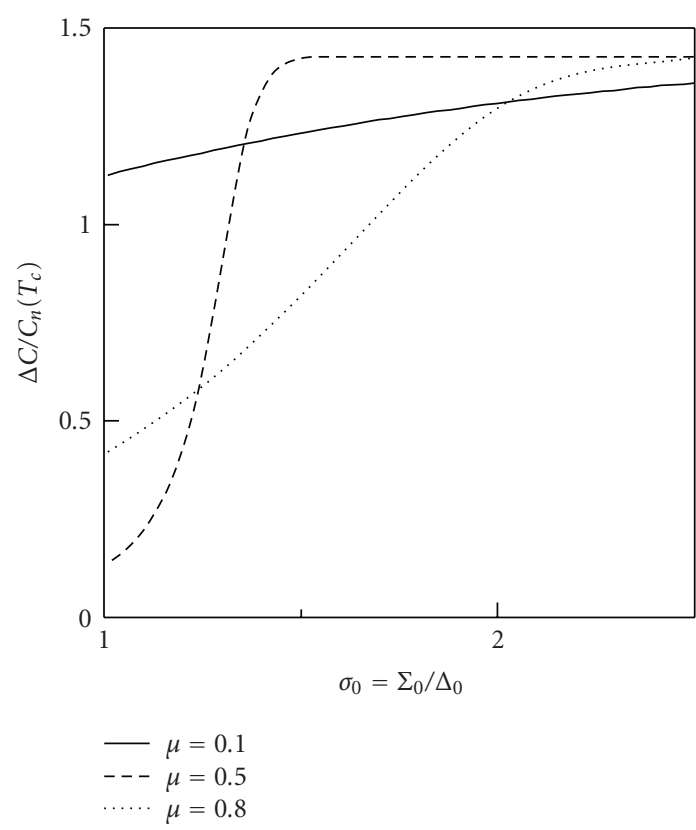

(b)

Figure 4: Dependences of the normalized heat capacity discontinuity $\Delta C$ at $T_{c}$ on $\mu$ (a) and $\sigma_{0}$ (b). (Taken from [386].)

article but shown here explicitly for clarity, $m$ is the electron mass, and $c$ is the velocity of light.

Limit (12) may be overcome at a high concentration of strong spin-orbit scatterers, when the spins of the electrons, constituting the Cooper pairs, are flipped [583]. Then, the actual $H_{c 2}(T=0)$ starts to exceed [584] the classical bound. Such an enhancement of $H_{c 2}$ has been observed, for example, in $\mathrm{Al}$ films coated with Pt monolayers [585]. The Pt atoms served there as strong spin-orbit scatterers due to their large nuclear charge $Z$. One should indicate a possibility of exceeding value (12), if the energy, $E$, dependence of the normal state density of states is significant, which is the case in the neighborhood of the van Hove singularity [518]. Then, the BCS approximation of $N(E) \simeq N(0)$ is no longer valid, so that the actual $H_{p}$ may become larger than limit (12) [586].

We have found another reason, why the ClogstonChandrasekhar value can be exceeded. Namely, it is the appearance of a partial CDW-driven dielectric gap on the $d$ sections of the FS [427, 587-589]. The expected increase of the calculated limiting paramagnetic field $H_{p}$ for CDW superconductors, as compared to $H_{p}^{\mathrm{BCS}}$, is intimately associated with paramagnetic properties of the normal CDW state, which are very similar to those for BCS $s$-wave superconductors [382, 590-592].

It should be emphasized that the very self-consistency of the two-gap solution [386] made the treatment of the paramagnetic limit problem [589] transparent and less involved than previous approximations $[427,587,588]$.

To calculate the paramagnetic limit, we considered the relevant free energies $F$ per unit volume for all possible ground state phases in an external magnetic field $H$. The parent nonreconstructed phase (actually existing only above $T_{d}$ !), with both superconducting and CDW pairings switched off and in the absence of $H$, served as a reference point. At $T<T_{d}$, we deal with relatively small differences $\delta F$ reckoned from this hypothetical "doubly-normal" state [498]. In our case, in the Clogston-Chandrasekhar spirit [581, 582], there are two energy differences to be compared [589], specifically, that of a paramagnetic phase in the magnetic field [593] (diamagnetic effects are not taken into account when one is interested in the paramagnetic limit per se)

$$
\delta F_{p}=-N(0)\left(\mu_{\mathrm{B}}^{*} H\right)^{2}
$$

and that of a CDW-superconducting phase

$$
\delta F_{s}=-N_{n}(0) \frac{\Delta^{2}(0)}{2}-N_{d}(0) \frac{D^{2}(0)}{2} .
$$

Here, $\Delta(0)$ is determined by $(7)$, whereas $D(0)$, as stems from (8), is equal to $\Sigma_{0}=\pi T_{d} / \gamma$. A simple algebra leads to the analytical equation for the increase of the paramagnetic limit over the Clogston-Chandrasekhar value (12):

$$
\left(\frac{H_{p}}{H_{p}^{\mathrm{BCS}}}\right)^{2}=1+\mu\left[\left(\frac{\Sigma_{0}}{\Delta_{0}}\right)^{2 /(1-\mu)}-1\right] .
$$

This relationship is expressed in terms of genuine (bare) system parameters $\mu, \Sigma_{0}$, and $\Delta_{0}$. However, experimentalists are interested in the dependence of $H_{p} / H_{p}^{\mathrm{BCS}}$ on actual measurable quantities. The transformation of (15) can be easily made, and one arrives at the final formula

$$
\begin{aligned}
\left(\frac{H_{p}}{H_{p}^{\mathrm{BCS}}}\right)^{2} & =1+\mu\left[\left(\frac{\Sigma(0)}{\Delta(0)}\right)^{2}-1\right] \\
& =1+\mu\left[\left(\frac{T_{d}}{T_{c}}\right)^{2}-1\right] .
\end{aligned}
$$




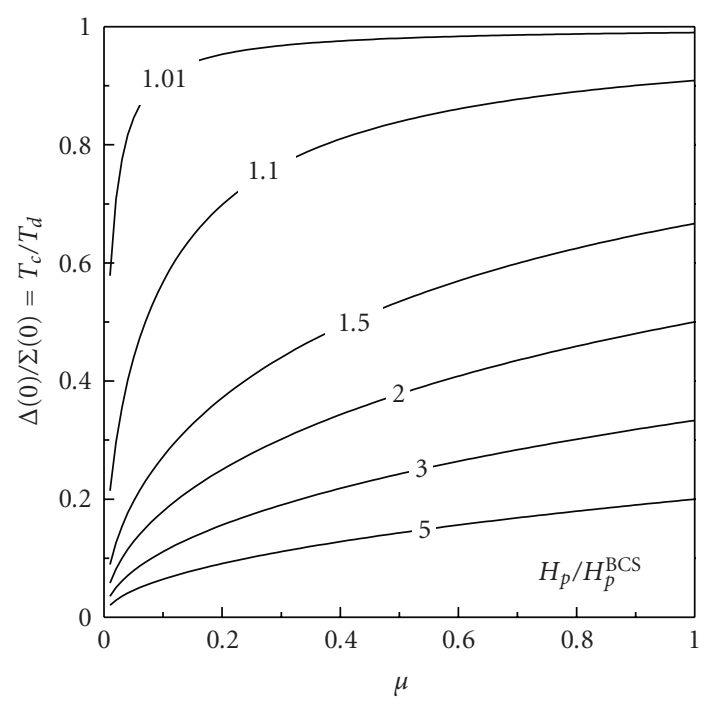

Figure 5: Contour plot of the ratio $H_{p} / H_{p}^{\text {BCS }}$ on the plane $\left(T_{c} / T_{d}, \mu\right)$. Here $H_{p}$ is the paramagnetic limit for CDW superconductors and $H_{p}^{\mathrm{BCS}}$ is that for BCS spin-singlet superconductors, $T_{c}$ and $T_{d}$ are the observed critical temperatures of the superconducting and CDW transitions, respectively. (Taken from [589].)

To calculate the expected paramagnetic limit, one needs to know $\mu$, which was estimated, for example, as 0.2 for $\mathrm{NbSe}_{3}$ [594] or 0.15 for $\mathrm{La}_{2-x} \mathrm{Sr}_{x} \mathrm{CuO}_{4}$ [595].

The contour curves in the parameter space obtained from (16) are displayed in Figure 5 One can readily see that for typical $T_{c} / T_{d} \approx 0.05-0.2$ (some A15 compounds are rare exceptions [160]) and moderate values of $\mu \approx 0.3-0.5$, the augmentation of the paramagnetic limit becomes very large. There is a number of CDW superconductors [589], where the increase of the paramagnetic limit was detected, in accordance with the results presented here. Unfortunately, not so much can be said about high- $T_{c}$ oxides. It seems that extremely high values of $H_{c 2}$ observed in these materials are the reason of the unjustified neglect to the problem.

\subsection{Dip-Hump Structures and Pseudogaps in Tunnel Current-} Voltage Characteristics for Junctions Involving CDW Superconductors. In Section 3, a lot of evidence was presented concerning dip-hump structures (DHSs) and pseudogaps in high- $T_{c}$ oxides $[81-83,160,559]$. Broadly speaking, pseudogaps and DHSs have much in common. In particular, they can coexist with superconducting coherent peaks, their appearance in current-voltage characteristics (CVCs) is to some extent random, and their shapes are sampledependent. Therefore, a suggestion inevitably arises that those two phenomena might be governed by the same mechanism. Our main assumption is that both pseudogaps and dip-hump structures are driven by CDW instabilities discussed above and that their varying appearances are coupled with the intrinsic, randomly inhomogeneous electronic structure of cuprates. In the strict sense, according to the adopted scenario, both DHSs and pseudogaps are the manifestations of the same dielectric DOS depletion, the former being a result of superimposed CDW- and superconductivity-induced CVC features below $T_{c}$. To justify our approach, it is crucial that direct spatial correlations between irregular patterns of CDW three-dimensional supermodulations [365] and topographic maps of the superconducting gap amplitudes on the BSCCO surface were displayed by tunneling spectroscopy [458].

A detailed description of our approaches to the problems of tunneling through junctions with CDW isotropic superconductors as electrodes, and the emergence of DHSs in the CVCs of high- $T_{c}$ oxides can be found elsewhere [81$83,160,386,596]$. Here, we will present only a summary of our new results, briefly touching only those issues that are necessary for the rest of the paper to be self-contained.

We should emphasize different roles of the order parameter phases in determining quasiparticle tunnel currents. Concerning the superconducting order parameter, its phase may be arbitrary unless we are interested in the Josephson current across the junction. On the other hand, the CDW phase $\varphi$ governs quasiparticle CVCs of junctions with CDW superconductors as electrodes $[556,557]$. The value of $\varphi$ can be pinned by various mechanisms in both excitonic and Peierls insulators, so that $\varphi$ acquires the values either 0 or $\pi$ in the first case [522] or is arbitrary in the unpinned state of the Peierls insulator [516]. At the same time, in the case of an inhomogeneous CDW superconductor, which will be discussed below, a situation can be realized, where $\varphi$ values are not correlated over the junction area. Then, the contributions of elementary tunnel currents may compensate one another to some extent, and this configuration can be phenomenologically described by introducing a certain effective phase $\varphi_{\text {eff }}$ of the CDW order parameter. If the spread of the phase $\varphi$ is random, the most probable value for $\varphi_{\text {eff }}$ is $\pi / 2$, and the CVC for a nonsymmetric junction involving CDW superconductor becomes symmetric.

Most often, CVCs for cuprate-I-N (i.e., S-I-N) junctions reveal a DHS only at $V=V_{S}-V_{N}<0$ [597-599], so that the occupied electron states below the Fermi level are probed for CDW superconductors. In our approach, it corresponds to the phase $\varphi$ close to $\pi$. This preference may be associated with some unidentified features of the CDW behavior near the sample surface.

On the other hand, there are S-I-N junctions, where DHS structures are similar for both $V$ polarities [347, 442, $443,600]$. As for those pseudogap features, which were unequivocally observed mostly at high $T$, no preferable $V$ sign of their manifestations was found. We note that the symmetricity of the tunnel conductance $G(V)=d J / d V$, where $J$ is the tunnel current through the junction, might be due either to the microscopic advantage of the CDW state with $\varphi=\pi / 2$ or to the superposition of different current paths in every measurement covering a spot with a linear size of a CDW coherence length at least. Both possibilities should be kept in mind. The variety of $G(V)$ patterns in the S-I-N set-up for the same material and with identical doping is very remarkable, showing that the tunnel current is rather sensitive to the CDW phase $\varphi$. Nevertheless, the very appearance of the superconducting domain structure for cuprates with local domain-dependent gaps and critical 
temperatures [601] seems quite plausible for materials with small coherence lengths. Essentially the same approach has been proposed earlier to explain superconducting properties of magnesium diboride [78].

On the basis of information presented above and using the self-consistent solutions for $\Delta(T)$ and $\Sigma(T)$, we managed to describe the observed rich variety of $G(V)$ patterns by calculating quasiparticle tunnel CVCs $J(V)$ for two typical experimental set-ups. Namely, we considered S-I-N and S-I$S$ junctions, where "S" here means a CDW superconductor. A unique tunnel resistance in the normal state $R$ enters into all equations, since we assume the incoherent tunneling to occur, in accordance with the previous analysis for BSCCO [122, 602]. The used Green's function method followed the classical approach of Larkin and Ovchinnikov [603]. We skip all (quite interesting) technical details, since they can be found elsewhere [81-83, 160, 386, 596].

The obtained equations for $J(V)$ form the basis for calculations both $J(V)$ and $G(V)$ (sub-, superscripts $n s$ and $s$ denote S-I-N and S-I-S junctions, resp.). They must be supplemented with a proper account of the nonhomogeneous background, since, as was several times stressed above, STM maps of the cuprate crystal surfaces consist of random nano-scale patches with different gap depths and widths, as well as coherent edge sharpnesses. In this connection, our theory assumes the combination CDW + inhomogeneity to be responsible for the appearance of the DHSs. Our main conclusion is that it is the dispersion of the parameter $\Sigma_{0}$ and, as a result, the $D$-peak smearing (the $\Delta$-peak also becomes smeared but to a much lesser extent) - that is the most important to reproduce experimental pictures. The value of the FS gapping degree $\mu$ is mainly responsible for the amplitude of the DHSs. At the same time, neither the scattering of the parameter $\mu$ nor that of the superconducting order parameter $\Delta_{0}$ can result in the emergence of smooth DHSs, so that sharp CDW features remain unaltered. Therefore, for our purpose, it was sufficient to average only over $\Sigma_{0}$ rather than simultaneously over all parameters of CDW superconductors, although the variation of any individual parameter made the resulting theoretical CVCs more similar to experimental ones.

Although it is a well-recognized matter of fact that CDWdriven $D$-singularities in $G(V)$ scatter more strongly for a nonhomogeneous medium than main coherent superconducting peaks at $e V= \pm \Delta$ (S-I-N junctions) or $\pm 2 \Delta$ (S-I$\mathrm{S}$ junctions), this phenomenon has not yet been explained. It seems that the sensitivity of the Peierls [604, 605] or excitonic-insulator $[431,606]$ order parameters to the Coulomb potential of the impurities, for example, oxygen ions, might be the reason of such a dispersion. On the other hand, $s$-wave superconductivity is robust against impurity influence (Anderson theorem [607-609]). As for anisotropic superconductivity with $d$-wave or other kinds of symmetry, they are suppressed by nonmagnetic impurity scattering $[3,554,610,611]$ due to scattering-induced order parameter isotropization. Their survival in disordered cuprate samples, especially in the context of the severe damage inflicted by impurities on the pseudogap, testifies that the Cooper-pair order parameter includes a substantial isotropic component.
The parameter $\Sigma_{0}$ was assumed to be distributed within the interval $\left[\Sigma_{0}-\delta \Sigma_{0}, \Sigma_{0}+\delta \Sigma_{0}\right]$. The normalized weight function $W(x)$ was considered as a bell-shaped fourth-order polynomial within this interval and equal to zero beyond it (see the discussions in [81]). In any case, the specific form of $W(x)$ is not crucial for the final results and conclusions.

Our approach is in essence the BCS-like one. It means, in particular, that we do not take a possible quasiparticle "dressing" by impurity scattering and the electron-boson interaction, as well as the feedback influence of the superconducting gapping, into account $[612,613]$. Those effects, important per se, cannot qualitatively change the random two-gap character of superconductivity in cuprates.

As was already mentioned, we have assumed so far that both $\Delta$ and $\Sigma$ are $s$-wave-order parameters. Nevertheless, our approach to CVC calculations is qualitatively applicable to superconductors with the $d$-wave symmetry, if not to consider the intragap voltage range $|e V|<\Delta$.

The results of calculations presented below show that the same CDW + inhomogeneity combination can explain DHSs at low $T$ as well as the pseudogap phenomena at high $T$, when the DHS is smoothed out. Thus, theoretical $T$-dependences of tunnel CVCs mimic the details of the DHS transformation into the pseudogap DOS depletion for nonsymmetric and symmetric junctions, involving cuprate electrodes. We consider the CDW-driven phenomena, DHS included, as the tip of an iceberg, a huge underwater part of which is hidden by strong superconducting manifestations, less influenced by randomness than their CDW counterpart. To uncover this part, one should raise $T$, which is usually done with no reference to the DHS, the latter being substantially smeared by the Fermi-distribution thermal factor. It is this DOS depletion phenomenon that is connected to the pseudogapping phenomena [14, 18, 334, 348, 399, 441].

The results of calculations of $G^{n s}(V)$ in the case when parameter $\Sigma_{0}$ is assumed to scatter are shown in Figure 6 for $\varphi=\pi$. The value $\varphi=\pi$ was selected, because this case corresponds to the availability of the DHS in the negativevoltage branch of the nonsymmetric CVC, and such an arrangement is observed in the majority of experimental data. In accordance with our basic equations, all the four existing CVC peculiarities at $e V= \pm \Delta$ and $\pm D$ become smeared, although to various extent: the large singularities at $e V= \pm \Delta$ almost preserve their shape, the large singularity at $e V=-D$ transforms into a DHS, and the small one at $e V=D$ disappears on the scale selected. The one-polarity dip-hump peculiarity in experimental CVCs for BSCCO [597] is reproduced excellently. Owing to relationship (7), the actual parameter $\Delta$ also disperses, but, due to the small value of $\mu$, this fluctuation becomes too small to be observed in the plot. Thus, the calculated CVCs of Figure 6 demonstrate all principal features intrinsic to the tunnel conductivity of S-I-N junctions at low $T$, involving CDW superconductors, specifically, asymmetry with respect to the $V$ sign is associated with the phase $\varphi \neq \pi / 2$ of the CDWorder parameter, the emerging CDW induces singularities at $e V= \pm D$, whereas the intrinsic CDW inhomogeneity transforms the major one into a DHS, totally suppressing the minor. 


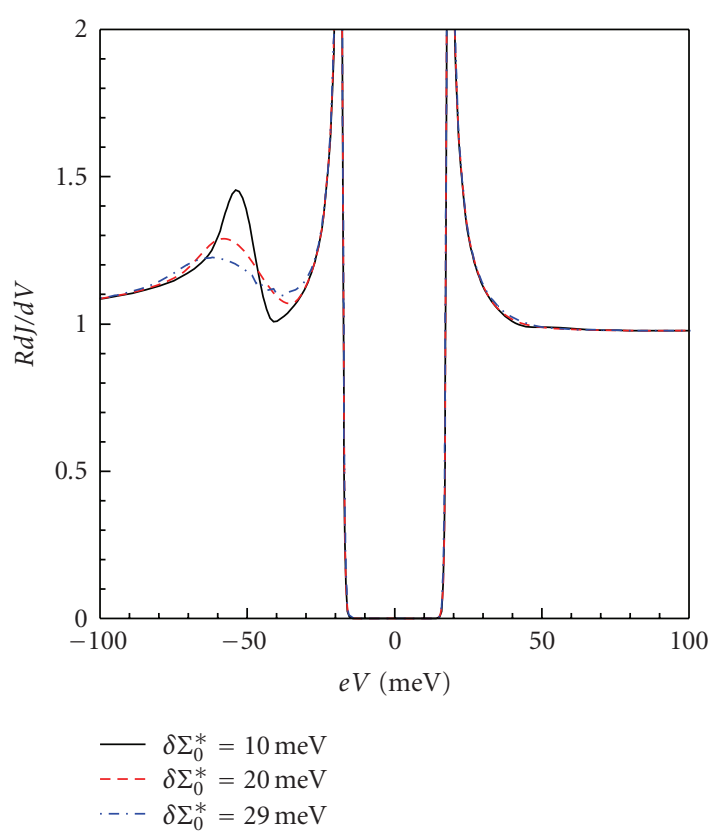

Figure 6: (Color online) Bias voltage, $V$, dependences of the dimensionless differential conductance $R G(V)=R d J / d V$ for the tunnel junction between an inhomogeneous CDW superconductor and a normal metal, expressed in energy units. Here, $J$ is the quasiparticle tunnel current, $R$ is the resistance of the junction in the normal state, and $e>0$ is the elementary charge. The bare parameters of the CDW superconductor are $\Delta_{0}^{*}=20 \mathrm{meV}$, $\Sigma_{0}^{*}=50 \mathrm{meV}$, and $\mu=0.1$; the temperature $T=4.2 \mathrm{~K}$. Various dispersions $\delta \Sigma_{0}^{*}$ centered around the mean value $\Sigma_{0}^{*}=50 \mathrm{meV}$. (Taken from [81].)

An example of the transformation, with $T$, of the DHS-decorated tunnel spectra into the typical pseudogaplike ones is shown in Figure 7 for S-I-N junctions with $\varphi=\pi$ (panel (a)) and $\pi / 2$ (panel (b)). The CDWsuperconductor parameters are $\Delta_{0}=20 \mathrm{meV}, \Sigma_{0}=50 \mathrm{meV}$, $\mu=0.1$, and $\delta \Sigma_{0}=20 \mathrm{meV}$; the temperature $T=$ $4.2 \mathrm{~K}$. For this parameter set, the "actual" superconducting critical temperatures $T_{c}$ of random domains lie within the interval of 114-126 K, and $T_{d}$ is in the range of 197$461 \mathrm{~K}$. From Figure 7(a), the transformation of the DHSincluding pattern of the CVCs calculated for $T \ll T_{c}$ into the pseudogap-like ones in the vicinity of $T_{c}$ or above it becomes clear. The asymmetric curves displayed in (a) are similar to the measured STM $G^{n s}(V)$ dependences for overdoped and underdoped BSCCO compositions [441]. The overall asymmetric slope of the experimental curves, which is independent of gaps and $T$, constitutes the main distinction between them and our theoretical results. It might be connected to the surface charge carrier depletion induced by CDWs and mentioned above. Another interesting feature of our results is a modification and a shift of the $\Delta$ peak. Although $\Delta$ diminishes as $T$ grows, the $\Delta$-peak moves toward higher bias voltages; such a behavior of the $\Delta$-peak is to be undoubtedly associated with its closeness to the $\Sigma$ governed DHS. In experiments, a confusion of identifying this $\Delta$-driven singularity with a pseudogap feature may arise, since the observed transformation of $\Delta$-features into $D$-ones looks very smooth [348].

It is notable that in the case of asymmetric $G^{n s}(V)$, the low- $T$ asymmetry preserves well into the normal state, although the DHS as such totally disappears. The extent of the sample randomness substantially governs CVC patterns. Therefore, pseudogap features might be less or more pronounced for the same materials and doping levels. At the same time, for the reasonable spread of the problem parameters, the superconducting coherent peaks always survive the averaging (below $T_{c}$, of course), in accordance with experiment. Our results also demonstrate that the dependences $\Delta(T)$ taken from the tunnel data may be somewhat distorted in comparison to the true ones due to the unavoidable $\Delta$ versus $\Sigma$ interplay. One should stress that in our model, "hump" positions, which are determined mainly by $\Sigma$ rather than by $\Delta$, anticorrelate with true superconducting gap values $\Delta$ inferred from the coherent peaks of $G(V)$. It is exactly what was found for nonhomogeneous BSCCO samples [614].

Similar CDW-related features should be observed in the CVCs measured for symmetric S-I-S junctions. The $G^{s}(V)$ dependences for this case with the same sets of parameters as in Figure 7 are shown in Figure 8. In analogy with symmetric junctions between BCS superconductors, one would expect an appearance of singularities at $e V= \pm 2 \Delta, \pm(D+\Delta)$, and $\pm 2 \mathrm{D}$. Such, indeed, is the case. However, the magnitudes of the features are quite different (the details of the analysis can be found in $[81,83])$. As readily seen, the transformation of the symmetric DHS pattern into the pseudogap-like picture is similar to that for the nonsymmetric junction. This simplicity is caused by a smallness of the parameter $\mu=0.1$, so that the features at $e V= \pm 2 D$, which are proportional to $\mu^{2}$, are inconspicuous on the chosen scale. At the same time, the singularities at $e V= \pm(D+\Delta)$ are of the square-root type. Note that for arbitrary $\Sigma$ - and $\Delta$-magnitudes, those energies do not coincide with the values $\pm(\Sigma+\Delta)$ (in more frequently used notation, $\left.\pm\left(\Delta_{\mathrm{PG}}+\Delta_{\mathrm{SG}}\right)\right)$, which can be sometimes met in literature [615]. The later relation becomes valid only for $\Sigma \gg \Delta$.

The appearance of the $T$-driven zero-bias peaks is a salient feature of certain CVCs displayed in Figure 8. As is well known [603], this peak is caused by tunneling of thermally excited quasiparticles between empty states with an enhanced DOS located above and below equal superconducting gaps in symmetric S-I-S junctions. Such a feature was found, for example, in $G^{s}(V)$ measured for grainboundary symmetric tunnel junctions in epitaxial films of the $s$-wave oxide CDWS $\mathrm{Ba}_{1-x} \mathrm{~K}_{x} \mathrm{BiO}_{3}$ [616]. One should be careful not to confuse this peak with the dc Josephson peak restricted to $V=0$, which is often seen for symmetric high$T_{c}$ junctions [399]. The distinction consists in the growth of the quasiparticle zero-bias maximum with increasing $T$ up to a certain temperature, followed by its drastic reduction. On the other hand, the Josephson peak decreases monotonously as $T \rightarrow T_{c}$.

The profile and the behavior of the zero-bias peak at nonzero $T$ can be explained in our case by the fact that, in effect, owing to the nonhomogeneity of electrodes, 


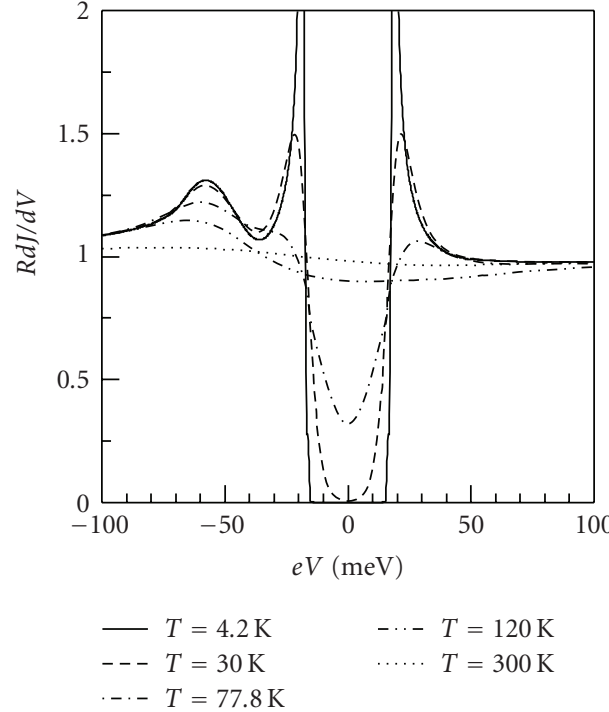

(a)

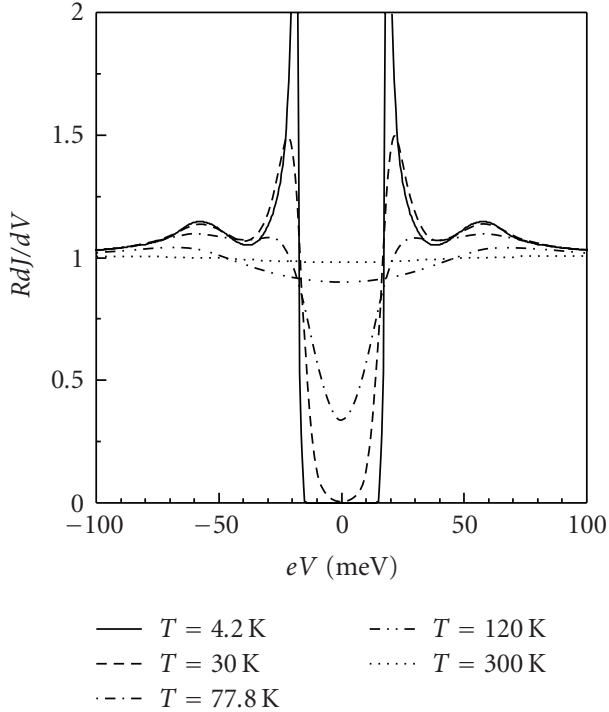

(b)

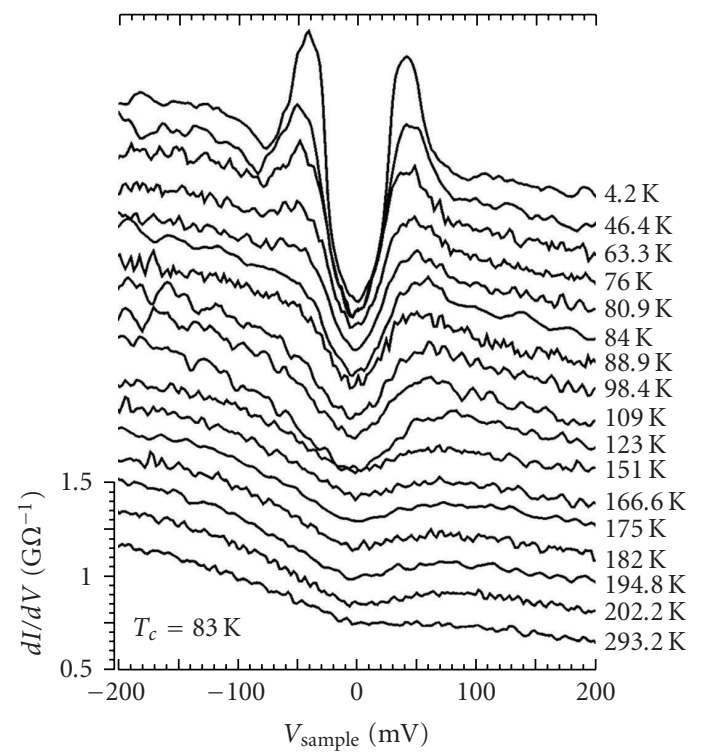

(c)

FIgURE 7: $G(V)$ dependences for the tunnel junction between an inhomogeneous CDWS and a normal metal for various temperatures $T$. The CDW order parameter phase $\varphi=\pi(\mathrm{a})$ and $\pi / 2(\mathrm{~b})$, and the spread of the CDW order parameter-amplitude $\delta \Sigma_{0}^{*}=20 \mathrm{meV}$. All other parameters are indicated in the text. (c) STM spectra for underdoped BSCCO-Ir junctions registered at various temperatures. (Reprinted from [598], taken from [83].)

the junction is a combination of a large number of symmetric and nonsymmetric junctions with varying gap parameters. The former compose a mutual contribution to the current in the vicinity of the $V=0$ point, and the width of this contribution along the $V$-axis is governed by temperature alone. On the other hand, every junction from the latter group gives rise to an elementary current peak in the $\mathrm{CVC}$ at a voltage equal to the relevant gap difference. All such elementary contributions form something like a hump around the zero-bias point, and the width of this hump along the $V$-axis is governed by the sum of actual-dependent on the zero- $T$ values and on the temperature itself-gap spreads in both electrodes. It is clear that the $T$ - behavior of the current contribution of either group is rather complicated, to say nothing of their combination.

From our CVCs calculated for both nonsymmetric (Figures 6 and 7) and symmetric (Figure 8) junctions, it comes about that the "dip" is simply a depression between the hump, which is mainly of the CDW origin, and the superconducting coherent peak. Therefore, as has been noted in [617], the dip has no separate physical meaning. It disappears as $T$ increases, because the coherent peak forming 


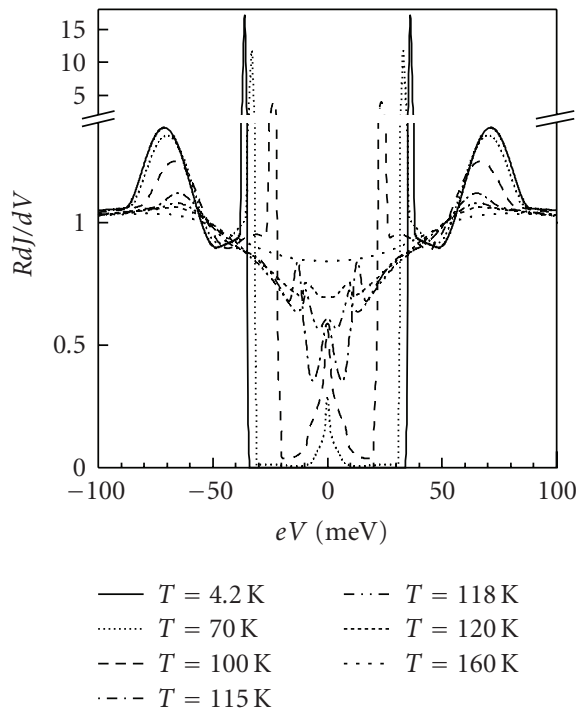

(a)

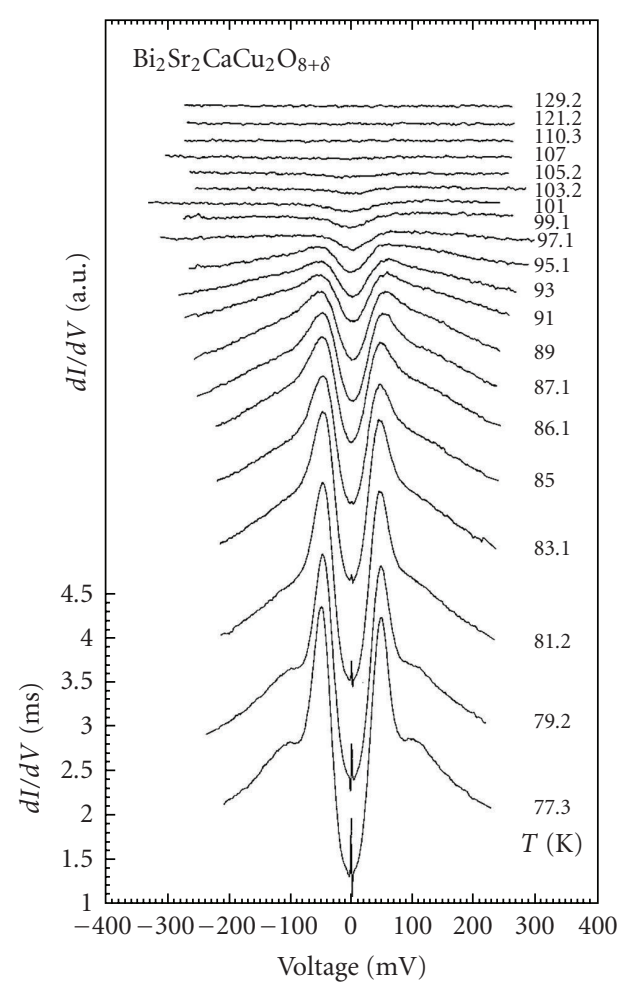

(b)

FIgURE 8: (a) The same as in Figure 7(a), but for a symmetric junction between similar CDW superconductors. (b) Temperature variations of experimental differential current-voltage characteristics (CVCs) for a $\mathrm{Bi}_{2} \mathrm{Sr}_{2} \mathrm{CaCu}_{2} \mathrm{O}_{8+\delta}$ break junction. (Reprinted from [399], taken from [83].)

the other shoulder of the dip fades down, so that the former dip, by expanding to the $V=0$ point, becomes an integral constituent of the shallow pseudogap minimum.

Therefore, it became clear that the CDW manifestations against the nonhomogeneous background can explain both subtle DHS structures in the tunnel spectra for high- $T_{c}$ oxides and large pseudogap features observed both below and above $T_{c}$. The DHS is gradually transformed into the pseudogap-like DOS, lowering as $T$ grows. Hence, both phenomena are closely interrelated, being in essence the manifestations of the same CDW-governed feature smeared by inhomogeneity of CDW superconductors. Therefore, the DHS and pseudogap features should not be treated separately. The dependences of the calculated CVCs on the CDW phase $\varphi$ fairly well describe the variety of asymmetry manifestations in the measured tunnel spectra for BSCCO and related compounds.

4.4. Coexistence of CDWs and d-Wave Superconductivity. We recognize that some of our results, which were obtained assuming that the superconducting order parameter coexisting with CDWs is isotropic, might be applicable to cuprates with certain reservations, since a large body of evidence in favor of $d_{x^{2}-y^{2}}$ symmetry in high- $T_{c}$ oxides $[131,132,305$, $306,618,619$ ] is available, although there are experimentallybased objections $[109,116-129]$. In any case, it seems instructive to extend the partial dielectrization approach to $d$-wave Cooper pairing. For simplicity, we argue in terms of two-dimensional first Brillouin zone and Fermi surface, neglecting $c$-axis quasiparticle dispersion, which should be taken into account, in principle [620]. Since the dielectric, $\Sigma$, and superconducting, $d$-wave $\Delta$, order parameters have different momentum dependences, their joint presence in the electron spectrum is no longer reduced to a combined gap (4), as it was for isotropic superconductivity.

In the $d$-wave case, superconductivity is described by a weak-coupling model with a Hamiltonian given, for example, in [553, 621]. In accordance with photoemission $[371,622-624]$ and STM [359, 368, 370, 506, 507, 512, 625] data (see Figure 1), the mean-field CDW Hamiltonian is restricted to momenta near flat-band regions, antinodal from the viewpoint of the four-lobe $d$-wave gap-function $\Delta(T) \cos 2 \theta$ [306]. In those regions, the nesting conditions (1) between pairs of mutually coupled quasiparticle branches are fulfilled. For instance, static CDW wave vectors $\mathbf{Q}=$ $\left( \pm 2 \pi / 4.2 a_{0}, 0\right)$ and $\left(0, \pm 2 \pi / 4.2 a_{0}\right)$-with an accuracy of $15 \%$-in $\mathrm{Bi}_{2} \mathrm{Sr}_{2} \mathrm{CaCu}_{2} \mathrm{O}_{8+\delta}$ are revealed in STM studies [359]. Thus, we characterize a CDW checkerboard state (symmetric with respect to $\pi / 2$ - rotations) by four sectors in the momentum space centered with the lobes and with an opening $2 \alpha$ each $(\alpha<\pi / 4)$. It should be noted that vectors $\mathbf{Q}$ depend on doping, which was explicitly shown 


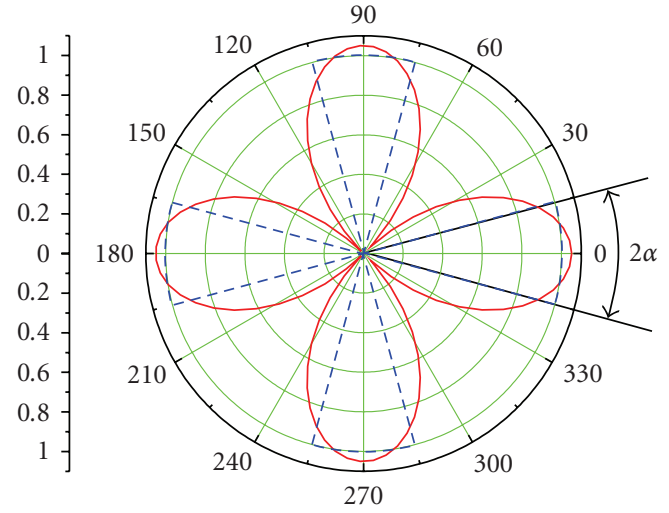

FIgure 9: (Color online) Order-parameter maps for a conventional $d$-wave superconductor $(\Delta$, solid curve) and a partially gapped CDW metal ( $\Sigma$, dashed curve).

for $\mathrm{Bi}_{2} \mathrm{Sr}_{2} \mathrm{CuO}_{6+\delta}$ [343]. The dielectric (CDW-induced) order parameter is $\Sigma(T)$ inside the $2 \alpha$-cones, being angleindependent here, and zero outside (see Figure 9).

The plausibility of this scenario is supported-at least partially-by recent STM studies of intrinsically inhomogeneous BSCCO samples [626]. Specifically, the authors analyzed the composition, temperature, and angular dependences of the gaps on various FS sections and showed that nodal superconducting gaps for overdoped specimens exhibit more or less conventional $d$-wave behavior, whereas in underdoped samples nodal (superconducting) and antinodal gaps (CDW gaps, as is assumed here) superimpose on one another in tunnel spectra. It is important that for underdoped compositions antinodal gaps do not change drastically with $T$, when crossing $T_{c}$. The conclusion made in [626] that the entire FS contributes to bulk superconductivity in overdoped samples corresponds-if proved to be correct-to the actual shrinkage of nested FS sections, that is, to $\mu \rightarrow 0$.

We obtained a new set of Dyson-Gor'kov equations for normal and superconducting Green's functions for the system with electron-hole of whatever nature and $d$-wave Cooper pairings, which were solved in the same straightforward manner as in the $s$-wave case $[160,386]$ (see above). We arrived at the system of two coupled equations for $\Delta(T)$ and $\Sigma(T)$ :

$$
\begin{aligned}
& \int_{0}^{\mu \pi / 4} I_{M}\left(\sqrt{\Sigma^{2}+\Delta^{2} \cos ^{2} 2 \theta}, T, \Sigma_{0}\right) d \theta=0, \\
& \int_{0}^{\mu \pi / 4} I_{M}\left(\sqrt{\Sigma^{2}+\Delta^{2} \cos ^{2} 2 \theta}, T, \Delta_{0}\right) \cos ^{2} 2 \theta d \theta \\
& \quad+\int_{\mu \pi / 4}^{\pi / 4} I_{M}\left(\Delta \cos 2 \theta, T, \Delta_{0}\right) \cos ^{2} 2 \theta d \theta=0,
\end{aligned}
$$

where $\mu=4 \alpha / \pi$ is the dielectrically gapped portion of the FS for the specific model of partial gapping, shown in Figure 9, and $I_{M}\left(\Delta \cos 2 \theta, T, \Delta_{0}\right)$ is the Mühlschlegel integral (6). The analysis of the generic $T-\delta$ phase diagram for cuprates shows that both $\Sigma_{0}$ and $\mu$ reduce with doping, whereas the hole-like FS pockets centered at the $\left(\pi / a_{0}, \pi / a_{0}\right)$ point of the Brillouin zone shrink for every specific high- $T_{c}$ oxide (see, e.g., [343]).
On the other hand, in the absence of CDW gapping, (18) becomes a $d$-wave gap equation:

$$
\int_{0}^{\pi / 4} I_{M}\left(\Delta \cos 2 \theta, T, \Delta_{0}\right) \cos ^{2} 2 \theta d \theta=0
$$

the solution of which $\Delta=d \mathrm{Mu}\left(\Delta_{0}, T\right)$ is known [553, 621]. In particular, the critical temperature is $T_{c 0}=$ $(2 \Omega \gamma / \pi) \exp \left[-1 / V_{\mathrm{BCS}} N(0)\right]$, as in the $s$-wave case. From (19), it follows that in agreement with [553], $\left(\Delta_{0} / T_{c 0}\right)_{d}=$ $(2 / \sqrt{e})(\pi / \gamma)$, revealing a modified " $d$-wave" BCS-ratio different from the $s$-pairing value

$$
\left(\frac{\Delta_{0}}{T_{c 0}}\right)_{s}=\frac{\pi}{\gamma} \approx 0.824\left(\frac{\Delta_{0}}{T_{c 0}}\right)_{d}
$$

Here, $e$ is the base of natural logarithm. It is evident that our model takes into account many-body correlations both explicitly (the emergence of two pairings) and implicitly (via the renormalization of the parameters $\Sigma_{0}$ and $\mu$ ). Weakcoupling values of the ratio $\Delta_{0} / T_{c 0}$ for other anisotropic order parameter symmetries do not differ much from the value of $\left(\Delta_{0} / T_{c 0}\right)_{d}[627,628]$.

Due to the different order parameter symmetry, readily seen from (17) and (18), the situation is mathematically more involved than for isotropic CDW superconductors, where a simple relationship (4) takes place. This was not recognized in a recent work [629], where the opposite wrong statement was made. Prima facie subtle mathematical differences between descriptions of $s$-wave and $d$-wave CDW superconductors lead to conspicuous physical consequences. Indeed, the numerical dependences $\Delta(T)$ and $\Sigma(T)$ found from (17) and (18) and shown in Figure 10 differ qualitatively from their counterparts $\Delta_{s}(T)$ and $\Sigma_{s}(T)$ in a certain range of model parameters. (In this subsection, we do not introduce a natural subscript " $d$ " for brevity.) Figure 10 (a) demonstrates that a reduction of the bare parameter $\Sigma_{0}$, keeping $\Delta_{0}$ and $\mu$ constant, resulting in the transformation of $\Sigma(T)$ with a cusp at $T=T_{c}$ and a concave region at $T<T_{c}$ (the behavior appropriate for CDW $s$-superconductors in the whole allowable parameter range, as is demonstrated in Figure 3) into curves describing a novel peculiar reentrant CDW state. It is remarkable that the reentrance found by us is appropriate to an extremely simple basic model with two competing order parameters. At the same time, the CDW structures in real systems may be much more complicated with nonmonotonic $T$-dependencies even in the absence of superconductivity [352].

Let us formulate conditions necessary to observe this crossover. First, (20) means that $\Delta(T) / \Delta_{0}$ for conventional $d$-superconductors is steeper than $\left(\Delta(T) / \Delta_{0}\right)_{s}$. In our case, it means that $\Delta(T) / \Delta_{0}$, when the CDW disappears, is steeper than $\Sigma(T) / \Sigma_{0}$ in the absence of superconductivity, which is described by (6). Hence, for the CDW phase to exist (the upper critical temperature $T_{\mathrm{CDW}}^{u}>0$ ), it should be $T_{\mathrm{CDW}}^{u}=(\gamma / \pi) \Sigma_{0}>T_{c 0}=(\sqrt{e} \gamma / 2 \pi) \Delta_{0}$. As a consequence, the first constraint on the model parameters should be fulfilled: $\Sigma_{0}>(\sqrt{e} / 2) \Delta_{0} \approx 0.824 \Delta_{0}$. The constraint stems from the competition between emerging $\Delta$ and $\Sigma$ on the $d$ FS section only. The actual coexistence between superconductivity and 


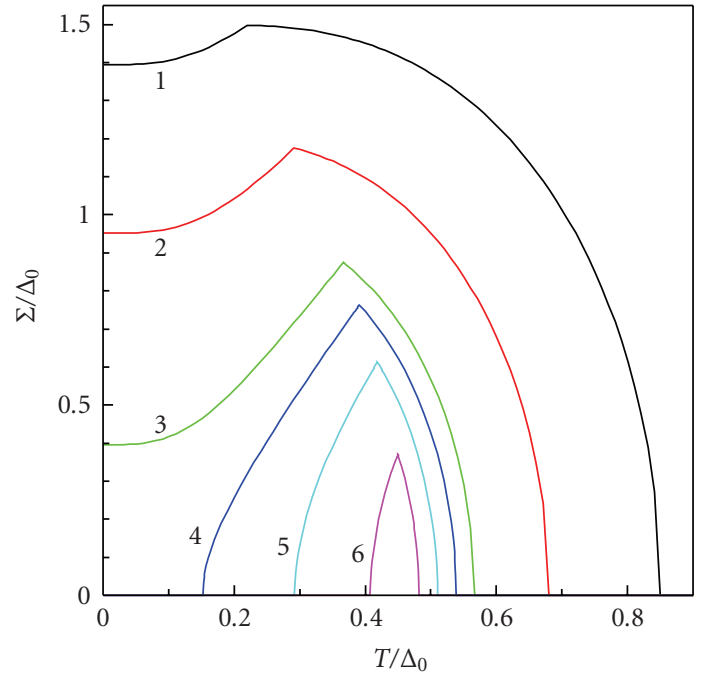

(a)

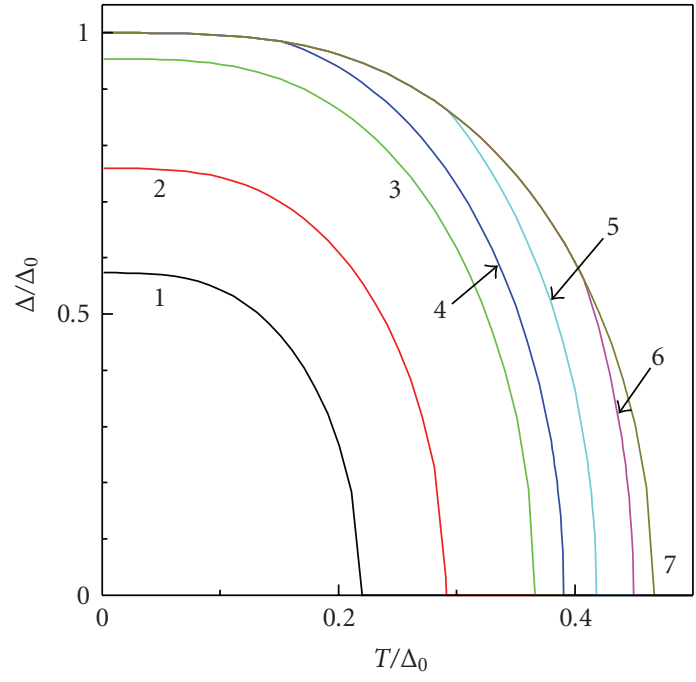

(b)

FIgure 10: (Color online) Temperature, $T$, dependences of the normalized (a) CDW $\Sigma$ and (b) superconducting $\Delta$ gap functions. $\Delta_{0}$ equal to $\Delta(T=0)$ when CDWs are absent is 1 . The values of $\Sigma_{0} / \Delta_{0}$ equal to $\Sigma(T=0) / \Delta_{0}$ in the absence of superconductivity are $1.5(1), 1.2(2), 1$ (3), $0.95(4), 0.9(5), 0.85(6)$, and $0.8(7) ; \mu=0.3$.

CDWs was not involved in these reasonings, so the inequality does not include the control parameter $\mu$. Therefore, $T_{\mathrm{CDW}}^{u}$ thus defined coincides with $T_{\mathrm{CDW} 0}$.

Second, below the lower critical temperature of the CDW reentrance region, $T_{\mathrm{CDW}}^{l}$, if any, (18) defines $\Delta(T)=$ $d \mathrm{Mü}\left(\Delta_{0}, T\right)$, and we should use (17) with $T=T_{\mathrm{CDW}}^{l}$ and $\Delta\left(T_{\mathrm{CDW}}^{l}\right)=d \mathrm{Mü}\left(\Delta_{0}, T_{\mathrm{CDW}}^{l}\right)$ to determine $T_{\mathrm{CDW}}^{l}\left(\Delta_{0}, \Sigma_{0}, \mu\right)$ numerically. The crossover value of $\Sigma_{0}^{\mathrm{cr}}$, when $T_{\mathrm{CDW}}^{l}=$ 0 , corresponds to the separatrix on the order parameter$T$ plane, dividing possible $\Sigma(T)$-curves (see Figure 10(a)) into two types: reentrant and nonreentrant. However, (17) brings about $\Sigma_{0}^{\mathrm{cr}}=\Delta_{0} \exp \left[(4 / \mu \pi) \int_{0}^{\mu \pi / 4} \ln (\cos 2 \theta) d \theta\right]$. To observe the reentrant behavior, the second constraint should be $\Sigma<\Sigma_{0}^{\text {cr }}$. For the curves in Figure 10, $\mu=0.3$ was chosen, so that we obtain the reentrance range $0.824 \Delta_{0}<$ $\Sigma_{0}<0.963 \Delta_{0}$, which agrees with numerical solutions of the full self-consistent equation set. We emphasize that CDWs survives the competition with $d$-wave superconductivity even at $\Sigma_{0} / \Delta_{0}<1$, which is not the case for stronger isotropic Cooper pairing (see the discussions above).

In Figure 10(b), the concomitant $\Delta(T)$ dependences are depicted. One sees how $d$-wave superconductivity, suppressed at large $\Sigma_{0}$, recovers in the reentrance parameter region. Therefore, two regimes of CDW manifestation can be observed in superconductors. In both cases, the CDW is seen as a pseudogap above $T_{c}[81,83]$ in photoemission and tunnel experiments. However, the corresponding DHS at low $T$ may either be observed or not, depending on whether the reentrance occurs. This might be an additional test for an anisotropic (not necessarily $d_{x^{2}-y^{2}}$-wave) Cooper pairing to dominate in cuprates.

To control the change-over between different regimes in cuprates, one can use either hydrostatic pressure or doping. In both cases, $\mu$ is the main varying parameter. In Figure 11, the curves $\Sigma(T)$ and $\Delta(T)$ are shown for $\Sigma_{0} / \Delta_{0}=0.9$ and various $\mu$. It is readily seen how drastic is the low- $T$ depression of $\Sigma$ by superconductivity, when the dielectrically gapped FS sectors are small enough. Doping $\mathrm{Bi}_{2} \mathrm{Sr}_{2} \mathrm{CaCu}_{2} \mathrm{O}_{8+\delta}$ [403] and $(\mathrm{Bi}, \mathrm{Pb})_{2}(\mathrm{Sr}, \mathrm{La})_{2} \mathrm{CuO}_{6+\delta}$ [356] with oxygen was shown to sharply shrink the parameter $\mu$. Note that the $\Delta(T)$ dependences are distorted by CDWs, and they do not coincide with the scaled "parent" curve- $d \mathrm{Mu}(T)$, in this case-in contrast to what should be observed for CDW $s$-superconductors (Figure 3). Therefore, various observed forms of $\Delta(T)$ per se cannot unambiguously testify to the superconducting pairing symmetry. Moreover, cuprate superconductivity might be, for example, a mixture of $s$ - and $d$-wave contributions [130,630].

It is evident that different strengths of CDW-imposed suppression of the superconducting energy gap in the electron spectrum $\Delta$ and the critical temperature $T_{c}$ must change the ratio $\Delta(0) / T_{c}$ - the benchmark of weak-coupling superconductivity (see (20)). If one recalls that, as was shown above, this ratio in CDW $s$-superconductors remains the same as in conventional $s$-ones, the situation becomes very intriguing. In Figure 12(a), the dependences of $2 \Delta(0) / T_{c}$ and $T_{c} / \Delta_{0}$ ratios on $\Sigma_{0} / \Delta_{0}$ are displayed. One sees that $2 \Delta(0) / T_{c}$ sharply increases with $\Sigma_{0} / \Delta_{0}$ for $\Sigma_{0} / \Delta_{0} \leq 1$ and swiftly saturates for larger $\Sigma_{0} / \Delta_{0}$, whereas $T_{c} / \Delta_{0}$ decreases almost evenly. The saturation value proves to be 5.2 for $\mu=0.3$. We stress that such large enhancement of $2 \Delta(0) / T_{c}$ agrees well with experimental data $[441,478,631,632]$ for cuprates and cannot be achieved taking into account strongcoupling electron-boson interaction effects for reasonable relationships between $T_{c}$ and effective boson frequencies $\omega_{E}$ $[633,634]$ (one can hardly accept, e.g., the value $T_{c} / \omega_{E} \approx$ 0.3 [634] as practically meaningful). Furthermore, the destruction of the alternating-sign superconducting order 


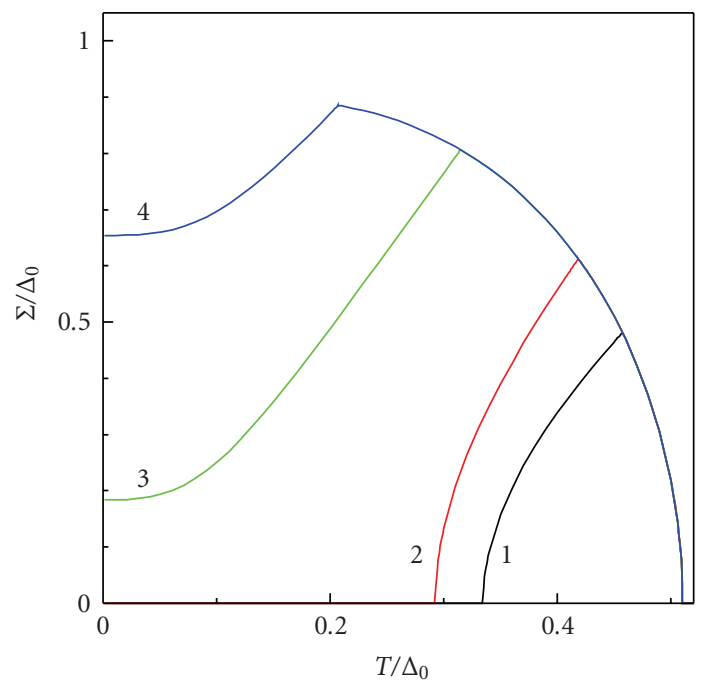

(a)

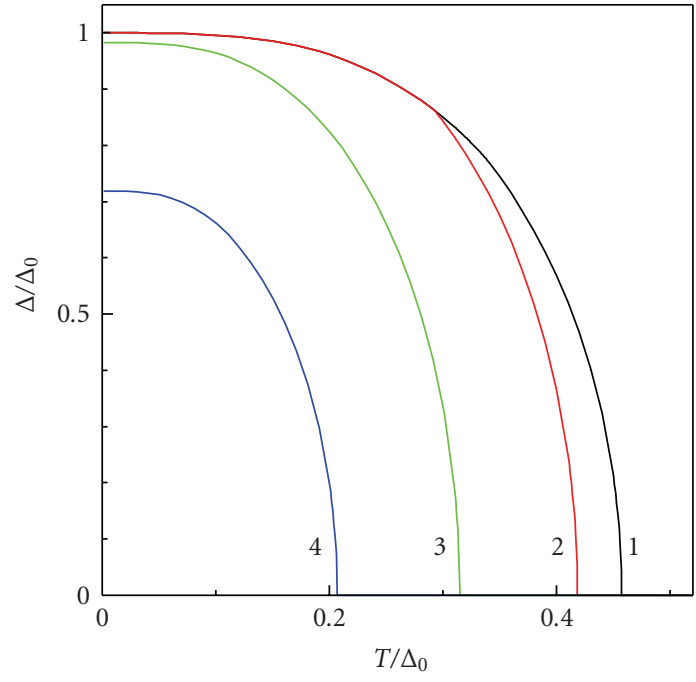

(b)

FIgURE 11: (Color online) The same as in Figure 10 but for $\Sigma_{0} / \Delta_{0}=0.9$ and $\mu=0.1$ (1), 0.3 (2), 0.5 (3), 0.6 (4).

parameter by impurity scattering approximated by collective boson modes also could not explain [635] high values of $2 \Delta(0) / T_{c}$, for example, inherent to underdoped BSCCO $[347,597]$. Therefore, our weak-coupling model is sufficient to explain —on its own - the large magnitude of $2 \Delta(0) / T_{c}$ in cuprates, possible strong-coupling effects resulting in at most minor corrections.

Another possible alternative reason of high $2 \Delta(0) / T_{c}$ ratios might be a singular energy dependence of the normalstate electron DOS near the FS, for instance, near the Van Hove anomalies in low-dimensional electron subsystems [518]. It turned out, however, that, at least in the weakcoupling (BCS) approximation for $s$-wave Cooper pairing, the ratio $2 \Delta(0) / T_{c}$ is not noticeably altered [636, 637]. Moreover, calculations in the framework of the strongcoupling Eliashberg theory [10] showed that the van Hove singularity influence on $T_{c}$ is even smaller than in the BCS limit [638]. Furthermore, weak-coupling calculations for orthorhombically distorted hole-doped cuprate superconductors (without CDWs) demonstrated that $2 \Delta(0) / T_{c}$ can be estimated as an intermediate between $s$-wave and $d$ wave limits [639], being smaller than needed to explain the experiment. It means that our approach remains so far the only one capable of explaining high $2 \Delta(0) / T_{c} \approx 5 \div 8$ (and even larger values [632]) for cuprates. We emphasize that it is very important to reconcile theoretical values for $2 \Delta(0) / T_{c}$ as well as $\Delta C / \gamma_{S} T_{c}$ with experimental ones. Otherwise, the microscopic theory becomes "too" phenomenological with $\Delta / T_{c}$ as an additional free parameter of the system [640].

It is instructive from the methodological point of view to mention a previous unsuccessful attempt to explain the increase of $2 \Delta(0) / T_{c}$ by a pseudogap influence [641]. The authors of this reference assumed the identical $d$ wave symmetry for both the superconducting, $\Delta(T)$, and temperature-independent pseudogap, $E_{\mathrm{PG}}$, order parameters. Additionally, dielectric gapping was supposed to be effectively complete rather than partial, the latter being intrinsic to our model and follows from the experiments for cuprates. These circumstances excluded self-consistency from the approach and led to superfluous restrictions imposed on $E_{\mathrm{PG}}$, namely, $E_{\mathrm{PG}} \lesssim 0.53 \Delta_{0}(T=0)$, where $\Delta_{0}(T=0)$ is the parent superconducting order parameter amplitude. At the same time, it is well known that for existing CDW superconductors the strength of CDW instability is at least not weaker than that of its Cooper-pairing counterpart [160]. We should emphasize once more that the main peculiarity of our model, dictated by the observations, which led to the adequate description of thermodynamic properties for $d$-wave superconductors with CDWs, is the distinction between relevant order parameter symmetries.

The $\mu$-dependences of $2 \Delta(0) / T_{c}$ and $T_{c} / \Delta_{0}$ are shown in Figure $12(\mathrm{~b})$. They illustrate that $2 \Delta(0) / T_{c}$ can reach rather large values, if the dielectric gapping sector is wide enough. This growth is however limited by a drastic drop of $T_{c}$ leading to a quick disappearance of superconductivity. We think that it is exactly the case of underdoped cuprates, when a decrease of $T_{c}$ is accompanied by a conspicuous widening of the superconducting gap. For instance, such a scenario was clearly observed in break-junction experiments for $\mathrm{Bi}_{2} \mathrm{Sr}_{2} \mathrm{CaCu}_{2} \mathrm{O}_{8+\delta}$ samples with a large doping range [642].

As was pointed out in [478], various photoemission and tunneling measurements for different cuprate families show a typical average value $2 \Delta(0) / T_{c} \approx 5.5$. From Figure $12($ b), we see that this ratio corresponds to $\mu \approx 0.35$ at $\Sigma_{0} / \Delta_{0}=1$. The other curve readily gives $T_{c} / \Delta_{0} \approx 0.35$. Since $\Delta_{0} / T_{c 0} \approx$ 2.14 for a $d$-wave superconductor (see above), we obtain $T_{c} / T_{c 0} \approx 0.75$, being quite a reasonable estimation of $T_{c^{-}}$ reduction by CDWs. 


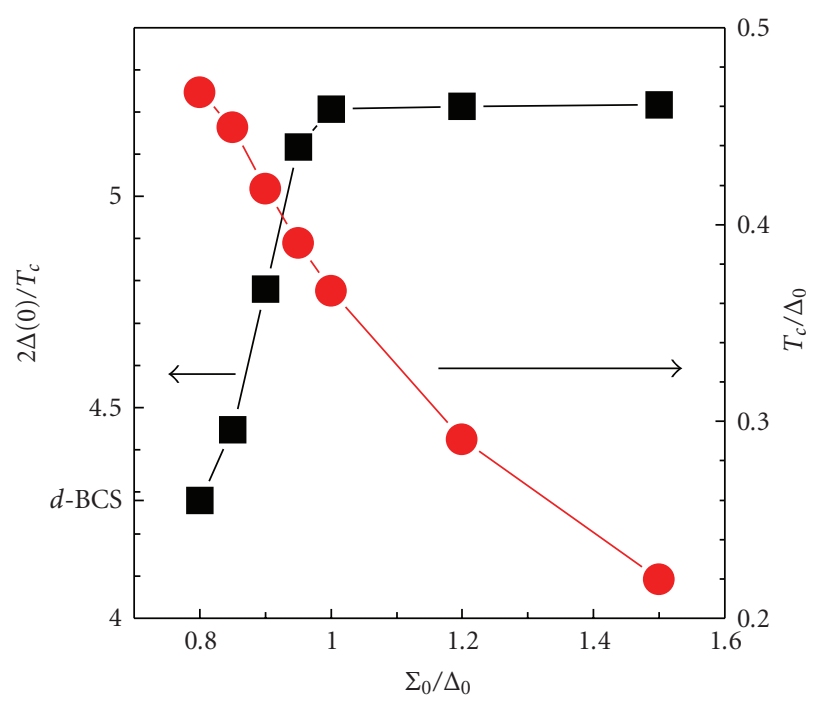

(a)

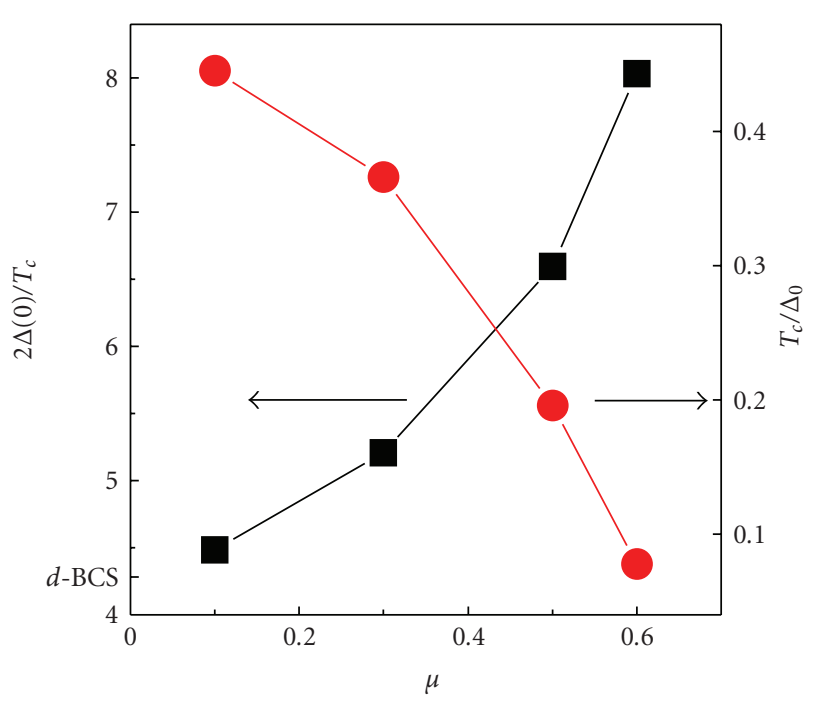

(b)

Figure 12: (Color online) Dependences of $2 \Delta(0) / T_{c}$ (squares) and $T_{c} / \Delta_{0}$ (circles) on $\Sigma_{0} / \Delta_{0}$ (panel (a), $\mu=0.3$ ) and $\mu$ (panel (b), $\Delta_{0} / \Sigma_{0}=1$ ). $T_{c}$ is the superconducting critical temperature, $d-\mathrm{BCS} \approx 4.28$ is a value for a conventional superconductor with $d$-wave symmetry of the order parameter.

4.5. SDWs and Superconductivity. There are plenty of materials, where SDWs compete with superconductivity, although a simultaneous existence of the order parameters cannot be always proved [160, 643, 644]. For completeness, we give here certain short comments on the latest developments in this direction.

During the last years, an interest arose to the phase with the hidden order parameter in $\mathrm{URu}_{2} \mathrm{Si}_{2}$, emerging at about $17.5 \mathrm{~K}$ and being some kind of SDWs, coexisting with superconductivity at $T<1.5 \mathrm{~K}$ [153, 645-647]. It should be noted that the partial gapping idea applied to SDW materials [397, 422-424, 566, 648-650] was invoked to explain ordering in this compound at the times of the discovery [651].

More attention was paid to $\mathrm{Cr}$ and its alloys, where CDWs and SDWs are linked and coexist $[652,653]$. It might be interesting to observe mutual influence of CDWs and SDWs on superconductivity [654-657].

The problem of an interplay between SDWs and superconductivity received strong impetus recently, especially because of the fundamental discovery of magnetic elementbased high- $T_{c}$ pnictide superconductors [49, 654-657]. Theoretical efforts were also continued (see, e.g., [658662]). It is worthwhile noting that, for certain doping ranges, superconducting cuprates also demonstrate [663] the coexistence of Cooper pairing with SDWs rather than CDWs, the latter being appropriate for the majority of high- $T_{c}$ oxide compositions (see above and [160]).

Although the coexistence of superconductivity with SDWs or more exotic orbital antiferromagnetic and spin current ordering $[522,548,664]$ is left beyond the scope of this review, the relevant physics is not less fascinating than that of their CDW-involving analogues.

\section{Conclusions}

The presented material testifies that CDWs play the important role in high- $T_{c}$ oxides and govern some of the properties that usually have been considered as solely determined by superconductivity per se. Sometimes CDWs manifest themselves explicitly (observed checkerboard or unidirectional structures, DHSs, psedudogaps) but, in the majority of phenomena, they "only" — but often drastically_change the magnitude of certain effects in the superconducting state (the heat capacity anomaly, the paramagnetic limit, the $T$-dependence of $H_{c 2}$, the $\Delta(0) / T_{c}$ ratio). Cuprates are not unique as materials with coexisting CDWs and superconductivity, but the scale of the interplay is very large here due to the strength of the Cooper pairing in those compounds.

\section{Acknowledgments}

The authors are grateful to Antonio Bianconi, Sergei Borisenko, Ilya Eremin, Peter Fulde, Stefan Kirchner, Alexander Kordyuk, Dirk Manske, and Kurt Scharnberg for useful discussions. A. M. Gabovich and A. I. Voitenko are also grateful to Kasa im. Józefa Mianowskiego, Polski Koncern Naftowy ORLEN, and Fundacja Zygmunta Zaleskiego as well as to project no. 23 of the 2009-2011 Scientific Cooperation Agreement between Poland and Ukraine for the financial support of their visits to Warsaw. A. M. Gabovich highly appreciates the 2008 and 2009 Visitors Programs of the Max Planck Institute for the Physics of Complex Systems (Dresden, Germany). T. Ekino was partly supported by a Grantin-Aid for Scientific Research (nos. 19540370, 19105006, 19014016) of Japan Society of Promotion of Science. M. S. Li 
was supported by the Ministry of Science and Informatics in Poland (grant no. 202-204-234).

\section{References}

[1] J. G. Bednorz and K. A. Müller, "Possible high $T_{c}$ superconductivity in the Ba-La-Cu-O system," Zeitschrift für Physik B, vol. 64, no. 2, pp. 189-193, 1986.

[2] P. W. Anderson, The Theory of Superconductivity in the High$T_{c}$ Cuprates, Princeton Univeristy Press, Princeton, NJ, USA, 1997.

[3] V. P. Mineev and K. V. Samokhin, Intoduction to Unconventional Superconductivity, Gordon and Breach Science, Amsterdam, The Netherlands, 1999.

[4] M. L. Kulić, "Interplay of electron-phonon interaction and strong correlations: the possible way to high-temperature superconductivity," Physics Reports, vol. 338, no. 1-2, pp. 1264, 2000.

[5] S. L. Drechsler, H. Rosner, J. Málek, and H. Eschrig, "Electronic and magnetic properties of cuprate chains and related materials. From bandstructure to aspects of many-body physics in real materials," in High- $T_{c}$ Superconductors and Related Materials. Material Science, Fundamental Properties, and Some Future Electronic Applications, S. L. Drechsler and T. Mishonov, Eds., p. 81, Kluwer Academic Publishers, Dordrecht, The Netherlands, 2001.

[6] N. M. Plakida, Spectroscopy of High-T, Superconductors: A Theoretical View, Taylor and Francis, New York, NY, USA, 2003.

[7] Y. Yanase, T. Jujo, T. Nomura, H. Ikeda, T. Hotta, and K. Yamada, "Theory of superconductivity in strongly correlated electron systems," Physics Reports, vol. 387, no. 1-4, pp. 1149, 2003.

[8] A. S. Alexandrov, Theory of Superconductivity: From Weak to Strong Coupling, IOP, Philadelphia, Pa, USA, 2003.

[9] L. Pitaevskii, "Theoretical foundation: phenomenology and microscopic theory," in The Physics of Superconductors. Vol. 1: Conventional and High- $T_{c}$ Superconductors, K. H. Bennemann and J. B. Ketterson, Eds., p. 23, Springer, Berlin, Germany, 2003.

[10] J. P. Carbotte and F. Marsiglio, "Electron-phonon superconductivity," in The Physics of Superconductors. Vol. 1: Conventional and High- $T_{c}$ Superconductors, K. H. Bennemann and J. B. Ketterson, Eds., p. 233, Springer, Berlin, Germany, 2003.

[11] A. V. Chubukov, D. Pines, and J. Schmalian, "A spin fluctuation model for $d$-wave superconductivity," in The Physics of Superconductors. Vol. 1: Conventional and High- $T_{c}$ Superconductors, K. H. Bennemann and J. B. Ketterson, Eds., p. 495, Springer, Berlin, Germany, 2003.

[12] S. A. Kivelson, I. P. Bindloss, E. Fradkin, et al., "How to detect fluctuating stripes in the high-temperature superconductors," Reviews of Modern Physics, vol. 75, no. 4, pp. 12011241, 2003.

[13] D. Manske, Theory of Unconventional Superconductors. Cooper-Pairing Mediated by Spin Excitations, Springer, New York, NY, USA, 2004.

[14] E. W. Carlson, V. J. Emery, S. A. Kivelson, and D. Orgad, "Concepts in high temperature superconductivity," in The Physics of Superconductors. Vol. 2: Conventional and High- $T_{c}$ Superconductors, K. H. Bennemann and J. B. Ketterson, Eds., p. 275, Springer, Berlin, Germany, 2004.

[15] D. Manske, I. Eremin, and K. H. Bennemann, "Electronic theory for superconductivity in high- $T_{c}$ cuprates and
$\mathrm{Sr}_{2} \mathrm{RuO}_{4}$," in The Physics of Superconductors. Vol. 2: Conventional and High- $T_{c}$ Superconductors, K. H. Bennemann and J. B. Ketterson, Eds., p. 731, Springer, Berlin, Germany, 2004.

[16] A. J. Leggett, "Superfluid ${ }^{3} \mathrm{He}$ and the cuprate superconductors," in The Physics of Superconductors. Vol. 2: Conventional and High- $T_{c}$ Superconductors, K. H. Bennemann and J. B. Ketterson, Eds., p. 1087, Springer, Berlin, Germany, 2004.

[17] M. L. Kulić, "Electron-phonon interaction and strong correlations in high-temperature superconductors: One can not avoid the unavoidable," in Lectures on the Physics of Highly Correlated Electron Systems VIII: Eighth Training Course, A. Avella and F. Mancini, Eds., p. 75, American Institute of Physics, Melville, NY, USA, 2004.

[18] M. Eschrig, "The effect of collective spin-1 excitations on electronic spectra in high- $T_{c}$ superconductors," Advances in Physics, vol. 55, no. 1-2, pp. 47-183, 2006.

[19] A. V. Balatsky, I. Vekhter, and J.-X. Zhu, "Impurity-induced states in conventional and unconventional superconductors," Reviews of Modern Physics, vol. 78, no. 2, pp. 373-433, 2006.

[20] A.-M. S. Tremblay, B. Kyung, and D. Sénéchal, "Pseudogap and high-temperature superconductivity from weak to strong coupling. Towards quantitative theory," Fizika Nizkikh Temperatur, vol. 32, no. 4-5, pp. 561-595, 2006.

[21] B. Edegger, V. N. Muthukumar, and C. Gros, "GutzwillerRVB theory of high-temperature superconductivity: results from renormalized mean-field theory and variational Monte Carlo calculations," Advances in Physics, vol. 56, no. 6, pp. 927-1033, 2007.

[22] V. Barzykin and D. Pines, "Universal behaviour and the twocomponent character of magnetically underdoped cuprate superconductors," Advances in Physics, vol. 58, no. 1, pp. 165, 2009.

[23] J. Hauck and K. Mika, "Classification of superconducting oxide structures," Superconductor Science and Technology, vol. 8, no. 5, pp. 374-381, 1995.

[24] Z. Fisk and J. L. Sarrao, "The new generation hightemperature superconductors," Annual Review of Materials Science, vol. 27, no. 1, pp. 35-67, 1997.

[25] J. Hauck and K. Mika, "Structure families of superconducting oxides and interstitial alloys," Superconductor Science and Technology, vol. 11, no. 7, pp. 614-630, 1998.

[26] R. J. Cava, "Oxide superconductors," Journal of the American Ceramic Society, vol. 83, no. 1, pp. 5-28, 2000.

[27] H. R. Ott, "High- $T_{c}$ superconductivity," in The Physics of Superconductors. Vol. 1: Conventional and High- $T_{c}$ Superconductors, K. H. Bennemann and J. B. Ketterson, Eds., p. 385, Springer, Berlin, Germany, 2003.

[28] J. R. Gavaler, M. A. Janocko, and C. K. Jones, "Preparation and properties of high- $T_{c} \mathrm{Nb}-\mathrm{Ge}$ films," Journal of Applied Physics, vol. 45, no. 7, pp. 3009-3013, 1974.

[29] L. R. Testardi, "Structural instability and superconductivity in A-15 compounds," Reviews of Modern Physics, vol. 47, no. 3, pp. 637-648, 1975.

[30] K. Holczer and R. L. Whetten, "Superconducting and normal state properties of the $\mathrm{A}_{3} \mathrm{C}_{60}$ compounds," Carbon, vol. 30, no. 8, pp. 1261-1276, 1992.

[31] O. Gunnarsson, "Superconductivity in fullerides," Reviews of Modern Physics, vol. 69, no. 2, pp. 575-606, 1997.

[32] R. J. Cava, B. Batlogg, J. J. Krajewski, et al., "Superconductivity near $30 \mathrm{~K}$ without copper: the $\mathrm{Ba}_{0.6} \mathrm{~K}_{0.4} \mathrm{BiO}_{3}$ perovskite," Nature, vol. 332, no. 6167, pp. 814-816, 1988.

[33] L. F. Mattheiss, E. M. Gyorgy, and D. W. Johnson Jr., "Superconductivity above $20 \mathrm{~K}$ in the Ba-K-Bi-O system," Physical Review B, vol. 37, no. 7, pp. 3745-3746, 1988. 
[34] G.-M. Zhao, "Muon spin relaxation and magnetic measurements on $\mathrm{Ba}_{0.63} \mathrm{~K}_{0.37} \mathrm{BiO}_{3}$ : evidence for polaronic strongcoupling phonon-mediated pairing," Physical Review B, vol. 76, no. 2, Article ID 020501, 4 pages, 2007.

[35] S. Yamanaka, K. Hotehama, and H. Kawaji, "Superconductivity at $25.5 \mathrm{~K}$ in electron-doped layered hafnium nitride," Nature, vol. 392, no. 6676, pp. 580-582, 1998.

[36] S. Yamanaka, "High- $T_{c}$ superconductivity in electron-doped layer structured nitrides," Annual Review of Materials Science, vol. 30, pp. 53-82, 2000.

[37] J. Nagamatsu, N. Nakagawa, T. Muranaka, Y. Zenitani, and J. Akimitsu, "Superconductivity at $39 \mathrm{~K}$ in magnesium diboride," Nature, vol. 410, no. 6824, pp. 63-64, 2001.

[38] J. Akimitsu and T. Muranaka, "Superconductivity in $\mathrm{MgB}_{2}$," Physica C, vol. 388-389, pp. 98-102, 2003.

[39] V. A. Drozd, A. M. Gabovich, P. Gierłowski, M. Pȩkała, and H. Szymczak, "Transport properties of bulk and thin-film $\mathrm{MgB}_{2}$ superconductors: effects of preparation conditions," Physica C, vol. 402, no. 4, pp. 325-334, 2004.

[40] X. X. Xi, "Two-band superconductor magnesium diboride," Reports on Progress in Physics, vol. 71, no. 11, Article ID 116501, 26 pages, 2008.

[41] S. Reich, G. Leitus, R. Popovitz-Biro, A. Goldbourt, and S. Vega, "A possible $2 \mathrm{D} \mathrm{H}_{x} \mathrm{WO}_{3}$ superconductor with a $T_{c}$ of 120 K," Journal of Superconductivity and Novel Magnetism, vol. 22, no. 4, pp. 343-346, 2009.

[42] A. M. Gabovich, V. A. Drozd, M. Pȩkała, T. Ekino, and R. Ribeiro, "Competition between superconductivity and charge carrier localization in plumbates," in Superconductivity Research Advances, J. E. Nolan, Ed., p. 149, Nova Science, New York, NY, USA, 2007.

[43] H. Takahashi, K. Igawa, K. Arii, Y. Kamihara, M. Hirano, and $\mathrm{H}$. Hosono, "Superconductivity at $43 \mathrm{~K}$ in an ironbased layered compound $\mathrm{LaO}_{1-x} \mathrm{~F}_{x} \mathrm{FeAs}$," Nature, vol. 453, no. 7193, pp. 376-378, 2008.

[44] H. Hosono, "Layered iron pnictide superconductors: discovery and current status," Journal of the Physical Society of Japan, vol. 77, pp. 1-8, 2008.

[45] M. Rotter, M. Tegel, and D. Johrendt, "Superconductivity at $38 \mathrm{~K}$ in the iron arsenide $\left(\mathrm{Ba}_{1-x} \mathrm{~K}_{x}\right) \mathrm{Fe}_{2} \mathrm{As}_{2}$," Physical Review Letters, vol. 101, no. 10, Article ID 107006, 4 pages, 2008.

[46] A. L. Ivanovskii, "New high-temperature superconductors based on rare earth and transition metal oxyarsenides and related phases: synthesis, properties and simulation," Uspekhi Fizicheskikh Nauk, vol. 178, p. 1273, 2008.

[47] T. C. Ozawa and S. M. Kauzlarich, "Chemistry of layered dmetal pnictide oxides and their potential as candidates for new superconductors," Science and Technology of Advanced Materials, vol. 9, no. 3, Article ID 033003, 11 pages, 2008.

[48] H. Hosono, "Two classes of superconductors discovered in our material research: iron-based high temperature superconductor and electride superconductor," Physica C, vol. 469, no. 9-12, pp. 314-325, 2009.

[49] C. Day, "Iron-based superconductors," Physics Today, vol. 62, no. 8, pp. 36-40, 2009.

[50] V. L. Ginzburg, "About ferromagnetic superconductors," Zhurnal Eksperimental'noi i Teoreticheskoi Fiziki, vol. 31, p. 202, 1956.

[51] M. B. Maple and $\varnothing$. Fischer, Eds., Superconductivity in Ternary Compounds II, Superconductivity and Magnetism, vol. 34 of Topics in Current Physics, Springer, Berlin, Germany, 1982.

[52] L. N. Bulaevskii, A. I. Buzdin, M. L. Kulić, and S. V. Panjukov, "Coexistence of superconductivity and magnetism theoretical predictions and experimental results," Advances in Physics, vol. 34, no. 2, pp. 175-261, 1985.

[53] L. N. Bulaevskii, "Magnetic superconductors," in Superconductivity, Superdiamagnetism, Superfluidity, V. L. Ginzburg, Ed., p. 69, Mir, Moscow, Russia, 1987.

[54] Yu. A. Izyumov, M. I. Katsnelson, and Yu. N. Skryabin, Itinerant Electron Magnetism, Fiziko- Matematicheskaya Literatura, Moscow, Russia, 1994.

[55] A. I. Buzdin, "Proximity effects in superconductorferromagnet heterostructures," Reviews of Modern Physics, vol. 77, no. 3, pp. 935-976, 2005.

[56] A. M. Gabovich and D. P. Moiseev, "Metalloxide superconductor $\mathrm{BaPb}_{1-x} \mathrm{Bi}_{x} \mathrm{O}_{3}$ : unusual properties and new applications," Uspekhi Fizicheskikh Nauk, vol. 150, p. 599, 1986.

[57] R. Collongues, La Non-Stoechiometrie, Masson, Paris, France, 1971.

[58] A. M. Stoneham and L. W. Smith, "Defect phenomena in superconducting oxides and analogous ceramic oxides," Journal of Physics: Condensed Matter, vol. 3, no. 3, pp. 225278, 1991.

[59] O. Matsumoto, A. Utsuki, A. Tsukada, H. Yamamoto, T. Manabe, and M. Naito, "Synthesis and properties of superconducting $T-R_{2} \mathrm{CuO}_{4}(R=\mathrm{Pr}, \mathrm{Nd}, \mathrm{Sm}, \mathrm{Eu}, \mathrm{Gd})$," Physical Review B, vol. 79, no. 10, Article ID 100508, 4 pages, 2009.

[60] Z. Ren, Q. Tao, S. Jiang, et al., "Superconductivity induced by phosphorus doping and its coexistence with ferromagnetism in $\mathrm{EuFe}_{2}\left(\mathrm{As}_{0.7} \mathrm{P}_{0.3}\right)_{2}$," Physical Review Letters, vol. 102, no. 13, Article ID 137002, 4 pages, 2009.

[61] R. J. Cava, "Contemporary superconducting materials," Chemical Communications, no. 43, pp. 5373-5377, 2005.

[62] W. E. Pickett, "The next breakthrough in phonon-mediated superconductivity," Physica C, vol. 468, no. 2, pp. 126-135, 2008.

[63] J. C. Phillips, "Hard-wired dopant networks and the prediction of high transition temperatures in ceramic superconductors," Advances in Condensed Matter Physics, vol. 2010, Article ID 250891, 13 pages, 2010.

[64] J. C. Phillips, "Zigzag filamentary theory of longitudinal optical phonons in high-temperature superconductors," Philosophical Magazine B, vol. 81, no. 1, pp. 35-53, 2001.

[65] J. C. Phillips and J. Jung, "Nanodomain structure and function of high-temperature superconductors," Philosophical Magazine B, vol. 81, no. 8, pp. 745-756, 2001.

[66] J. C. Phillips, "Percolative model of nanoscale phase separation in high-temperature superconductors," Philosophical Magazine B, vol. 82, no. 7, pp. 783-790, 2002.

[67] J. C. Phillips, "Self-organized networks and lattice effects in high-temperature superconductors," Physical Review B, vol. 75, no. 21, Article ID 214503, 23 pages, 2007.

[68] G. Litak, A. M. Martin, B. Györffy, J. F. Annett, and K. I. Wysokiñski, "Van Hove singularity and $d$-wave pairing in disordered superconductors," Physica C, vol. 309, no. 3-4, pp. 257-262, 1998.

[69] G. Litak and B. Györffy, "Random negative-U hubbard model," Physical Review B, vol. 62, no. 10, pp. 6629-6637, 2000.

[70] G. Litak, B. Györffy, and K. I. Wysokiñski, "Charge and order parameter fluctuations in disordered superconductors," Physica C, vol. 308, no. 1-2, pp. 132-146, 1998.

[71] G. Litak, "Charge and phase fluctuations in attractive Hubbard model," Physica C, vol. 387, no. 1-2, pp. 86-88, 2003. 
[72] G. Litak, "Charge order versus superconductivity in inhomogeneous systems," Journal of Superconductivity and Novel Magnetism, vol. 22, no. 3, pp. 265-267, 2009.

[73] A. M. Gabovich and A. I. Voitenko, "Temperature-dependent inelastic electron scattering and superconducting state properties," Physics Letters A, vol. 190, no. 2, pp. 191-195, 1994.

[74] A. M. Gabovich and A. I. Voitenko, "Influence of inelastic quasiparticle scattering on thermodynamic and transport properties of high- $T_{c}$ oxides," Physica $C$, vol. 258, no. 3-4, pp. 236-252, 1996.

[75] A. M. Gabovich, "Power-law low-temperature asymptotics for spatially nonhomogeneous s-wave superconductors," Fizika Nizkikh Temperatur, vol. 25, no. 7, pp. 677-684, 1999.

[76] A. M. Gabovich and A. I. Voitenko, "Influence of orderparameter nonhomogeneities on low-temperature properties of superconductors," Physical Review B, vol. 60, no. 10, pp. 7465-7472, 1999.

[77] A. M. Gabovich, A. I. Voitenko, M. S. Li, and H. Szymczak, "Heat capacity of mesoscopically disordered superconductors: implications for $\mathrm{MgB}_{2}$," Fizika Nizkikh Temperatur, vol. 28, no. 11, pp. 1126-1137, 2002.

[78] A. M. Gabovich, M. S. Li, M. Pękała, H. Szymczak, and A. I. Voitenko, "Heat capacity of mesoscopically disordered superconductors with emphasis on $\mathrm{MgB}_{2}$," Journal of Physics: Condensed Matter, vol. 14, no. 41, pp. 9621-9629, 2002.

[79] A. M. Gabovich, M. S. Li, M. Pȩkała, H. Szymczak, and A. I. Voitenko, "Heat capacity of mesoscopically inhomogeneous superconductors: theory and applications to $\mathrm{MgB}_{2}$," Physica C, vol. 405, no. 3-4, pp. 187-211, 2004.

[80] T. Ekino, A. M. Gabovich, M. S. Li, et al., "Spatially heterogeneous character of superconductivity in $\mathrm{MgB}_{2}$ as revealed by local probe and bulk measurements," Physica $C$, vol. 426-431, no. 1, pp. 230-233, 2005.

[81] A. M. Gabovich and A. I. Voitenko, "Charge-density-wave origin of the dip-hump structure in tunnel spectra of the BSCCO superconductor," Physical Review B, vol. 75, no. 6, Article ID 064516, 13 pages, 2007.

[82] T. Ekino, A. M. Gabovich, M. S. Li, M. Pȩkała, H. Szymczak, and A. I. Voitenko, "Analysis of the pseudogaprelated structure in tunneling spectra of superconducting $\mathrm{Bi}_{2} \mathrm{Sr}_{2} \mathrm{CaCu}_{2} \mathrm{O}_{8+\delta}$ revealed by the break-junction technique," Physical Review B, vol. 76, no. 18, Article ID 180503, 4 pages, 2007.

[83] T. Ekino, A. M. Gabovich, M. S. Li, M. Pękała, H. Szymczak, and A. I. Voitenko, "Temperature-dependent pseudogaplike features in tunnel spectra of high- $T_{c}$ cuprates as a manifestation of charge-density waves," Journal of Physics: Condensed Matter, vol. 20, no. 42, Article ID 425218, 15 pages, 2008.

[84] J. C. Phillips, "Is there a lowest upper bound for superconductive transition temperatures?" Journal of Physics: Conference Series, vol. 108, no. 1, Article ID 012033, 6 pages, 2008.

[85] B. T. Matthias, "Anticorrelations in superconductivity," Physica, vol. 55, pp. 69-72, 1971.

[86] F. E. Wang and M. A. Mitchell, "A new correlation: superconducting critical temperature vs number of naturally occuring isotopes among superconducting elements," Solid State Communications, vol. 15, no. 5, pp. 867-869, 1974.

[87] D. M. Gualtieri, "The correlation between the superconducting critical temperature and the number of stable isotopes among superconducting elements," Solid State Communications, vol. 16, no. 7, pp. 917-918, 1975.
[88] I. O. Kulik, "Superconductivity and macroscopic stability criteria for electron-phonon systems," Zhurnal Eksperimental'noj i Teoreticheskoj Fiziki, vol. 66, p. 2224, 1974.

[89] I. O. Kulik, "Spin fluctuations and superconductivity," Fizika Nizkikh Temperatur, vol. 2, p. 486, 1976.

[90] A. M. Gabovich, "On the criterion of superconductivity of metals," Ukrainskii Fizychnyi Zhurnal, vol. 22, p. 2072, 1977.

[91] A. M. Gabovich and D. P. Moiseev, "Superconductivity of metals with the allowance for ion core repulsion," Fizika Nizkikh Temperatur, vol. 4, p. 1115, 1978.

[92] A. M. Gabovich and D. P. Moiseev, "Isotope effect in jellium and Brout models," Fizika Tverdogo Tela, vol. 23, p. 1511, 1981.

[93] I. M. Chapnik, "On the possible relation between the absence of superconductivity in Au and Pd-Ag alloys and the negative sign of the Hall coefficient," Journal of Physics F, vol. 13, no. 5, pp. 975-978, 1983.

[94] I. M. Chapnik, "Regularities in the occurrence of superconductivity," Journal of Physics F, vol. 14, no. 8, pp. 1919-1921, 1984.

[95] J. E. Hirsch, "Correlations between normal-state properties and superconductivity," Physical Review B, vol. 55, no. 14, pp. 9007-9024, 1997.

[96] C. Buzea and K. Robbie, "Assembling the puzzle of superconducting elements: a review," Superconductor Science and Technology, vol. 18, no. 1, pp. R1-R8, 2005.

[97] F. Huber, H. P. Roeser, and M. von Schoenermark, "A correlation between $T_{c}$ of Fe-based HT superconductors and the crystal super lattice constants of the doping element positions," Journal of the Physical Society of Japan, vol. 77, pp. 142-144, 2008.

[98] B. J. Taylor and M. B. Maple, "Formula for the critical temperature of superconductors based on the electronic density of states and the effective mass," Physical Review Letters, vol. 102, no. 13, Article ID 137003, 4 pages, 2009.

[99] A. M. Gabovich, "About superconductivity of polar semiconductors," Fizika Tverdogo Tela, vol. 22, no. 11, pp. 3231-3235, 1980.

[100] R. Baquero, "What determines the magnitude of $T_{c}$ in HTSC?" in Frontiers in Contemporary Physics-EAV08, L. M. M. Zetina, G. T. Vega, M. G. Roclia, L. F. R. Oclioa, and R. L. Fernández, Eds., p. 218, American Institute of Physics, Melville, NY, USA, 2008.

[101] X. Blase, E. Bustarret, C. Chapelier, T. Klein, and C. Marcenat, "Superconducting group-IV semiconductors," Nature Materials, vol. 8, no. 5, pp. 375-382, 2009.

[102] B. E. C. Koltenbah and R. Joynt, "Material-specific gap function in the $t-J$ model of high-temperature superconductors," Reports on Progress in Physics, vol. 60, no. 1, pp. 23-56, 1997.

[103] M. Ogata and H. Fukuyama, "The $t-J$ model for the oxide high- $T_{c}$ superconductors," Reports on Progress in Physics, vol. 71, no. 3, Article ID 036501, 45 pages, 2008.

[104] W. Fan, "Predictions of highest transition-temperature for electron-phonon superconductors," Physica C, vol. 469, no. 4, pp. 177-181, 2009.

[105] G. M. Eliashberg, "Interaction of electrons with lattice vibrations in a superconductor," Zhurnal Eksperimental'noi i Teoreticheskoi Fiziki, vol. 38, p. 966, 1960.

[106] G. M. Eliashberg, “Temperature Green's functions of electrons in a superconductor," Zhurnal Eksperimental'noi $i$ Teoreticheskoi Fiziki, vol. 39, p. 1437, 1960.

[107] B. Brandow, "Characteristic features of the exotic superconductors," Physics Reports, vol. 296, no. 1, pp. 1-63, 1998. 
[108] B. Brandow, "Explanation of the exotic superconductors by a valence-fluctuation pairing mechanism," Philosophical Magazine B, vol. 80, no. 6, pp. 1229-1297, 2000.

[109] G.-M. Zhao, "Experimental constraints on the physics of cuprates," Philosophical Magazine B, vol. 81, no. 10, pp. 1335$1388,2001$.

[110] G.-M. Zhao, "Pairing interactions and pairing mechanism in high-temperature copper oxide superconductors," Physical Review B, vol. 71, no. 10, Article ID 104517, 9 pages, 2005.

[111] A. S. Alexandrov, "High temperature superconductivity due to a long-range electron-phonon interaction, application to isotope effects, thermomagnetic transport and nanoscale heterogeneity in cuprates," Journal of Superconductivity, vol. 18, no. 5-6, pp. 603-612, 2005.

[112] W. E. Pickett, "Design for a room-temperature superconductor," Journal of Superconductivity and Novel Magnetism, vol. 19, no. 3-5, pp. 291-297, 2006.

[113] A. M. Kadin, "Coherent lattice vibrations in superconductors," Physica C, vol. 468, no. 4, pp. 255-259, 2008.

[114] J. Tahir-Kheli and W. A. Goddard III, "The Chiral Plaquette Polaron Paradigm (CPPP) for high temperature cuprate superconductors," Chemical Physics Letters, vol. 472, no. 46, pp. 153-165, 2009.

[115] V. Z. Kresin and S. A. Wolf, "Colloquium: electron-lattice interaction and its impact on high $T_{c}$ superconductivity," Reviews of Modern Physics, vol. 81, no. 2, pp. 481-501, 2009.

[116] C. Panagopoulos and T. Xiang, "Relationship between the superconducting energy gap and the critical temperature in high- $T_{c}$ superconductors," Physical Review Letters, vol. 81, no. 11, pp. 2336-2339, 1998.

[117] R. A. Klemm and K. Scharnberg, "Theory of bicrystal $c$-axis twist Josephson junctions," International Journal of Modern Physics B, vol. 15, no. 24-25, pp. 3164-3189, 2001.

[118] G.-M. Zhao, "Identification of the bulk pairing symmetry in high-temperature superconductors: evidence for an extended $s$ wave with eight line nodes," Physical Review B, vol. 64, no. 2, Article ID 024503, 10 pages, 2001.

[119] B. H. Brandow, "Arguments and evidence for a nodecontaining anisotropic $s$-wave gap form in the cuprate superconductors," Physical Review B, vol. 65, no. 5, Article ID 054503, 5 pages, 2002.

[120] B. H. Brandow, "Strongly anisotropic s-wave gaps in exotic superconductors," Philosophical Magazine, vol. 83, no. 21, pp. 2487-2519, 2003.

[121] G.-M. Zhao, "The magnetic resonance in high-temperature superconductors: evidence for an extended $s$-wave pairing symmetry," Philosophical Magazine B, vol. 84, no. 36, pp. 3869-3882, 2004.

[122] R. A. Klemm, "The phase-sensitive $c$-axis twist experiments on $\mathrm{Bi}_{2} \mathrm{Sr}_{2} \mathrm{CaCu}_{2} \mathrm{O}_{8+\delta}$ and their implications," Philosophical Magazine, vol. 85, no. 8, pp. 801-853, 2005.

[123] T. H. Geballe, "The never-ending search for hightemperature superconductivity," Journal of Superconductivity and Novel Magnetism, vol. 19, no. 3-5, pp. 261-276, 2006.

[124] R. Baquero, "Superconductivity today: a brief panorama," in Advanced Summer School in Physics 2005, O. Rosas-Ortiz, M. Carbajal, and O. Miranda, Eds., p. 301, American Institute of Physics, Melville, NY, USA, 2006.

[125] R. Khasanov, S. Strässle, D. Di Castro, et al., "Multiple gap symmetries for the order parameter of cuprate superconductors from penetration depth measurements," Physical Review Letters, vol. 99, no. 23, Article ID 237601, 4 pages, 2007.

[126] T. Das, R. S. Markiewicz, and A. Bansil, "Nodeless $d$-wave superconducting pairing due to residual antiferromagnetism in underdoped $\operatorname{Pr}_{2-x} \mathrm{Ce}_{x} \mathrm{CuO}_{4-\delta}$," Physical Review Letters, vol. 98, no. 19, Article ID 197004, 4 pages, 2007.

[127] G.-M. Zhao, "Precise determination of the superconducting gap along the diagonal direction of $\mathrm{Bi}_{2} \mathrm{Sr}_{2} \mathrm{CaCu}_{2} \mathrm{O}_{8+y}$ : evidence for an extended $s$-wave gap symmetry," Physical Review $B$, vol. 75, no. 14, Article ID 140510, 4 pages, 2007.

[128] R. Khasanov, A. Shengelaya, A. Maisuradze, et al., "Nodeless superconductivity in the infinite-layer electron-doped cuprate superconductor $\mathrm{Sr}_{0.9} \mathrm{La}_{0.1} \mathrm{CuO}_{2}$," Physical Review B, vol. 77, no. 18, Article ID 184512, 6 pages, 2008.

[129] R. Prozorov, "Superfluid density in a superconductor with an extended $d$-wave gap," Superconductor Science and Technology, vol. 21, no. 8, Article ID 082003, 3 pages, 2008.

[130] M. Bakr, A. P. Schnyder, L. Klam, et al., "Electronic and phononic Raman scattering in detwinned $\mathrm{YBa}_{2} \mathrm{Cu}_{3} \mathrm{O}_{6.95}$ and $\mathrm{Y}_{0.85} \mathrm{Ca}_{0.15} \mathrm{Ba}_{2} \mathrm{Cu}_{3} \mathrm{O}_{6.95}$ : s-wave admixture to the $d_{x^{2}-y^{2}}$-wave order parameter," Physical Review B, vol. 80, no. 6, Article ID 064505, 11 pages, 2009.

[131] C. C. Tsuei and J. R. Kirtley, "Pairing symmetry in cuprate superconductors," Reviews of Modern Physics, vol. 72, no. 4, pp. 969-1016, 2000.

[132] H. Hilgenkamp and J. Mannhart, "Grain boundaries in high$T_{c}$ superconductors," Reviews of Modern Physics, vol. 74, no. 2, pp. 485-549, 2002.

[133] C. C. Tsuei and J. R. Kirtley, "Pairing symmetry in cuprate superconductors: phase-sensitive tests," in The Physics of Superconductors. Vol. 1: Conventional and High- $T_{c}$ Superconductors, K. H. Bennemann and J. B. Ketterson, Eds., p. 647, Springer, Berlin, Germany, 2003.

[134] R. Micnas, J. Ranninger, and S. Robaszkiewicz, "Superconductivity in narrow-band systems with local nonretarded attractive interactions," Reviews of Modern Physics, vol. 62, no. 1, pp. 113-171, 1990.

[135] A. S. Alexandrov, "Polaronic and bipolaronic high- $T_{c}$ superconductivity," in Models and Phenomenology for Conventional and High-Temperature Superconductivity, G. Iadonisi, J. R. Schrieffer, and M. L. Chiofalo, Eds., p. 309, IOS Press, Amsterdam, The Netherlands, 1998.

[136] T. Skośkiewicz, A. W. Szafrañski, W. Bujnowski, and B. Baranowski, "Isotope effect in the superconducting palladiumhydrogen-deuterium system," Journal of Physics C, vol. 7, no. 15, pp. 2670-2676, 1974.

[137] B. Stritzker, "Ion implantation and superconductivity," Journal of Nuclear Materials, vol. 72, no. 1-2, pp. 256-262, 1978.

[138] T. Matsubara and A. Kotani, Eds., Superconductivity in Magnetic and Exotic Materials, Springer, Berlin, Germany, 1984.

[139] A. Taraphder, R. Pandit, H. R. Krishnamurthy, and T. V. Ramakrishnan, "The exotic barium bismuthates," International Journal of Modern Physics B, vol. 10, no. 8, pp. 863-955, 1996.

[140] A. M. Gabovich, "Possibility of cold fusion in palladium deutendes: screening effects and connection to superconducting properties," Philosophical Magazine B, vol. 76, no. 1, pp. 107118, 1997.

[141] N. D. Mathur, F. M. Grosche, S. R. Julian, et al., "Magnetically mediated superconductivity in heavy fermion compounds," Nature, vol. 394, no. 6688, pp. 39-43, 1998.

[142] E. Bauer, G. Hilscher, H. Michor, et al., "Heavy fermion superconductivity and magnetic order in noncentrosymmet-

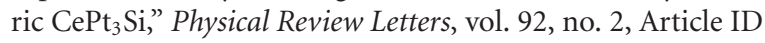
027003, 4 pages, 2004. 
[143] S. G. Ovchinnikov, "Exotic superconductivity and magnetism in ruthenates," Uspekhi Fizicheskikh Nauk, vol. 173, no. 1, pp. 27-51, 2003.

[144] A. P. Mackenzie and Y. Maeno, "The superconductivity of $\mathrm{Sr}_{2} \mathrm{RuO}_{4}$ and the physics of spin-triplet pairing," Reviews of Modern Physics, vol. 75, no. 2, pp. 657-712, 2003.

[145] P. S. Riseborough, G. M. Schmiedeschoff, and J. L. Smith, "Heavy fermion superconductivity," in The Physics of Superconductors. Vol. 2: Conventional and High- $T_{c}$ Superconductors, K. H. Bennemann and J. B. Ketterson, Eds., p. 889, Springer, Berlin, Germany, 2004.

[146] P. Thalmeier, G. Zwicknagl, O. Stockert, G. Sparn, and F. Steglich, "Superconductivity in heavy Fermion compounds," in Frontiers in Superconducting Materials, A. V. Narlikar, Ed., p. 109, Springer, Berlin, Germany, 2005.

[147] D. A. Bonn, "Are high-temperature superconductors exotic?" Nature Physics, vol. 2, no. 3, pp. 159-166, 2006.

[148] S. Fujimoto, "Electron correlation and pairing states in superconductors without inversion symmetry," Journal of the Physical Society of Japan, vol. 76, no. 5, Article ID 051008, 14 pages, 2007.

[149] Y. Kitaoka, H. Mukuda, M. Yashima, and A. Harada, "Unconventional pairing states in heavy-fermion superconductors studied by the NQR/NMR experiments," Journal of the Physical Society of Japan, vol. 76, no. 5, Article ID 051001, 15 pages, 2007.

[150] T. Sakakibara, A. Yamada, J. Custers, et al., "Nodal structures of heavy fermion superconductors probed by the specificheat measurements in magnetic fields," Journal of the Physical Society of Japan, vol. 76, no. 5, Article ID 051004, 11 pages, 2007.

[151] J. L. Sarrao and J. D. Thompson, "Superconductivity in cerium- and plutonium-based '115' materials," Journal of the Physical Society of Japan, vol. 76, no. 5, Article ID 051013, 9 pages, 2007.

[152] Y. Haga, H. Sakai, and S. Kambe, "Recent advances in the 5frelevant electronic states and unconventional superconductivity of actinide compounds," Journal of the Physical Society of Japan, vol. 76, no. 5, Article ID 051012, 21 pages, 2007.

[153] Y. Kasahara, T. Iwasawa, H. Shishido, et al., "Gap structure and exotic superconducting state of $\mathrm{URu}_{2} \mathrm{Si}_{2}$," Journal of Physics: Conference Series, vol. 150, Article ID 052098, 4 pages, 2009.

[154] R. D. Parks, Ed., Superconductivity, vol. 1, Marcel Dekker, New York, NY, USA, 1969.

[155] R. D. Parks, Ed., Superconductivity, vol. 2, Marcel Dekker, New York, NY, USA, 1969.

[156] K. H. Bennemann and J. B. Ketterson, Eds., The Physics of Superconductors. Vol. 1: Conventional and High- $T_{c}$ Superconductors, Springer, Berlin, Germany, 2003.

[157] K. H. Bennemann and J. B. Ketterson, Eds., The Physics of Superconductors. Vol. 2: Conventional and High- $T_{c}$ Superconductors, Springer, Berlin, Germany, 2004.

[158] A. M. Gabovich and A. I. Voitenko, "Superconductors with charge- and spin-density waves: theory and experiment," Fizika Nizkikh Temperatur, vol. 26, no. 5, pp. 419-452, 2000.

[159] A. M. Gabovich, A. I. Voitenko, J. F. Annett, and M. Ausloos, "Charge- and spin-density-wave superconductors," Superconductor Science and Technology, vol. 14, no. 4, pp. R1R27, 2001.

[160] A. M. Gabovich, A. I. Voitenko, and M. Ausloos, "Chargeand spin-density waves in existing superconductors: competition between Cooper pairing and Peierls or excitonic instabilities," Physics Reports, vol. 367, no. 6, pp. 583-709, 2002 .
[161] I. I. Mazin and J. Schmalian, "Pairing symmetry and pairing state in ferropnictides: theoretical overview," Physica $C$, vol. 469, no. 9-12, pp. 614-627, 2009.

[162] A. W. Sleight, J. L. Gillson, and P. E. Bierstedt, "Hightemperature superconductivity in the $\mathrm{BaPb}_{1-x} \mathrm{Bi}_{x} \mathrm{O}_{3}$ systems," Solid State Communications, vol. 17, no. 1, pp. 27-28, 1975.

[163] S. Tajima, S. Uchida, A. Masaki, et al., "Electronic states of $\mathrm{BaPb}_{1-x} \mathrm{Bi}_{x} \mathrm{O}_{3}$ in the semiconducting phase investigated by optical measurements," Physical Review B, vol. 35, no. 2, pp. 696-703, 1987.

[164] S. Tajima, H. Ishii, I. Rittaporn, et al., "Vacuum-ultraviolet spectra and band structure of $\mathrm{BaPb}_{1-x} \mathrm{Bi}_{x} \mathrm{O}_{3}$," Physical Review B, vol. 38, no. 2, pp. 1143-1147, 1988.

[165] D. T. Marx, P. G. Radaelli, J. D. Jorgensen, et al., "Metastable behavior of the superconducting phase in the $\mathrm{BaBi}_{1-x} \mathrm{~Pb}_{x} \mathrm{O}_{3}$ system," Physical Review B, vol. 46, no. 2, pp. 1144-1156, 1992.

[166] M. Yasukawa and N. Murayama, "A phase transition with an abrupt electrical resistivity change around $800 \mathrm{~K}$ in $\mathrm{BaBi}_{0.5} \mathrm{~Pb}_{0.5} \mathrm{O}_{3}$," Physica C, vol. 297, no. 3-4, pp. 326-332, 1998.

[167] A. Yamamoto, T. Furumochi, and S. Tajima, "Metal-insulator transition and superconductivity in Sr- and La-substituted $\mathrm{BaPb}_{1-x} \mathrm{Bi}_{x} \mathrm{O}_{3}$," Physica C, vol. 328, no. 1, pp. 118-124, 1999.

[168] V. J. Emery and S. A. Kivelson, "Superconductivity in bad metals," Physical Review Letters, vol. 74, no. 16, pp. 32533256, 1995.

[169] N. F. Mott and E. A. Davis, Electron Processes in NonCrystalline Materials, Clarendon Press, Oxford, UK, 1979.

[170] J. F. Schooley, W. R. Hosler, E. Ambler, J. H. Becker, M. L. Cohen, and C. S. Koonce, "Dependence of the superconducting transition temperature on carrier concentration in semiconducting $\mathrm{SrTiO}_{3}$," Physical Review Letters, vol. 14, no. 9, pp. 305-307, 1965.

[171] K. Shibuya, T. Ohnishi, T. Sato, and M. Lippmaa, "Metalinsulator transition in $\mathrm{SrTiO}_{3}$ induced by field effect," Journal of Applied Physics, vol. 102, no. 8, Article ID 083713, 2007.

[172] C. H. Ahn, A. Bhattacharya, M. Di Ventra, et al., "Electrostatic modification of novel materials," Reviews of Modern Physics, vol. 78, no. 4, pp. 1185-1212, 2006.

[173] A. Ohtomo and H. Y. Hwang, "A high-mobility electron gas at the $\mathrm{LaAIO}_{3} / \mathrm{SrTiO}_{3}$ heterointerface," Nature, vol. 427, no. 6973, pp. 423-426, 2004.

[174] N. Reyren, S. Thiel, A. D. Caviglia, et al., "Superconducting interfaces between insulating oxides," Science, vol. 317, no. 5842, pp. 1196-1199, 2007.

[175] S. Gariglio, N. Reyren, A. D. Caviglia, and J.-M. Triscone, "Superconductivity at the $\mathrm{LaAIO}_{3} / \mathrm{SrTiO}_{3}$ interface," Journal of Physics: Condensed Matter, vol. 21, no. 16, Article ID 164213, 2009.

[176] L. J. de Jongh, "A comparative study of (BI)polaronic (super)conductivity in high- and low- $T_{c}$ superconducting oxides," Physica C, vol. 152, no. 2, pp. 171-216, 1988.

[177] T. Egami and S. J. L. Billinge, "Lattice effects in high temperature superconductors," Progress in Materials Science, vol. 38, no. 1, pp. 359-424, 1994.

[178] K. S. Aleksandrov and B. V. Beznosikov, "Hierarchy of perovskite-like crystals:a review," Fizika Tverdogo Tela, vol. 39, p. 785, 1997.

[179] K. S. Aleksandrov and J. Bartolomé, "Structural distortions in families of perovskite-like crystals," Phase Transitions, vol. 74, no. 3, pp. 255-335, 2001. 
[180] M. Karppinen and H. Yamauchi, "Control of the charge inhomogeneity and high- $T_{c}$ superconducting properties in homologous series of multi-layered copper oxides," Materials Science and Engineering R, vol. 26, no. 3, pp. 51-96, 1999.

[181] T. Yamauchi, M. Isobe, and Y. Ueda, "Charge order and superconductivity in vanadium oxides," Solid State Sciences, vol. 7, no. 7, pp. 874-881, 2005.

[182] A. S. Barker Jr. and M. Tinkham, "Far-infrared ferroelectric vibration mode in $\mathrm{SrTiO}_{3}$," Physical Review, vol. 125, no. 5, pp. 1527-1530, 1962.

[183] M. E. Lines and A. M. Glass, Principles and Application of Ferroelectrics and Related Materials, Clarendon Press, Oxford, UK, 1977.

[184] C. Z. Bi, J. Y. Ma, J. Yan, et al., "Electron-phonon coupling in $\mathrm{Nb}$-doped $\mathrm{SrTiO}_{3}$ single crystal," Journal of Physics: Condensed Matter, vol. 18, no. 8, pp. 2553-2561, 2006.

[185] D. M. Eagles, "Superconductivity at very low carrier concentrations and indications of a charged bose gas in $\mathrm{SrTi}_{0.97} \mathrm{Zr}_{0.03} \mathrm{O}_{3}$," Solid State Communications, vol. 60, no. 6, pp. 521-524, 1986.

[186] M. Jourdan, N. Blümer, and H. Adrian, "Superconductivity of $\mathrm{SrTiO}_{3-\delta}$," European Physical Journal B, vol. 33, no. 1, pp. 25-30, 2003.

[187] M. Jourdan and H. Adrian, "Possibility of unconventional superconductivity of $\mathrm{SrTiO}_{3-\delta}$," Physica C, vol. 388-389, pp. 509-510, 2003.

[188] V. L. Vinetskii, "Bipolar states of current carriers in ionic crystals," Zhurnal Eksperimental'noi i Teoreticheskoi Fiziki, vol. 40, p. 1459, 1961.

[189] J. Bardeen, L. N. Cooper, and J. R. Schrieffer, "Theory of superconductivity," Physical Review, vol. 108, no. 5, pp. 1175 1204, 1957.

[190] M. R. Schafroth, S. T. Butler, and J. M. Blatt, "Quasichemical equilibrium approach to superconductivity," Helvetica Physica Acta, vol. 30, p. 93, 1957.

[191] D. M. Eagles, "Possible pairing without superconductivity at low carrier concentrations in bulk and thin-film superconducting semiconductors," Physical Review, vol. 186, no. 2, pp. 456-463, 1969.

[192] A. S. Alexandrov and J. Ranninger, "Theory of bipolarons and bipolaronic bands," Physical Review B, vol. 23, no. 4, pp. 1796-1801, 1981.

[193] A. S. Alexandrov and J. Ranninger, "Bipolaronic superconductivity," Physical Review B, vol. 24, no. 3, pp. 1164-1169, 1981.

[194] A. S. Alexandrov, J. Ranninger, and S. Robaszkiewicz, "Bipolaronic superconductivity: thermodynamics, magnetic properties, and possibility of existence in real substances," Physical Review B, vol. 33, no. 7, pp. 4526-4542, 1986.

[195] D. Emin and M. S. Hillery, "Formation of a large singlet bipolaron: application to high-temperature bipolaronic superconductivity," Physical Review B, vol. 39, no. 10, pp. 6575-6593, 1989.

[196] R. Friedberg, T. D. Lee, and H. C. Ren, "A correction to Schafroth's superconductivity solution of an ideal charged boson system," Annals of Physics, vol. 208, no. 1, pp. 149-215, 1991.

[197] A. S. Alexandrov and N. F. Mott, "Bipolarons," Reports on Progress in Physics, vol. 57, no. 12, pp. 1197-1288, 1994.

[198] R. Friedberg and T. D. Lee, "Comments on the superconductivity solution of an ideal charged boson system," Journal of Superconductivity and Novel Magnetism, vol. 19, no. 3-5, pp. 277-282, 2006.
[199] J. T. Devreese and A. S. Alexandrov, "Fröhlich polaron and bipolaron: recent developments," Reports on Progress in Physics, vol. 72, no. 6, Article ID 066501, 2009.

[200] T. M. Rice and L. Sneddon, "Real-space and k $\rightarrow$-space electron pairing in $\mathrm{BaPb}_{1-x} \mathrm{Bi}_{x} \mathrm{O}_{3}$," Physical Review Letters, vol. 47, no. 9, pp. 689-692, 1981.

[201] I. B. Bischofs, V. N. Kostur, and P. B. Allen, "Polaron and bipolaron defects in a charge density wave: a model for lightly doped $\mathrm{BaBiO}_{3}$," Physical Review B, vol. 65, no. 11, Article ID 115112, 7 pages, 2002.

[202] I. B. Bischofs, P. B. Allen, V. N. Kostur, and R. Bhargava, "Topological doping of a three-dimensional Peierls system: predicted structure of doped $\mathrm{BaBiO}_{3}$," Physical Review $B$, vol. 66, no. 17, Article ID 174108, 5 pages, 2002.

[203] M. Merz, N. Nücker, S. Schuppler, et al., "X-ray absorption of $\mathrm{Ba}_{1-x} \mathrm{~K}_{x} \mathrm{BiO}_{3}$ and $\mathrm{BaPb}_{1-y} \mathrm{Bi}_{y} \mathrm{O}_{3}$ : competition between bipolaronic and charge-density wave states," Europhysics Letters, vol. 72, no. 2, pp. 275-281, 2005.

[204] D. G. Hinks, B. Dabrowski, J. D. Jorgensen, et al., "Synthesis, structure and superconductivity in the $\mathrm{Ba}_{1-x} \mathrm{~K}_{x} \mathrm{BiO}_{3-y}$ system," Nature, vol. 333, no. 6176, pp. 836-838, 1988.

[205] A. I. Golovashkin, L. N. Zherikhina, G. V. Kuleshova, A. M. Tskhovrebov, and M. L. Norton, "Self-consistent spatially inhomogeneous state in HTSC $\mathrm{Ba}_{1-x} \mathrm{~K}_{x} \mathrm{BiO}_{3}$ single crystals," Pis'ma v Zhurnal Eksperimental'noi i Teoreticheskoi Fiziki, vol. 129, p. 684, 2006.

[206] G. Iadonisi, M. Chiofalo, V. Cataudella, and D. Ninno, "Phonon-plasmon cooperative effects in the dilute largebipolaron gas: a possible mechanism for high- $T_{c}$ superconductivity," Physical Review B, vol. 48, no. 17, pp. 1296612978, 1993.

[207] T. Nishio, J. Ahmad, and H. Uwe, "Spectroscopic observation of bipolaronic point defects in $\mathrm{Ba}_{1-x} \mathrm{~K}_{x} \mathrm{BiO}_{3}$," Physical Review Letters, vol. 95, no. 17, Article ID 176403, 4 pages, 2005.

[208] A. S. Alexandrov, " $d$-wave Bose-Einstein condensate and tunnelling in superconducting cuprates," Physica C, vol. 305, no. 1-2, pp. 46-56, 1998.

[209] A. S. Alexandrov and V. V. Kabanov, "Parameter-free expression for superconducting $T_{c}$ in cuprates," Physical Review B, vol. 59, no. 21, pp. 13628-13631, 1999.

[210] A. S. Alexandrov, "Polaron dynamics and bipolaron condensation in cuprates," Physical Review B, vol. 61, no. 18, pp. 12315-12327, 2000.

[211] Q. Chen, J. Stajic, S. Tan, and K. Levin, "BCS-BEC crossover: from high temperature superconductors to ultracold superfluids," Physics Reports, vol. 412, no. 1, pp. 1-88, 2005.

[212] L. S. Marchenko, D. P. Moiseev, E. A. Muzalevskii, and S. K. Uvarova, "Superconductivity in the solid-solutions $\mathrm{BaPb}_{1-x} \mathrm{Bi}_{x} \mathrm{O}_{3}$," Inorganic Materials, vol. 15, p. 1492, 1979.

[213] T. D. Thanh, A. Koma, and S. Tanaka, "Superconductivity in the $\mathrm{BaPb}_{1-x} \mathrm{Bi}_{x} \mathrm{O}_{3}$ system," Applied Physics, vol. 22, no. 2, pp. 205-212, 1980.

[214] D. P. Moiseev, S. K. Uvarova, and M. B. Fenik, "Hall voltage and conductivity in the superconducting oxide system $\mathrm{BaPbO}_{3}-\mathrm{BaBiO}_{3}$," Fizika Tverdogo Tela, vol. 23, p. 2347, 1981.

[215] M. L. Cohen, "Superconductivity in low-carrier-density systems: degenerate semiconductors," in Superconductivity, R. D. Parks, Ed., vol. 1, p. 615, Marcel Dekker, New York, NY, USA, 1969.

[216] Y. Takada, "Theory of superconductivity in polar semiconductors and its application to $n$-type semiconducting $\mathrm{SrTiO}_{3}$," Journal of the Physical Society of Japan, vol. 49, no. 4, pp. 1267-1275, 1980. 
[217] T. Egami, "Nano-scale complexities in the superconducting cuprates," Journal of Physics and Chemistry of Solids, vol. 69, no. 9, pp. 2191-2194, 2008.

[218] L. Pietronero and E. Cappelluti, "Superconductivity, nonadiabaticity and strong correlation in the light of recent experiments," Journal of Physics: Conference Series, vol. 108, no. 1, Article ID 012025, 2008.

[219] R. Friedberg and T. D. Lee, "Gap energy and long-range order in the boson-fermion model of superconductivity," Physical Review B, vol. 40, no. 10, pp. 6745-6762, 1989.

[220] J. Ranninger and J. M. Robin, "The boson-fermion model of high- $T_{c}$ superconductivity. Doping dependence," Physica $C$, vol. 253, no. 3-4, pp. 279-291, 1995.

[221] J. Ranninger and J. M. Robin, "Manifestations of the pseudogap in the boson-fermion model for Bose-Einsteincondensation-driven superconductivity," Physical Review B, vol. 53, no. 18, pp. R11961-R11963, 1996.

[222] E. Altman and A. Auerbach, "Plaquette boson-fermion model of cuprates," Physical Review B, vol. 65, no. 10, Article ID 104508, 15 pages, 2002.

[223] T. Domański and K. I. Wysokiński, "Effect of disorder on superconductivity in the boson-fermion model," Physical Review B, vol. 66, no. 6, Article ID 064517, 6 pages, 2002.

[224] R. Micnas, S. Robaszkiewicz, and A. Bussmann-Holder, "Anisotropic superconductivity in systems with coexisting electrons and local pairs," Physical Review B, vol. 66, no. 10, Article ID 104516, 9 pages, 2002.

[225] R. Micnas, "Superfluid transition temperature of the bosonfermion model on a lattice," Physical Review B, vol. 76, no. 18, Article ID 184507, 5 pages, 2007.

[226] A. S. Alexandrov, "Boson-fermion model beyond the meanfield approximation," Journal of Physics: Condensed Matter, vol. 8, no. 37, pp. 6923-6932, 1996.

[227] A. S. Alexandrov, "Self-energy catastrophe of the bosonfermion model of high-temperature superconductors," Physica C, vol. 316, no. 3, pp. 239-242, 1999.

[228] T. Egami, "Electron-phonon coupling in high- $T_{c}$ superconductors," in Superconductivity in Complex Systems, K. A. Müller and A. Bussmann-Holder, Eds., vol. 114 of Structure and Bonding, p. 267, Springer, Berlin, Germany, 2005.

[229] M. Fratini, N. Poccia, and A. Bianconi, "The Feshbach resonance and nanoscale phase separation in a polaron liquid near the quantum critical point for a polaron Wigner crystal," Journal of Physics: Conference Series, vol. 108, no. 1, Article ID 012036, 2008.

[230] A. R. Bishop, "HTC oxides: a collusion of spin, charge and lattice," Journal of Physics: Conference Series, vol. 108, no. 1, Article ID 012027, 2008.

[231] A. Bussmann-Holder and H. Keller, "Unconventional isotope effects, multi-component superconductivity and polaron formation in high temperature cuprate superconductors," Journal of Physics: Conference Series, vol. 108, no. 1, Article ID 012019, 2008.

[232] O. Gunnarsson and O. Rösch, "Interplay between electronphonon and Coulomb interactions in cuprates," Journal of Physics: Condensed Matter, vol. 20, no. 4, Article ID 043201, 2008.

[233] C. S. Ting, D. N. Talwar, and K. L. Ngai, "Possible mechanism of superconductivity in metal-semiconductor eutectic alloys," Physical Review Letters, vol. 45, no. 14, pp. 1213-1216, 1980.

[234] Y. Bar-Yam, "Two-component superconductivity. I. Introduction and phenomenology," Physical Review B, vol. 43, no. 1, pp. 359-377, 1991.
[235] Y. Bar-Yam, “Two-component superconductivity. II. Copper oxide high- $T_{c}$ superconductors," Physical Review B, vol. 43, no. 4, pp. 2601-2614, 1991.

[236] P. W. Anderson, "Model for the electronic structure of amorphous semiconductors," Physical Review Letters, vol. 34, no. 15, pp. 953-955, 1975.

[237] G. A. Levin and K. F. Quader, "Tunneling asymmetry in high$T_{c}$ cuprates: possible evidence for a submerged band with nondegenerate fermions," Physical Review B, vol. 48, no. 21, pp. 16184-16187, 1993.

[238] K. F. Quader and G. A. Levin, "Origin and consequences of the 'gap' in the cuprate normal state," Philosophical Magazine B, vol. 74, no. 5, pp. 611-632, 1996.

[239] H. Fröhlich, "Superconductivity in metals with incomplete inner shells," Journal of Physics C, vol. 1, no. 2, pp. 544-548, 1968.

[240] E. A. Pashitskii, "Plasmon mechanism of superconductivity in degenerate semiconductors and semimetals. I," Pis'ma $v$ Zhurnal Eksperimental'noi i Teoreticheskoi Fiziki, vol. 55, p. 2387, 1968.

[241] J. Ruvalds, "Are there acoustic plasmons?" Advances in Physics, vol. 30, no. 5, pp. 677-695, 1981.

[242] Y. Takada, "Plasmon mechanism of superconductivity in the multivalley electron gas," Journal of the Physical Society of Japan, vol. 61, pp. 238-253, 1992.

[243] R. Blank and H. Haug, "Theory of plasmon-assisted electron pairing in semiconductors," Physica Status Solidi B, vol. 188, no. 1, pp. 105-123, 1995.

[244] E. A. Pashitskii, "Low frequency charge density excitations and high- $T_{c}$ superconductivity in cuprate metal-oxide compounds. 1. The HTSC problem prior to a discovery of the high- $T_{c}$ superconductors: Predictions and premises," Fizika Nizkikh Temperatur, vol. 21, p. 995, 1995.

[245] E. A. Pashitskii, "Low frequency charge density excitations and high- $T_{c}$ superconductivity in cuprate metal-oxide compounds. 2. The HTSC problem after discovery of hightemperature superconductors: Achievements and outlooks," Fizika Nizkikh Temperatur, vol. 21, p. 1091, 1995.

[246] A. Bill, H. Morawitz, and V. Z. Kresin, "Electronic collective modes and superconductivity in layered conductors," Physical Review B, vol. 68, no. 14, Article ID 144519, 12 pages, 2003.

[247] E. A. Pashitskii and V. I. Pentegov, "On the plasmon mechanism of high- $T_{c}$ superconductivity in layered crystals and two-dimensional systems," Fizika Nizkikh Temperatur, vol. 34, no. 2, pp. 148-160, 2008.

[248] V. P. Antropov, O. Gunnarsson, and A. I. Liechtenstein, "Phonons, electron-phonon, and electron-plasmon coupling in $\mathrm{C}_{60}$ compounds," Physical Review B, vol. 48, no. 10, pp. 7651-7664, 1993.

[249] J. K. Hulm, M. Ashkin, D. W. Deis, and C. K. Jones, in Progress in Low Temperature Physics, vol. 6, p. 205, North-Holland, Amsterdam, The Netherlands, 1970.

[250] V. L. Ginzburg and D. A. Kirzhnitz, Eds., Problem of High-Temperature Superconductivity, Nauka, Moscow, Russia, 1977.

[251] V. L. Ginzburg and D. A. Kirzhnitz, Eds., High Temperature Superconductivity, Consultants Bureau, New York, NY, USA, 1982.

[252] S. A. Nemov and Yu. I. Ravich, "Thallium dopant in lead chalcogenides: investigation methods and peculiarities," Uspekhi Fizicheskikh Nauk, vol. 168, p. 817, 1998. 
[253] G. Binnig, A. Baratoff, H. E. Hoenig, and J. G. Bednorz, "Two-band superconductivity in $\mathrm{Nb}$-doped $\mathrm{SrTiO}_{3}$," Physical Review Letters, vol. 45, no. 16, pp. 1352-1355, 1980.

[254] V. A. Moskalenko, "Superconductivity of metals taking into account the overlap of the energy bands," Fizika Metallov $i$ Metallovedenie, vol. 8, p. 503, 1959.

[255] H. Suhl, B. T. Matthias, and L. R. Walker, "BardeenCooper-Schrieffer theory of superconductivity in the case of overlapping bands," Physical Review Letters, vol. 3, no. 12, pp. 552-554, 1959.

[256] A. M. Gabovich and E. A. Pashitskii, "Superconductivity of many-valley semiconductors," Ukrainskii Fizychnyi Zhurnal, vol. 20, p. 1814, 1975.

[257] A. L. Kasatkin and E. A. Pashitskii, "Josephson effect in twoband superconductors," Ukrainskii Fizychnyi Zhurnal, vol. 21, p. 578, 1976.

[258] L. N. Bulaevskii and M. V. Zyskin, "Energy gap in layered superconductors," Physical Review B, vol. 42, no. 16, pp. 10230-10240, 1990.

[259] V. Z. Kresin and S. A. Wolf, "Induced superconducting state and two-gap structure: application to cuprate superconductors and conventional multilayers," Physical Review B, vol. 46, no. 10, pp. 6458-6471, 1992.

[260] S. H. Liu and R. A. Klemm, "Energy gap structure and tunneling characteristics of layered superconductors," Physica C, vol. 216, no. 3-4, pp. 293-304, 1993.

[261] N. Klein, N. Tellmann, H. Schulz, K. Urban, S. A. Wolf, and V. Z. Kresin, "Evidence of two-gap s-wave superconductivity in $\mathrm{YBa}_{2} \mathrm{Cu}_{3} \mathrm{O}_{7-x}$ from microwave surface impedance measurements," Physical Review Letters, vol. 71, no. 20, pp. 33553358, 1993.

[262] A. Bussmann-Holder, L. Genzel, A. Simon, and A. R. Bishop, "Gap distribution and multigap-coupling in high $T_{c}$ 's," Zeitschrift für Physik B, vol. 92, no. 2, pp. 149-154, 1993.

[263] N. Kristoffel, P. Konsin, and T. Örd, "Two-band model for high-temperature superconductivity," La Rivista Del Nuovo Cimento, vol. 17, no. 9, pp. 1-41, 1994.

[264] P. Konsin and B. Sorkin, “Time-dependent Ginzburg-Landau equations for a two-band superconductor and the relaxation times of the order parameters in $\mathrm{Y}_{1-x} \mathrm{Ca}_{x} \mathrm{Ba}_{2} \mathrm{Cu}_{3} \mathrm{O}_{7-\delta}$," Physica Status Solidi B, vol. 244, no. 9, pp. 3254-3259, 2007.

[265] A. Bussmann-Holder, R. Khasanov, A. Shengelaya, et al., "Mixed order parameter symmetries in cuprate superconductors," Europhysics Letters, vol. 77, no. 2, Article ID 27002, 4 pages, 2007.

[266] O. V. Dolgov, I. I. Mazin, D. Parker, and A. A. Golubov, "Interband superconductivity: contrasts between BardeenCooper-Schrieffer and Eliashberg theories," Physical Review B, vol. 79, no. 6, Article ID 060502, 4 pages, 2009.

[267] A. Y. Liu, I. I. Mazin, and J. Kortus, "Beyond Eliashberg superconductivity in $\mathrm{MgB}_{2}$ : anharmonicity, two-phonon scattering, and multiple gaps," Physical Review Letters, vol. 87, no. 8, Article ID 087005, 4 pages, 2001.

[268] I. I. Mazin and V. P. Antropov, "Electronic structure, electron-phonon coupling, and multiband effects in $\mathrm{MgB}_{2}$," Physica C, vol. 385, no. 1-2, pp. 49-65, 2003.

[269] X. X. Xi, "MgB $\mathrm{Mg}_{2}$ thin films," Superconductor Science and Technology, vol. 22, no. 4, Article ID 043001, 15 pages, 2009.

[270] V. A. Gasparov, N. S. Sidorov, and I. I. Zver'Kova, "Twogap superconductivity in $\mathrm{ZrB}_{12}$ : temperature dependence of critical magnetic fields in single crystals," Physical Review B, vol. 73, no. 9, Article ID 094510, 11 pages, 2006.
[271] Yu. A. Nefyodov, A. M. Shuvaev, and M. R. Trunin, "Microwave response of $\mathrm{V}_{3} \mathrm{Si}$ single crystals: evidence for two-gap superconductivity," Europhysics Letters, vol. 72, no. 4, pp. 638-644, 2005.

[272] I. Bonalde, R. L. Ribeiro, W. Brämer-Escamilla, G. Mu, and H. H. Wen, "Possible two-gap behavior in noncentrosymmetric superconductor $\mathrm{Mg}_{10} \operatorname{Ir}_{19} \mathrm{~B}_{16}$ : a penetration depth study," Physical Review B, vol. 79, no. 5, Article ID 052506, 4 pages, 2009.

[273] C. L. Huang, J.-Y. Lin, C. P. Sun, et al., "Comparative analysis of specific heat of $\mathrm{YNi}_{2} \mathrm{~B}_{2} \mathrm{C}$ using nodal and two-gap models," Physical Review B, vol. 73, no. 1, Article ID 012502, 4 pages, 2006.

[274] C. L. Huang, J.-Y. Lin, Y. T. Chang, et al., "Experimental evidence for a two-gap structure of superconducting $\mathrm{NbSe}_{2}$ : a specific-heat study in external magnetic fields," Physical Review B, vol. 76, no. 21, Article ID 212504, 4 pages, 2007.

[275] M. D. Hossain, Z. Salman, D. Wang, et al., "Low-field cross spin relaxation of ${ }^{8} \mathrm{Li}$ in superconducting $\mathrm{NbSe}_{2}$," Physical Review B, vol. 79, no. 14, Article ID 144518, 6 pages, 2009.

[276] T. Tamegai, Y. Nakajima, T. Nakagawa, G. J. Li, and H. Harima, "Two-gap superconductivity in $\mathrm{R}_{2} \mathrm{Fe}_{3} \mathrm{Si}_{5}(R=\mathrm{Lu}$ and Sc)," Journal of Physics: Conference Series, vol. 150, Article ID 052264, 4 pages, 2009.

[277] Y. Nakajima, G. J. Li, and T. Tamegai, "Low-temperature specific-heat study of $\mathrm{Sc}_{5} \mathrm{Ir}_{4} \mathrm{Si}_{10}$," Journal of Physics: Conference Series, vol. 150, Article ID 052180, 4 pages, 2009.

[278] N. E. Phillips, N. Oeschler, R. A. Fisher, J. E. Gordon, M.L. Foo, and R. J. Cava, "Specific-heat of $\mathrm{Na}_{0.35} \mathrm{CoO}_{2} \cdot 1.3 \mathrm{H}_{2} \mathrm{O}$ : effects of sample age and pair breaking on two-gap superconductivity," Physica C, vol. 460-462, part 1, pp. 473-474, 2007.

[279] R. S. Gonnelli, M. Tortello, D. Daghero, G. A. Ummarino, V. A. Stepanov, and J. S. Kim, "Two-gap superconductivity in the Fe-1111 superconductor $\mathrm{LaFeAsO}_{1-x} \mathrm{~F}_{x}$ : a pointcontact Andreev-reflection study," Central European Journal of Physics, vol. 7, no. 2, pp. 251-256, 2009.

[280] P. Samuely, P. Szabó, Z. Pribulov, M. E. Tillman, S. L. Bud'Ko, and P. C. Canfield, "Possible two-gap superconductivity in $\mathrm{NdFeAsO}_{0.9} \mathrm{~F}_{0.1}$ probed by point-contact Andreev-reflection spectroscopy," Superconductor Science and Technology, vol. 22, no. 1, Article ID 014003, 6 pages, 2009.

[281] Y.-L. Wang, L. Shan, L. Fang, P. Cheng, C. Ren, and H.-H. Wen, "Multiple gaps in $\mathrm{SmFeAsO}_{0.9} \mathrm{~F}_{0.1}$ revealed by pointcontact spectroscopy," Superconductor Science and Technology, vol. 22, no. 1, Article ID 015018, 6 pages, 2009.

[282] P. Szabó, Z. Pribulová, G. Pristáš, S. L. Bud'ko, P. C. Canfield, and P. Samuely, "Evidence for two-gap superconductivity in $\mathrm{Ba}_{0.55} \mathrm{~K}_{0.45} \mathrm{Fe}_{2} \mathrm{As}_{2}$ from directional point-contact Andreevreflection spectroscopy," Physical Review B, vol. 79, no. 1, Article ID 012503, 4 pages, 2009.

[283] R. Khasanov, D. V. Evtushinsky, A. Amato, et al., "Two-gap superconductivity in $\mathrm{Ba}_{1-x} \mathrm{~K}_{x} \mathrm{Fe}_{2} \mathrm{As}_{2}$ : a complementary study of the magnetic penetration depth by muon-spin rotation and angle-resolved photoemission," Physical Review Letters, vol. 102, no. 18, Article ID 187005, 4 pages, 2009.

[284] A. I. Golovashkin and A. N. Lykov, "Gap peculiarities of the current-voltage characteristics of superconducting contacts with direct conductivity," Trudy Fiz. Inst. Akad. Nauk SSSR, vol. 190, p. 144, 1988.

[285] A. Sharoni, I. Felner, and O. Millo, “Tunneling spectroscopy and magnetization measurements of the superconducting properties of $\mathrm{MgB}_{2}$," Physical Review B, vol. 63, no. 22, Article ID 220508, 4 pages, 2001. 
[286] T. Takasaki, T. Ekino, T. Muranaka, H. Fujii, and J. Akimitsu, "Superconducting gap in polycrystalline $\mathrm{MgB}_{2}$ studied by electron tunneling," Physica C, vol. 378-381, part 1, pp. 229233, 2002.

[287] Yu. G. Naidyuk, I. K. Yanson, L. V. Tyutrina, et al., “ Superconducting energy gap distribution in c-axis oriented $\mathrm{MgB}_{2}$ thin film from point contact study," JETP Letters, vol. 75, no. 5, pp. 238-241, 2002.

[288] H. Schmidt, J. F. Zasadzinski, K. E. Gray, and D. G. Hinks, "Break-junction tunneling on $\mathrm{MgB}_{2}$," Physica $C$, vol. 385, no. 1-2, pp. 221-232, 2003.

[289] T. Ekino, T. Takasaki, T. Muranaka, J. Akimitsu, and H. Fujii, "Tunneling spectroscopy of the superconducting gap in $\mathrm{MgB}_{2}$," Physical Review B, vol. 67, no. 9, Article ID 094504, 2003.

[290] B. Barbiellini, O. Fischer, M. Peter, Ch. Renner, and M. Weger, "Gap distribution of the tunneling spectra in $\mathrm{Bi}_{2} \mathrm{Sr}_{2} \mathrm{CaCu}_{2} \mathrm{O}_{x}$ and some other superconductors," Physica C, vol. 220, no. 12, pp. 55-60, 1994.

[291] M. C. Boyer, W. D. Wise, K. Chatterjee, et al., "Imaging the two gaps of the high-temperature superconductor $\mathrm{Bi}_{2} \mathrm{Sr}_{2} \mathrm{CuO}_{6+x}$," Nature Physics, vol. 3, no. 11, pp. 802-806, 2007.

[292] C. E. Gough, M. S. Colclough, E. M. Forgan, et al., "Flux quantization in a high- $T_{c}$ superconductor," Nature, vol. 326, no. 6116 , p. $855,1987$.

[293] M. N. Keene, T. J. Jackson, and C. E. Gough, "Demonstration of the phase coherence of the superconducting wavefunctions between conventional and high- $T_{c}$ superconductors," Nature, vol. 340, no. 6230, pp. 210-211, 1989.

[294] E. Dagotto, "Correlated electrons in high-temperature superconductors," Reviews of Modern Physics, vol. 66, no. 3, pp. 763-840, 1994.

[295] A. P. Kampf, "Magnetic correlations in high temperature superconductivity," Physics Reports, vol. 249, no. 4-5, pp. 219-351, 1994.

[296] W. Brenig, "Aspects of electron correlations in the cuprate superconductors," Physics Reports, vol. 251, no. 3-4, pp. 153 266, 1995.

[297] M. Imada, A. Fujimori, and Y. Tokura, "Metal-insulator transitions," Reviews of Modern Physics, vol. 70, no. 4, pp. 1039-1263, 1998.

[298] S. Sachdev, "Colloquium: order and quantum phase transitions in the cuprate superconductors," Reviews of Modern Physics, vol. 75, no. 3, pp. 913-932, 2003.

[299] E. Demler, W. Hanke, and S.-C. Zhang, "SO(5) theory of antiferromagnetism and superconductivity," Reviews of Modern Physics, vol. 76, no. 3, pp. 909-974, 2004.

[300] T. Schneider, in The Physics of Superconductors. Vol. I: Conventional and High- $T_{c}$ Superconductors, K. H. Bennemann and J. B. Ketterson, Eds., p. 111, Springer, Berlin, Germany, 2004.

[301] T. Schneider and H. Keller, "Implications evinced by the phase diagram, anisotropy, magnetic penetration depths, isotope effects and conductivities of cuprate superconductors," New Journal of Physics, vol. 6, Article ID 144, 18 pages, 2004.

[302] P. A. Lee, N. Nagaosa, and X.-G. Wen, "Doping a Mott insulator: physics of high-temperature superconductivity," Reviews of Modern Physics, vol. 78, no. 1, Article ID 17, 69 pages, 2006.

[303] K. M. Shen and J. C. S. Davis, "Cuprate high- $T_{c}$ superconductors," Materials Today, vol. 11, no. 9, pp. 14-21, 2008.
[304] H. Alloul, J. Bobroff, M. Gabay, and P. J. Hirschfeld, "Defects in correlated metals and superconductors," Reviews of Modern Physics, vol. 81, no. 1, Article ID 45, 64 pages, 2009.

[305] D. J. Scalapino, "The case for $d_{x 2-y 2}$ pairing in the cuprate superconductors," Physics Reports, vol. 250, no. 6, pp. 329365, 1995.

[306] J. F. Annett, N. D. Goldenfeld, and A. J. Leggett, "Experimental constraints on the pairing state of the cuprate superconductors: an emerging consensus," in Physical Properties of High Temperature Superconductors V, D. M. Ginsberg, Ed., p. 375, World Scientific, River Ridge, NJ, USA, 1996.

[307] D. Pines, "Understanding high temperature superconductors: progress and prospects," Physica C, vol. 282-287, part 1, pp. 273-278, 1997.

[308] T. S. Nunner, J. Schmalian, and K. H. Bennemann, "Influence of electron-phonon interaction on spin-fluctuation-induced superconductivity," Physical Review B, vol. 59, no. 13, pp. 8859-8868, 1999.

[309] D. Manske, I. Eremin, and K. H. Bennemann, “Theory for electron-doped cuprate superconductors: $d$-wave symmetry order parameter," Physical Review B, vol. 62, no. 21, pp. 13922-13925, 2000.

[310] T. Dahm, V. Hinkov, S. V. Borisenko, et al., "Strength of the spin-fluctuation-mediated pairing interaction in a hightemperature superconductor," Nature Physics, vol. 5, no. 3, pp. 217-221, 2009.

[311] M. L. Cohen, "Electron-phonon-induced superconductivity," Journal of Superconductivity and Novel Magnetism, vol. 19, no. 3-5, pp. 283-290, 2006.

[312] G. Varelogiannis, "Phonon-mediated unconventional superconductivity in strongly correlated systems," Physical Review B, vol. 57, no. 21, pp. 13743-13764, 1998.

[313] G. Varelogiannis, "Small-q electron-phonon interaction and the phase diagram of the cuprates," Physica C, vol. 460-462, part 2, pp. 1125-1126, 2007.

[314] A. S. Alexandrov, " $d$-wave checkerboard bose condensate of mobile bipolarons," International Journal of Modern Physics $B$, vol. 21, no. 13-14, pp. 2301-2312, 2007.

[315] H. Kamimura and H. Ushio, "On the interplay of Jahn-Teller physics and Mott physics in cuprates," Journal of Physics: Conference Series, vol. 108, no. 1, Article ID 012030, 2008.

[316] A. S. Alexandrov, "Unconventional pairs glued by conventional phonons in cuprate superconductors," Journal of Superconductivity and Novel Magnetism, vol. 22, no. 2, pp. 103-107, 2009.

[317] E. A. Pashitskii and V. I. Pentegov, "The role of the charge density fluctuations and many-body Coulomb correlations in the mechanism of high-temperature superconductivity in cuprate metal-oxides," Fizika Nizkikh Temperatur, vol. 27, no. 2, pp. 140-152, 2001.

[318] H.-B. Schüttler and C.-H. Pao, "Isotope effect in $d$-wave superconductors," Physical Review Letters, vol. 75, no. 24, pp. 4504-4507, 1995.

[319] Yu. Bang, "Effects of phonon interaction on pairing in high$T_{c}$ superconductors," Physical Review B, vol. 78, no. 7, Article ID 075116, 2008.

[320] A. I. Lichtenstein and M. L. Kulić, "Electron-boson interaction can help d wave pairing self-consistent approach," Physica C, vol. 245, no. 1-2, pp. 186-192, 1995.

[321] T. Egami, P. Piekarz, and J.-H. Chung, "Role of phonons in the mechanism of high-temperature superconductivity," Physica C, vol. 408-410, no. 1-4, pp. 292-295, 2004. 
[322] E. G. Maksimov, "Electron-phonon interaction and superconductivity," Trudy Fiz. Inst. Akad. Nauk SSSR, vol. 86, p. 101, 1975.

[323] L. Tonks and I. Langmuir, "Oscillations in ionized gases," Physical Review, vol. 33, no. 2, pp. 195-210, 1929.

[324] D. Bohm and T. Staver, "Application of collective treatment of electron and ion vibrations to theories of conductivity and superconductivity ," Physical Review, vol. 84, no. 4, pp. 836837, 1951.

[325] V. P. Silin and A. A. Rukhadze, Electromagnetic Properties of Plasma and Plasma-Like Media, Gosatomizdat, Moscow, Russia, 1961.

[326] E. G. Brovman and Yu. M. Kagan, "Phonons in nontransition metals," Uspekhi Fizicheskikh Nauk, vol. 112, p. 369, 1974.

[327] B. T. Geilikman, "Adiabatic perturbation theory for metals and the problem of the lattice stability," Uspekhi Fizicheskikh Nauk, vol. 115, p. 403, 1975.

[328] M. A. Eggington and A. J. Leggett, "Is ODLRO a necessary condition for superfluidity?" Collective Phenomena, vol. 2, p. 81,1975

[329] B. T. Geilikman, Studies in Low Temperature Physics, Atomizdat, Moscow, Russia, 1979.

[330] V. M. Kontorovich, "Dynamical equations of the elasticity theory in metals," Uspekhi Fizicheskikh Nauk, vol. 142, p. 265, 1984.

[331] S. Yu. Savrasov and E. G. Maksimov, "Ab initio calculations of the lattice dynamics of crystals," Uspekhi Fizicheskikh Nauk, vol. 165, p. 773, 1995.

[332] E. G. Maksimov, D. Yu. Savrasov, and S. Yu. Savrasov, "Electron-phonon interaction and physical properies of metals," Uspekhi Fizicheskikh Nauk, vol. 167, p. 353, 1997.

[333] E. G. Maksimov and A. E. Karakozov, "On nonadiabatic effects in phonon spectra of metals," Uspekhi Fizicheskikh Nauk, vol. 178, p. 561, 2008.

[334] J. Lee, K. Fujita, K. McElroy, et al., "Interplay of electrong-lattice interactions and superconductivity in $\mathrm{Bi}_{2} \mathrm{Sr}_{2} \mathrm{CaCu}_{2} \mathrm{O}_{8+\delta}$," Nature, vol. 442, no. 7102, pp. 546-550, 2006.

[335] D. Reznik, L. Pintschovius, M. Ito, et al., "Electron-phonon coupling reflecting dynamic charge inhomogeneity in copper oxide superconductors," Nature, vol. 440, no. 7088, pp. 1170 1173, 2006.

[336] K. McElroy, J. Lee, J. A. Slezak, et al., "Atomic-scale sources and mechanism of nanoscale electronic disorder in $\mathrm{Bi}_{2} \mathrm{Sr}_{2} \mathrm{CaCu}_{2} \mathrm{O}_{8+\delta}$," Science, vol. 309, no. 5737, pp. 1048 $1052,2005$.

[337] D. Mihailovic and V. V. Kabanov, "Dynamic inhomogeneity, pairing and superconductivity in cuprates," in Superconductivity in Complex Systems, K. A. Muller and A. BussmannHolder, Eds., vol. 114 of Structure and Bonding, p. 331, Springer, Berlin, Germany, 2005.

[338] A. Sugimoto, S. Kashiwaya, H. Eisaki, et al., "Enhancement of electronic inhomogeneities due to out-of-plane disorder in $\mathrm{Bi}_{2} \mathrm{Sr}_{2} \mathrm{CuO}_{6+\delta}$ superconductors observed by scanning tunneling spectroscopy," Physical Review B, vol. 74, no. 9, Article ID 094503, 2006.

[339] A. Yazdani, "Visualizing pair formation on the atomic scale and the search for the mechanism of superconductivity in high- $T_{c}$ cuprates," Journal of Physics: Condensed Matter, vol. 21, no. 16, Article ID 164214, 2009.

[340] T. Kato, T. Machida, Y. Kamijo, R. Miyashita, and H. Sakata, "Spatial correlation between the LDOS modulation and electronic inhomogeneity in $\mathrm{Bi}_{2} \mathrm{Sr}_{2-x} \mathrm{La}_{x} \mathrm{CuO}_{6+\delta}$," Journal of
Physics: Conference Series, vol. 150, Article ID 052101, 4 pages, 2009.

[341] K. Kudo, T. Nishizaki, N. Okumura, and N. Kobayashi, "Electronic inhomogeneity in $\mathrm{Pb}$-substituted $\mathrm{Bi}_{2} \mathrm{Sr}_{2} \mathrm{CuO}_{6+\delta}$ studied by STM/STS measurements," Journal of Physics: Conference Series, vol. 150, Article ID 052133, 4 pages, 2009.

[342] M. Suzuki, T. Hamatani, Y. Yamada, K. Anagawa, and T. Watanabe, "Significantly doping-dependent Josephson critical current-inhomogeneity in real space or heterogeneity in k-space," Journal of Physics: Conference Series, vol. 150, Article ID 052252, 4 pages, 2009.

[343] W. D. Wise, K. Chatterjee, M. C. Boyer, et al., "Imaging nanoscale Fermi-surface variations in an inhomogeneous superconductor," Nature Physics, vol. 5, no. 3, pp. 213-216, 2009.

[344] W. D. Wise, M. C. Boyer, K. Chatterjee, et al., "Chargedensity-wave origin of cuprate checkerboard visualized by scanning tunnelling microscopy," Nature Physics, vol. 4, no. 9, pp. 696-699, 2008.

[345] X. M. Li, F. H. Li, H. Q. Luo, L. Fang, and H.-H. Wen, “Transmission electron microscopy study of one-dimensional incommensurate structural modulation in superconducting oxides $\mathrm{Bi}_{2+x} \mathrm{Sr}_{2-x} \mathrm{CuO}_{6+\delta}(0.10 \leq x \leq 0.40)$," Superconductor Science and Technology, vol. 22, no. 6, Article ID 065003, 2009.

[346] A. Mourachkine, High-Temperature Superconductivity in Cuprates, Kluwer Academic Publishers, Dordrecht, The Netherlands, 2002.

[347] J. F. Zasadzinski, “Tunneling spectroscopy of conventional and unconventional superconductors," in The Physics of Superconductors. Vol. 1: Conventional and High- $T_{c}$ Superconductors, K. H. Bennemann and J. B. Ketterson, Eds., p. 591, Springer, Berlin, Germany, 2003.

[348] $\varnothing$. Fischer, M. Kugler, I. Maggio-Aprile, C. Berthod, and C. Renner, "Scanning tunneling spectroscopy of hightemperature superconductors," Reviews of Modern Physics, vol. 79, no. 1, pp. 353-419, 2007.

[349] V. M. Krasnov, A. Yurgens, D. Winkler, P. Delsing, and T. Claeson, "Evidence for coexistence of the superconducting gap and the pseudogap in Bi-2212 from intrinsic tunneling spectroscopy," Physical Review Letters, vol. 84, no. 25, pp. 5860-5863, 2000.

[350] M. Oda, Y. H. Liu, T. Kurosawa, K. Takeyama, N. Momono, and M. Ido, "On the relations among the pseudogap, electronic charge order and Fermi-arc superconductivity in $\mathrm{Bi}_{2} \mathrm{Sr}_{2} \mathrm{CaCu}_{2} \mathrm{O}_{8+\delta}$," Journal of Physics: Conference Series, vol. 108, Article ID 012008, 6 pages, 2008.

[351] K. Tanaka, W. S. Lee, Z. Hussain, and Z. X. Shen, "Direct evidence of two gaps in underdoped Bi2212," Journal of Physics: Conference Series, vol. 108, no. 1, Article ID 012014, 2008.

[352] A. A. Kordyuk, S. V. Borisenko, V. B. Zabolotnyy, et al., "Nonmonotonic pseudogap in high- $T_{c}$ cuprates," Physical Review B, vol. 79, no. 2, Article ID 020504, 2009.

[353] W. S. Lee, I. M. Vishik, D. H. Lu, and Z.-X. Shen, "A brief update of angle-resolved photoemission spectroscopy on a correlated electron system," Journal of Physics: Condensed Matter, vol. 21, no. 16, Article ID 164217, 2009.

[354] R.-H. He, K. Tanaka, S.-K. Mo, et al., "Energy gaps in the failed high- $T_{c}$ superconductor $\mathrm{La}_{1.875} \mathrm{Ba}_{0.125} \mathrm{CuO}_{4}$," Nature Physics, vol. 5, no. 2, pp. 119-123, 2009.

[355] J. Meng, W. Zhang, G. Liu, et al., "Monotonic $d$-wave superconducting gap of the optimally doped $\mathrm{Bi}_{2} \mathrm{Sr}_{1.6} \mathrm{La}_{0.4} \mathrm{CuO}_{6}$ superconductor by laser-based angle-resolved photoemission 
spectroscopy," Physical Review B, vol. 79, no. 2, Article ID 024514, 2009.

[356] T. Kondo, R. Khasanov, T. Takeuchi, J. Schmalian, and A. Kaminski, "Competition between the pseudogap and superconductivity in the high- $T_{c}$ copper oxides," Nature, vol. 457, no. 7227, pp. 296-300, 2009.

[357] M. L. Teague, A. D. Beyer, M. S. Grinolds, S. I. Lee, and N.C. Yeh, "Observation of vortices and hidden pseudogap from scanning tunneling spectroscopic studies of the electrondoped cuprate superconductor $\mathrm{Sr}_{0.9} \mathrm{La}_{0.1} \mathrm{CuO}_{2}$," Europhysics Letters, vol. 85, no. 1, Article ID 17004, 2009.

[358] A. Sugimoto, K. Shohara, T. Ekino, et al., "Scanning tunneling microscopy/spectroscopy on multi-layered cuprate superconductor $\mathrm{Ba}_{2} \mathrm{Ca}_{5} \mathrm{Cu}_{6} \mathrm{O}_{12}\left(\mathrm{O}_{1-x} \mathrm{~F}_{x}\right)_{2}$," Physica $C$, vol. 469, no. 15-20, pp. 1020-1023, 2009.

[359] K. Mcelroy, D.-H. Lee, J. E. Hoffman, et al., "Coincidence of checkerboard charge order and antinodal state decoherence in strongly underdoped superconducting $\mathrm{Bi}_{2} \mathrm{Sr}_{2} \mathrm{CaCu}_{2} \mathrm{O}_{8+\delta}$," Physical Review Letters, vol. 94, no. 19, Article ID 197005, 2005.

[360] J. P. Castellan, B. D. Gaulin, H. A. Dabkowska, et al., "Two- and three-dimensional incommensurate modulation in optimally-doped $\mathrm{Bi}_{2} \mathrm{Sr}_{2} \mathrm{CaCu}_{2} \mathrm{O}_{8+\delta}$," Physical Review B, vol. 73, no. 17, Article ID 174505, 2006.

[361] J. Etrillard, P. Bourges, and C. T. Lin, "Incommensurate composite structure of the superconductor $\mathrm{Bi}_{2} \mathrm{Sr}_{2} \mathrm{CaCu}_{2} \mathrm{O}_{8+\delta}$," Physical Review B, vol. 62, no. 1, pp. 150-153, 2000.

[362] G. Kinoda, T. Hasegawa, S. Nakao, et al., "Electronic structures of two-phase microstructures $\alpha$ and $\beta$ in heavily $\mathrm{Pb}$ doped $\mathrm{Bi}_{2} \mathrm{Sr}_{2} \mathrm{CaCu}_{2} \mathrm{O}_{y}$ single crystals investigated by scanning tunneling microscopy/spectroscopy," Applied Physics Letters, vol. 83, no. 6, pp. 1178-1180, 2003.

[363] A. Sugimoto, S. Kashiwaya, H. Eisaki, et al., "Correlation between modulation structure and electronic inhomogeneity on $\mathrm{Pb}$-doped Bi-2212 single crystals," Physica C, vol. 426431, part 1, pp. 390-395, 2005.

[364] M. Vershinin, S. Misra, S. Ono, Y. Abe, Y. Ando, and A. Yazdani, "Local ordering in the pseudogap state of the high$T_{c}$ superconductor $\mathrm{Bi}_{2} \mathrm{Sr}_{2} \mathrm{CaCu}_{2} \mathrm{O}_{8+\delta}$," Science, vol. 303, no. 5666, pp. 1995-1998, 2004.

[365] A. Bianconi, M. Lusignoli, N. L. Saini, P. Bordet, A. Kvick, and P. G. Radaelli, "Stripe structure of the $\mathrm{CuO}_{2}$ plane in $\mathrm{Bi}_{2} \mathrm{Sr}_{2} \mathrm{CaCu}_{2} \mathrm{O}_{8+y}$ by anomalous X-ray diffraction," Physical Review B, vol. 54, no. 6, pp. 4310-4314, 1996.

[366] E. V. L. de Mello and E. S. Caixeiro, "The theory of local superconductivity and phase separation applied to cuprates," Journal of Superconductivity, vol. 18, no. 5-6, pp. 53-57, 2005.

[367] V. Z. Kresin, Yu. N. Ovchinnikov, and S. A. Wolf, "Inhomogeneous superconductivity and the "pseudogap" state of novel superconductors," Physics Reports, vol. 431, no. 5, pp. 231259, 2006.

[368] J. E. Hoffman, E. W. Hudson, K. M. Lang, et al., "A four unit cell periodic pattern of quasi-particle states surrounding vortex cores in $\mathrm{Bi}_{2} \mathrm{Sr}_{2} \mathrm{CaCu}_{2} \mathrm{O}_{8+\delta}$," Science, vol. 295, no. 5554, pp. 466-469, 2002.

[369] N. Momono, A. Hashimoto, Y. Kobatake, S. Nakamura, M. Oda, and M. Ido, "STM/STS study on local electronic states of underdoped $\mathrm{Bi}_{2} \mathrm{Sr}_{2} \mathrm{CaCu}_{2} \mathrm{O}_{8+\delta}$," International Journal of Modern Physics B, vol. 19, no. 1-3, pp. 231-234, 2005.

[370] A. Hashimoto, N. Momono, M. Oda, and M. Ido, "Scanning tunneling microscopy and spectroscopy study of $4 a \times 4 a$ electronic charge order and the inhomogeneous pairing gap in superconducting $\mathrm{Bi}_{2} \mathrm{Sr}_{2} \mathrm{CaCu}_{2} \mathrm{O}_{8+\delta}$," Physical Review B, vol. 74, no. 6, Article ID 064508, 2006.
[371] K. M. Shen, F. Ronning, D. H. Lu, et al., "Nodal quasiparticles and antinodal charge ordering in $\mathrm{Ca}_{2-x} \mathrm{Na}_{x} \mathrm{CuO}_{2} \mathrm{Cl}_{2}$," Science, vol. 307, no. 5711, pp. 901-904, 2005.

[372] J. A. Robertson, S. A. Kivelson, E. Fradkin, A. C. Fang, and A. Kapitulnik, "Distinguishing patterns of charge order: stripes or checkerboards," Physical Review B, vol. 74, no. 13, Article ID 134507, 2006.

[373] W. Meevasana, N. J. C. Ingle, D. H. Lu, et al., "Doping dependence of the coupling of electrons to bosonic modes in the single-layer high-temperature $\mathrm{Bi}_{2} \mathrm{Sr}_{2} \mathrm{CaCuO}_{6}$ superconductor," Physical Review Letters, vol. 96, no. 15, Article ID 157003, 2006.

[374] D. S. Inosov, V. B. Zabolotnyy, D. V. Evtushinsky, et al., "Fermi surface nesting in several transition metal dichalcogenides," New Journal of Physics, vol. 10, Article ID 125027, 2008.

[375] S. V. Borisenko, A. A. Kordyuk, V. B. Zabolotnyy, et al., "Two energy gaps and fermi-surface "arcs" in $\mathrm{NbSe}_{2}$," Physical Review Letters, vol. 102, no. 16, Article ID 166402, 2009.

[376] D. S. Inosov, D. V. Evtushinsky, V. B. Zabolotnyy, et al., "Temperature-dependent Fermi surface of $2 \mathrm{H}$ - $\mathrm{TaSe}_{2}$ driven by competing density wave order fluctuations," Physical Review B, vol. 79, no. 12, Article ID 125112, 2009.

[377] A. M. Gabovich, V. A. Medvedev, D. P. Moiseev, et al., "Superconductivity and thermal anomalies in the properties of the crystal lattice of the metal oxide $\mathrm{La}_{1.8} \mathrm{Ba}_{0.2} \mathrm{CuO}_{4}$, " Fizika Nizkikh Temperatur, vol. 13, no. 8, pp. 844-847, 1987.

[378] R. A. Klemm, "Striking similarities between the pseudogap phenomena in cuprates and in layered organic and dichalcogenide superconductors," Physica C, vol. 341-348, pp. 839$842,2000$.

[379] R. A. Klemm, "Origin of the pseudogap in high temperature superconductors," in Nonequilibrium Physics at Short Time Scales: Formation of Correlations, K. Morawetz, Ed., p. 381, Springer, Berlin, Germany, 2004.

[380] E. Morosan, H. W. Zandbergen, B. S. Dennis, et al., "Superconductivity in $\mathrm{Cu}_{x} \mathrm{TiSe}_{2}$," Nature Physics, vol. 2, no. 8, pp. 544-550, 2006.

[381] S. L. Bud'ko, P. C. Canfield, E. Morosan, R. J. Cava, and G. M. Schmiedeshoff, "Thermal expansion and effect of pressure on superconductivity in $\mathrm{Cu}_{x} \mathrm{TiSe}_{2}$," Journal of Physics: Condensed Matter, vol. 19, Article ID 176230, 8 pages, 2005.

[382] M. V. Kartsovnik, D. Andres, W. Biberacher, P. D. Grigoriev, E. A. Schuberth, and H. Müller, "New electronic phase transitions in $\alpha$-(BEDT-TTF ${ }_{2} \mathrm{KHg}(\mathrm{SCN})_{4}$," Journal de Physique IV, vol. 114, pp. 191-197, 2004.

[383] D. Graf, J. S. Brooks, M. Almeida, et al., "Evolution of superconductivity from a charge-density-wave ground state in pressurized $(\mathrm{Per})_{2}\left[\mathrm{Au}(\mathrm{mnt})_{2}\right]$," Europhysics Letters, vol. 85, no. 2, Article ID 27009, 2009.

[384] J. J. Hamlin, D. A. Zocco, T. A. Sayles, M. B. Maple, J.-H. Chu, and I. R. Fisher, "Pressure-induced superconducting phase in the charge-density-wave compound terbium tritelluride," Physical Review Letters, vol. 102, no. 17, Article ID 177002, 2009.

[385] G. Bilbro and W. L. McMillan, "Theoretical model of superconductivity and the martensitic transformation in A15 compounds," Physical Review B, vol. 14, no. 5, pp. 1887-1892, 1976.

[386] A. M. Gabovich, M. S. Li, H. Szymczak, and A. I. Voitenko, "Thermodynamics of superconductors with charge-density waves," Journal of Physics: Condensed Matter, vol. 15, no. 17, pp. 2745-2753, 2003. 
[387] N. Oeschler, R. A. Fisher, N. E. Phillips, J. E. Gordon, M.-L. Foo, and R. J. Cava, "Specific heat of $\mathrm{Na}_{0.3} \mathrm{CoO}_{2} \cdot 1.3 \mathrm{H}_{2} \mathrm{O}$ : two energy gaps, nonmagnetic pair breaking, strong fluctuations in the superconducting state, and effects of sample age," Physical Review B, vol. 78, no. 5, Article ID 054528, 2008.

[388] H. Fu, N. Oeschler, R. A. Fisher, et al., "Competition between superconductivity and charge-density wave order in $\mathrm{Na}_{0.3} \mathrm{CoO}_{2} \cdot 1.3 \mathrm{H}_{2} \mathrm{O}$," Journal of Superconductivity and Novel Magnetism, vol. 22, no. 3, pp. 295-298, 2009.

[389] N. E. Phillips, R. A. Fisher, H. Fu, et al., "Superconductivity and charge-density-wave order in $\mathrm{Na}_{0.3} \mathrm{CoO}_{2} \cdot 1.3 \mathrm{H}_{2} \mathrm{O}$," Journal of Physics: Conference Series, vol. 150, Article ID 052210, 2009.

[390] M. S. Li, "Paramagnetic Meissner effect and related dynamical phenomena," Physics Reports, vol. 376, no. 3, pp. 133-223, 2003.

[391] G. K. Perkins, Y. Bugoslavsky, A. D. Caplin, et al., "Effects of proton irradiation and ageing on the superconducting properties of single crystalline and polycrystalline $\mathrm{MgB}_{2}$," Superconductor Science and Technology, vol. 17, no. 1, pp. 232-235, 2004.

[392] A. M. Gabovich, D. P. Moiseev, L. V. Prokopovich, S. K. Uvarova, and V. E. Yachmenev, "Experimental proof of bulk superconductivity in perovskite system $\mathrm{BaPb}_{1-x} \mathrm{Bi}_{x} \mathrm{O}_{3}$," Zhurnal Éksperimental'noi i Teoreticheskoi Fiziki, vol. 86, p. 1727, 1984.

[393] I. R. Gomersall and B. L. Gyorffy, "On the connection between high superconducting transition temperature, $T_{c}$, and lattice instability in transition metal compounds: $\mathrm{NbN}$," Journal of Physics F, vol. 3, pp. L138-L144, 1973.

[394] J. C. Phillips, "Lattice instabilities and high-temperature superconductivity," Physical Review Letters, vol. 26, no. 10, pp. 543-546, 1971.

[395] I. Nakayama, "Structural transformation and superconductivity in A-15 compounds," Journal of the Physical Society of Japan, vol. 43, p. 1533, 1977.

[396] A. M. Gabovich, D. P. Moiseev, and A. S. Shpigel, "The nature of superconductivity for solid solutions $\mathrm{BaPb}_{1-x} \mathrm{Bi}_{x} \mathrm{O}_{3}$ with a perovskite structure. Role of the electron spectrum dielectrization," Zhurnal Éksperimental'noi $i$ Teoreticheskoi Fiziki, vol. 83, p. 1383, 1982.

[397] A. M. Gabovich and A. S. Shpigel, "Thermodynamics of superconductors with charge and spin-density waves," Journal of Physics F, vol. 14, no. 12, pp. 3031-3039, 1984.

[398] A. M. Gabovich, "Partial dielectrization model for oxide superconductivity," in High- $T_{c}$ Superconductivity, Experiment and Theory, A. S. Davydov and V. M. Loktev, Eds., pp. 161169, Springer, Berlin, Germany, 1992.

[399] T. Ekino, Y. Sezaki, and H. Fujii, "Features of the energy gap above $T_{c}$ in $\mathrm{Bi}_{2} \mathrm{Sr}_{2} \mathrm{CaCu}_{2} \mathrm{O}_{8+\delta}$ as seen by break-junction tunneling," Physical Review B, vol. 60, no. 9, pp. 6916-6922, 1999.

[400] J. Demsar, R. Hudej, J. Karpinski, V. V. Kabanov, and D. Mihailovic, "Quasiparticle dynamics and gap structure in $\mathrm{HgBa}_{2} \mathrm{Ca}_{2} \mathrm{Cu}_{3} \mathrm{O}_{8+\delta}$ investigated with femtosecond spectroscopy," Physical Review B, vol. 63, no. 5, Article ID 054519, 7 pages, 2001.

[401] M. Le Tacon, A. Sacuto, A. Georges, et al., "Two energy scales and two distinct quasiparticle dynamics in the superconducting state of underdoped cuprates," Nature Physics, vol. 2, no. 8, pp. 537-543, 2006.

[402] K. Tanaka, W. S. Lee, D. H. Lu, et al., "Distinct fermimomentum-dependent energy gaps in deeply underdroped Bi2212," Science, vol. 314, no. 5807, pp. 1910-1913, 2006.
[403] W. S. Lee, I. M. Vishik, K. Tanaka, et al., "Abrupt onset of a second energy gap at the superconducting transition of underdoped Bi2212," Nature, vol. 450, no. 7166, pp. 81-84, 2007.

[404] T. Das, R. S. Markiewicz, and A. Bansil, "Competing order scenario of two-gap behavior in hole-doped cuprates," Physical Review B, vol. 77, no. 13, Article ID 134516, 2008.

[405] V. J. Emery and S. A. Klvelson, "Importance of phase fluctuations in superconductors with small superfluid density," Nature, vol. 374, no. 6521, pp. 434-437, 1995.

[406] M. R. Norman, D. Pines, and C. Kallin, "The pseudogap: friend or foe of high $T_{c}$ ?" Advances in Physics, vol. 54, no. 8, pp. 715-733, 2005.

[407] T. Valla, A. V. Fedorov, J. Lee, J. C. Davis, and G. D. Gu, "The ground state of the pseudogap in cuprate supercondustors," Science, vol. 314, no. 5807, pp. 1914-1916, 2006.

[408] A. Kanigel, U. Chatterjee, M. Randeria, et al., "Protected nodes and the collapse of fermi arcs in high- $T_{c}$ cuprate superconductors," Physical Review Letters, vol. 99, no. 15, Article ID 157001, 2007.

[409] A. Kanigel, U. Chatterjee, M. Randeria, et al., "Evidence for pairing above the transition temperature of cuprate superconductors from the electronic dispersion in the pseudogap phase," Physical Review Letters, vol. 101, no. 13, Article ID 137002, 2008.

[410] H.-B. Yang, J. D. Rameau, P. D. Johnson, T. Valla, A. Tsvelik, and G. D. Gu, "Emergence of preformed cooper pairs from the doped Mott insulating state in $\mathrm{Bi}_{2} \mathrm{Sr}_{2} \mathrm{CaCu}_{2} \mathrm{O}_{8+\delta}$, "Nature, vol. 456, no. 7218, pp. 77-80, 2008.

[411] M. Shi, J. Chang, S. Pailhés, et al., "Coherent $d$-Wave superconducting gap in underdoped $\mathrm{La}_{2-x} \mathrm{Sr}_{x} \mathrm{CuO}_{4}$ by angleresolved photoemission spectroscopy," Physical Review Letters, vol. 101, no. 4, Article ID 047002, 2008.

[412] S. Hüfner and F. Müller, "Temperature dependence of the gaps of high-temperature superconductors in the Fermi-arc region," Physical Review B, vol. 78, no. 1, Article ID 014521, 2008.

[413] S. Hüfner, M. A. Hossain, A. Damascelli, and G. A. Sawatzky, "Two gaps make a high-temperature superconductor?" Reports on Progress in Physics, vol. 71, no. 6, Article ID 062501, 2008.

[414] K. Nakayama, T. Sato, Y. Sekiba, et al., "Evolution of a pairing-induced pseudogap from the superconducting gap of $(\mathrm{Bi}, \mathrm{Pb})_{2} \mathrm{Sr}_{2} \mathrm{CuO}_{6}$," Physical Review Letters, vol. 102, no. 22, Article ID 227006, 2009.

[415] C.-C. Chien, Y. He, Q. Chen, and K. Levin, “Two-energy-gap preformed-pair scenario for cuprate superconductors: implications for angle-resolved photoemission spectroscopy," Physical Review B, vol. 79, no. 21, Article ID 214527, 2009.

[416] J. Lee, K. Fujita, A. R. Schmidt, et al., "Spectroscopic fingerprint of phase-incoherent superconductivity in the underdoped $\mathrm{Bi}_{2} \mathrm{Sr}_{2} \mathrm{CaCu}_{2} \mathrm{O}_{8+\delta}$," Science, vol. 325, no. 5944, pp. 1099-1103, 2009.

[417] V. M. Krasnov, "Interlayer tunneling spectroscopy of $\mathrm{Bi}_{2} \mathrm{Sr}_{2} \mathrm{CaCu}_{2} \mathrm{O}_{8+\delta}$ : a look from inside on the doping phase diagram of high- $T_{c}$ superconductors," Physical Review B, vol. 65, no. 14, Article ID 140504, 4 pages, 2002.

[418] E. E. M. Chia, J.-X. Zhu, D. Talbayev, et al., "Observation of competing order in a high- $T_{c}$ superconductor using femtosecond optical pulses," Physical Review Letters, vol. 99, no. 14, Article ID 147008, 2007.

[419] M. Hashimoto, T. Yoshida, A. Fujimori, et al., "Effects of out-of-plane disorder on the nodal quasiparticle and superconducting gap in single-layer $\mathrm{Bi}_{2} \mathrm{Sr}_{1.6} \mathrm{~L}_{0.4} \mathrm{CuO}_{6+\delta}$ 
( $L=$ La,Nd,Gd)," Physical Review B, vol. 79, no. 14, Article ID 144517, 2009.

[420] V. M. Krasnov, A. E. Kovalev, A. Yurgens, and D. Winkler, "Magnetic field dependence of the superconducting gap and the pseudogap in Bi2212 and $\mathrm{HgBr}_{2}$-Bi2212, studied by intrinsic tunneling spectroscopy," Physical Review Letters, vol. 86, no. 12, pp. 2657-2660, 2001.

[421] D. E. Moncton, J. D. Axe, and F. J. DiSalvo, "Neutron scattering study of the charge-density wave transitions in $2 \mathrm{H}-\mathrm{TaSe}_{2}$ and $2 \mathrm{H}-\mathrm{NbSe}_{2}, "$ Physical Review B, vol. 16, no. 2, pp. 801-819, 1977.

[422] K. Machida, "Spin density wave and superconductivity in highly anisotropic materials," Journal of the Physical Society of Japan, vol. 50, pp. 2195-2202, 1981.

[423] K. Machida and T. Matsubara, "Spin density wave and superconductivity in highly anisotropic materials. II. Detailed study of phase transitions," Journal of the Physical Society of Japan, vol. 50, p. 3231, 1981.

[424] K. Machida, "Spin density wave and superconductivity in highly anisotropic materials. III. Energy gap structure and non-magnetic impurity effects," Journal of the Physical Society of Japan, vol. 51, p. 1420, 1982.

[425] A. M. Gabovich, D. P. Moiseev, and A. S. Shpigel, "Thermodynamic properties of superconducting ceramics $\mathrm{BaPb}_{1-x} \mathrm{Bi}_{x} \mathrm{O}_{3}$," Journal of Physics $C$, vol. 15, no. 18, pp. L569L572, 1982.

[426] A. M. Gabovich and A. S. Shpigel, "Influence of impurity scattering on the critical temperature of superconductors with a partial gap in the electron spectrum," Journal of Low Temperature Physics, vol. 51, no. 5-6, pp. 581-599, 1983.

[427] A. M. Gabovich, A. S. Gerber, and A. S. Shpigel, "Thermodynamics of superconductors with charge- and spin-density waves," Physica Status Solidi B, vol. 141, no. 2, pp. 575-587, 1987.

[428] A. M. Gabovich and A. S. Shpigel, "Upper critical magnetic field of superconductors with a dielectric gap on the Fermisurface sections," Physical Review B, vol. 38, no. 1, pp. 297306, 1988.

[429] K. Levin, D. L. Mills, and S. L. Cunningham, "Incompatibility of BCS pairing and the Peierls distortion in one-dimensional systems. I. Mean-field theory," Physical Review B, vol. 10, no. 9, pp. 3821-3831, 1974.

[430] K. Levin, S. L. Cunningham, and D. L. Mills, "Incompatibility of BCS pairing and the Peierls distortion in one-dimensional systems. II. Fluctuation effects," Physical Review B, vol. 10, no. 9, pp. 3832-3843, 1974.

[431] Yu. V. Kopaev, "About the interplay theory between electron and structural transformations and superconductivity," Trudy Fiz. Inst. Akad. Nauk SSSR, vol. 86, p. 3, 1975.

[432] C. A. Balseiro and L. M. Falicov, "Superconductivity and charge-density waves," Physical Review B, vol. 20, no. 11, pp. 4457-4464, 1979.

[433] A. M. Gabovich and E. A. Pashitskii, "About the existence of mixed phase superconductor-excitonic-insulator in intrinsic semimetals," Fizika Tverdogo Tela, vol. 17, p. 1584, 1975.

[434] Y. Ishida, T. Shimojima, K. Ishizaka, et al., "Temperaturedependent pseudogap in the oxypnictides $\mathrm{LaFeAsO}_{1-x} \mathrm{~F}_{x}$ and $\mathrm{LaFePO}_{1-x} \mathrm{~F}_{x}$ seen via angle-integrated photoemission,” Physical Review B, vol. 79, no. 6, Article ID 060503, 2009.

[435] T. Mertelj, V. V. Kabanov, C. Gadermaier, et al., "Distinct pseudogap and quasiparticle relaxation dynamics in the superconducting state of nearly optimally doped $\mathrm{SmFeAsO}_{0.8} \mathrm{~F}_{0.2}$ single crystals," Physical Review Letters, vol. 102, no. 11, Article ID 117002, 2009.
[436] V. B. Zabolotnyy, D. S. Inosov, D. V. Evtushinsky, et al., " $(\pi, \pi)$ electronic order in iron arsenide superconductors," Nature, vol. 457, no. 7229, pp. 569-572, 2009.

[437] R. H. Friend and D. Jérome, "Periodic lattice distortions and charge density waves in one- and two-dimensional metals," Journal of Physics C, vol. 12, no. 8, pp. 1441-1477, 1979.

[438] P. B. Littlewood and V. Heine, "The effect of electron-electron interactions on the Peierls transition in metals with strong nesting of Fermi surfaces," Journal of Physics C, vol. 14, no. 21, pp. 2943-2949, 1981.

[439] M. Rotter, M. Tegel, D. Johrendt, I. Schellenberg, W. Hermes, and R. Pöttgen, "Spin-density-wave anomaly at $140 \mathrm{~K}$ in the ternary iron arsenide $\mathrm{BaFe}_{2} \mathrm{As}_{2}$," Physical Review B, vol. 78, no. 2, Article ID 020503, 2008.

[440] N. Miyakawa, K. Tokiwa, S. Mikusu, et al., "Tunneling studies of multilayered superconducting cuprate $\mathrm{Ba}_{2} \mathrm{Ca}_{3} \mathrm{Cu}_{4} \mathrm{O}_{12+\delta}$," International Journal of Modern Physics B, vol. 17, no. 18-20, pp. 3612-3616, 2003.

[441] K. K. Gomes, A. N. Pasupathy, A. Pushp, S. Ono, Y. Ando, and A. Yazdani, "Visualizing pair formation on the atomic scale in the high- $T_{c}$ superconductor $\mathrm{Bi}_{2} \mathrm{Sr}_{2} \mathrm{CaCu}_{2} \mathrm{O}_{8+\delta}$," Nature, vol. 447, no. 7144, pp. 569-572, 2007.

[442] N. Miyakawa, K. Tokiwa, S. Mikusu, et al., "Tunneling spectroscopy of trilayer high- $T_{c}$ cuprate, $\mathrm{TlBa}_{2} \mathrm{Ca}_{2} \mathrm{Cu}_{2} \mathrm{O}_{10+\delta}$," International Journal of Modern Physics B, vol. 19, no. 1-3, pp. 225-229, 2005.

[443] Y. DeWilde, N. Miyakawa, P. Guptasarma, et al., "Unusual strong-coupling effects in the tunneling spectroscopy of optimally doped and overdoped $\mathrm{Bi}_{2} \mathrm{Sr}_{2} \mathrm{CaCu}_{2} \mathrm{O}_{8+\delta}$," Physical Review Letters, vol. 80, no. 1, pp. 153-156, 1998.

[444] M. Eschrig and M. R. Norman, "Neutron resonance: modeling photoemission and tunneling data in the superconducting state of $\mathrm{Bi}_{2} \mathrm{Sr}_{2} \mathrm{CaCu}_{2} \mathrm{O}_{8+\delta}$," Physical Review Letters, vol. 85, no. 15, pp. 3261-3264, 2000.

[445] J.-X. Zhu, J. Sun, Q. Si, and A. V. Balatsky, "Effects of a collective spin resonance mode on the scanning tunneling microscopy spectra of $d$-wave superconductors," Physical Review Letters, vol. 92, no. 1, Article ID 017002, 4 pages, 2004.

[446] J. F. Zasadzinski, L. Ozyuzer, L. Coffey, K. E. Gray, D. G. Hinks, and C. Kendziora, "Persistence of strong electron coupling to a narrow boson spectrum in overdoped $\mathrm{Bi}_{2} \mathrm{Sr}_{2} \mathrm{CaCu}_{2} \mathrm{O}_{8+\delta}$ tunneling data," Physical Review Letters, vol. 96, no. 1, Article ID 017004, 2006.

[447] J.-X. Zhu, A. V. Balatsky, T. P. Devereaux, et al., "Fouriertransformed local density of states and tunneling into a $d$ wave superconductor with bosonic modes," Physical Review $B$, vol. 73, no. 1, Article ID 014511, 9 pages, 2006.

[448] P. W. Anderson and N. P. Ong, "Theory of asymmetric tunneling in the cuprate superconductors," Journal of Physics and Chemistry of Solids, vol. 67, no. 1-3, pp. 1-5, 2006.

[449] G. L. de Castro, C. Berthod, A. Piriou, E. Giannini, and $\varnothing$. Fischer, "Preeminent role of the van Hove singularity in the strong-coupling analysis of scanning tunneling spectroscopy for two-dimensional cuprate superconductors," Physical Review Letters, vol. 101, no. 26, Article ID 267004, 2008.

[450] J. Nieminen, H. Lin, R. S. Markiewicz, and A. Bansil, "Origin of the electron-hole asymmetry in the scanning tunneling spectrum of the high-temperature $\mathrm{Bi}_{2} \mathrm{Sr}_{2} \mathrm{CaCu}_{2} \mathrm{O}_{8+\delta}$ superconductor," Physical Review Letters, vol. 102, no. 3, Article ID 037001, 2009.

[451] J.-X. Liu, J.-C. Wan, A. M. Goldman, Y. C. Chang, and P. Z. Jiang, "Features of the density of states of high$T_{c}$ superconductors probed by vacuum tunneling," Physical Review Letters, vol. 67, no. 16, pp. 2195-2198, 1991. 
[452] T. Cren, D. Roditchev, W. Sacks, and J. Klein, "Nanometer scale mapping of the density of states in an inhomogeneous superconductor," Europhysics Letters, vol. 54, no. 1, pp. 8490, 2001.

[453] S. H. Pan, J. P. O’Neal, R. L. Badzey, et al., "Microscopic electronic inhomogeneity in the high- $T_{c}$ superconductor $\mathrm{Bi}_{2} \mathrm{Sr}_{2} \mathrm{CaCu}_{2} \mathrm{O}_{8+x}$," Nature, vol. 413, no. 6853, pp. 282-285, 2001.

[454] K. M. Lang, V. Madhavan, J. E. Hoffman, et al., "Imaging the granular structure of high- $T_{c}$ superconductivity in underdoped $\mathrm{Bi}_{2} \mathrm{Sr}_{2} \mathrm{CaCu}_{2} \mathrm{O}_{8+\delta}$," Nature, vol. 415, no. 6870, pp. 412-416, 2002.

[455] G. Kinoda, S. Nakao, T. Motohashi, et al., "Inhomogeneous electronic structures in heavily $\mathrm{Pb}$-doped $\mathrm{Bi}_{2} \mathrm{Sr}_{2} \mathrm{CaCu}_{2} \mathrm{O}_{y}$ single crystals probed by low temperature STM/STS," Physica C, vol. 388-389, pp. 273-274, 2003.

[456] H. Mashima, N. Fukuo, Y. Matsumoto, et al., "Electronic inhomogeneity of heavily overdoped $\mathrm{Bi}_{2-\mathrm{x}} \mathrm{Pb}_{\mathrm{x}} \mathrm{Sr}_{2} \mathrm{CuO}_{y}$ studied by low-temperature scanning tunneling microscopy/spectroscopy," Physical Review B, vol. 73, no. 6, Article ID 060502, 4 pages, 2006.

[457] A. C. Fang, L. Capriotti, D. J. Scalapino, et al., "Gapinhomogeneity-induced electronic states in superconducting $\mathrm{Bi}_{2} \mathrm{Sr}_{2} \mathrm{CaCu}_{2} \mathrm{O}_{8+\delta}$," Physical Review Letters, vol. 96, no. 1, Article ID 017007, 2006.

[458] J. A. Slezak, J. Lee, M. Wang, et al., "Imaging the impact on cuprate superconductivity of varying the interatomic distances within individual crystal unit cells," Proceedings of the National Academy of Sciences of the United States of America, vol. 105, no. 9, pp. 3203-3208, 2008.

[459] K. Fujita, J. Lee, M. Wang, et al., "Imaging the effect of electron lattice interactions on high- $T_{c}$ superconductivity in $\mathrm{Bi}_{2} \mathrm{Sr}_{2} \mathrm{CaCu}_{2} \mathrm{O}_{8+\delta}$," Journal of Physics: Conference Series, vol. 108, no. 1, Article ID 012028, 2008.

[460] K. Anagawa, T. Watanabe, and M. Suzuki, "Superconducting gap and pseudogap for overdoped $\mathrm{Bi}_{2-x} \mathrm{~Pb}_{x} \mathrm{Sr}_{2} \mathrm{CaCu}_{2} \mathrm{O}_{8+\delta}$ using $60 \mathrm{~ns}$ time-scale short-pulse interlayer tunneling spectroscopy," Physical Review B, vol. 73, no. 18, Article ID 184512, 2006.

[461] Y. Yamada and M. Suzuki, "Interlayer tunneling spectroscopy for deeply underdoped $\mathrm{Bi}_{2} \mathrm{Sr}_{2} \mathrm{CaCu}_{2} \mathrm{O}_{8+\delta}$ : spectroscopic evidence for inhomogeneous superconductivity," Physical Review B, vol. 74, Article ID 054508, 2006.

[462] S. Johnston, F. Vernay, and T. P. Devereaux, "Impact of an oxygen dopant in $\mathrm{Bi}_{2} \mathrm{Sr}_{2} \mathrm{CaCu}_{2} \mathrm{O}_{8+\delta}$," Europhysics Letters, vol. 86, p. 37007, 2009.

[463] J.-H. Ma, Z.-H. Pan, F. C. Niestemski, et al., "Coexistence of competing orders with two energy gaps in real and momentum space in the high temperature superconductor $\mathrm{Bi}_{2} \mathrm{Sr}_{2-x} \mathrm{La}_{x} \mathrm{CuO}_{6+\delta}$," Physical Review Letters, vol. 101, no. 20, Article ID 207002, 2008.

[464] J. L. Tallon and J. W. Loram, "Comment on "thermodynamic transitions in inhomogeneous $d$-wave superconductors"," Physical Review B, vol. 79, no. 9, Article ID 096501, 2009.

[465] A. Gutiérrez-Sosa, U. Bangert, and W. R. Flavell, "Investigations of chemical and electronic inhomogeneities in $\mathrm{BaPb}_{1-x} \mathrm{Bi}_{x} \mathrm{O}_{3}$ via highly spatially resolved electron energy loss spectroscopy," Journal of Applied Physics, vol. 94, no. 10, pp. 6639-6643, 2003.

[466] I. Roshchin, V. Stepankin, and A. Kuznetsov, "Reentrant superconducting transport behavior of single grain boundary Josephson junction in $\mathrm{BaPb}_{1-x} \mathrm{Bi}_{x} \mathrm{O}_{3}$ bicrystals," Journal of Low Temperature Physics, vol. 100, no. 3-4, pp. 229-240, 1995.
[467] E. L. Nagaev, "Ground state and anomalous magnetic moment of conduction electrons in an antiferromagnetic semiconductor," Pis'ma Zhurnal Eksperimental'noi $i$ Teoreticheskoi Fiziki, vol. 6, p. 484, 1967.

[468] E. L. Nagaev, "Ferromagnetic domains in a semiconducting antiferromagnet," Zhurnal Éksperimental'noi i Teoreticheskoi Fiziki, vol. 54, p. 228, 1968.

[469] E. L. Nagaev, Physics of Magnetic Semiconductors, Nauka, Moscow, Russia, 1979.

[470] E. L. Nagaev, "Phase separation in high-temperature superconductors and related magnetic systems," Uspekhi Fizicheskikh Nauk, vol. 165, p. 529, 1995.

[471] M. A. Krivoglaz, "Electron states near the phase transition point and in disordered systems," Fizika Tverdogo Tela, vol. 11, p. 2230, 1969.

[472] M. A. Krivoglaz, "Fluctuonic states of electrons," Uspekhi Fizicheskikh Nauk, vol. 111, p. 617, 1973.

[473] M. A. Krivoglaz and A. I. Karasevskii, "Condensation in in the system of polarons or fluctuons with the formation of the nonhomogeneous state and peculiarities of conductance," Pis'ma Zhurnal Eksperimental'noi i Teoreticheskoi Fiziki, vol. 19, p. 454, 1974.

[474] M. A. Krivoglaz, Diffuse Scattering of X-Rays and Neutrons by Fluctuations, Springer, Berlin, Germany, 1996.

[475] J. M. Tranquada, B. J. Sternlieb, J. D. Axe, Y. Nakamura, and S. Uchida, "Evidence for stripe correlations of spins and holes in copper oxide superconductors," Nature, vol. 375, no. 6532, pp. 561-563, 1995.

[476] J. M. Tranquada, "Phase separation, charge segregation and superconductivity in layered cuprates," in Neutron Scattering in Layered Copper-Oxide Superconductors, A. Furrer, Ed., pp. 225-260, Kluwer Academic Publishers, Dordrecht, The Netherlands, 1998.

[477] M. B. Salamon and M. Jaime, "The physics of manganites: structure and transport," Reviews of Modern Physics, vol. 73, no. 3, pp. 583-628, 2001.

[478] A. Damascelli, Z. Hussain, and Z.-X. Shen, "Angle-resolved photoemission studies of the cuprate superconductors," Reviews of Modern Physics, vol. 75, no. 2, pp. 473-541, 2003.

[479] T. Yanagisawa, "Lattice distortions and stripes in the underdoped region of high- $T_{c}$ cuprates," Journal of Physics A, vol. 36, no. 35, pp. 9337-9349, 2003.

[480] J. M. Tranquada, H. Woo, T. G. Perring, et al., "Quantum magnetic excitations from stripes in copper oxide superconductors," Nature, vol. 429, no. 6991, pp. 534-538, 2004.

[481] O. N. Bakharev, I. M. Abu-Shiekah, H. B. Brom, A. A. Nugroho, I. P. McCulloch, and J. Zaanen, "NMR evidence for a two-step phase separation in $\mathrm{Nd}_{1.85} \mathrm{Ce}_{0.15} \mathrm{CuO}_{4-\delta}$," Physical Review Letters, vol. 93, no. 3, Article ID 037002, 1 pages, 2004.

[482] C. J. Zhang and H. Oyanagi, "Effects of local lattice disorder on the superconductivity studied by XAFS," Journal of Physics: Conference Series, vol. 108, no. 1, Article ID 012048, 2008.

[483] H. Oyanagi, C. Zhang, A. Tsukada, and M. Naito, "Lattice instability in high temperature superconducting cuprates probed by X-ray absorption spectroscopy," Journal of Physics: Conference Series, vol. 108, no. 1, Article ID 012038, 2008.

[484] Y. Koike, T. Adachi, Y. Tanabe, K. Omori, T. Noji, and H. Sato, "Inhomogeneous superconductivity in both underdoped and overdoped regimes of high- $T_{c}$ cuprates," Journal of Physics: Conference Series, vol. 108, no. 1, Article ID 012003, 2008.

[485] H. Keller, A. Bussmann-Holder, and K. A. Müller, "JahnTeller physics and high- $T_{c}$ superconductivity," Materials Today, vol. 11, no. 9, pp. 38-46, 2008. 
[486] J. C. Phillips, A. Saxena, and A. R. Bishop, "Pseudogaps, dopants, and strong disorder in cuprate high-temperature superconductors," Reports on Progress in Physics, vol. 66, no. 12, pp. 2111-2182, 2003.

[487] R. A. Klemm, "The pseudogap and the superconducting order parameter in inhomogeneous $\mathrm{Bi}_{2} \mathrm{Sr}_{2} \mathrm{CaCu}_{2} \mathrm{O}_{8+\delta}$, Journal of Superconductivity and Novel Magnetism, vol. 17, no. 1, pp. 69-74, 2004.

[488] J. C. Phillips, "Quantum percolation in cuprate hightemperature superconductors," Proceedings of the National Academy of Sciences of the United States of America, vol. 105, no. 29, pp. 9917-9919, 2008.

[489] F. V. Kusmartsev and M. Saarela, "What is the most important for a nanoscale structure formations in HTSC? Spin, phonon or third way in Coulomb interaction and correlations?" Journal of Physics: Conference Series, vol. 108, no. 1, Article ID 012029, 2008.

[490] F. V. Kusmartsev and M. Saarela, "Two-component physics of cuprates and superconductor-insulator transitions," Superconductor Science and Technology, vol. 22, no. 1, Article ID 014008, 2009.

[491] B. Fine and T. Egami, "Intermediate spin-charge order in the cuprates," Journal of Physics: Conference Series, vol. 108, no. 1, Article ID 012005, 2008.

[492] M. Müller and B. I. Shklovskii, "Compensation-driven superconductor-insulator transition," Physical Review B, vol. 79, no. 13, Article ID 134504, 2009.

[493] A. S. Alexandrov, "Phase separation of electrons strongly coupled with phonons in cuprates and manganites," Journal of Superconductivity and Novel Magnetism, vol. 22, no. 2, pp. 95-101, 2009.

[494] J. T. Park, D. S. Inosov, Ch. Niedermayer, et al., "Electronic phase separation in the slightly underdoped iron pnictide superconductor $\mathrm{Ba}_{1-x} \mathrm{~K}_{x} \mathrm{Fe}_{2} \mathrm{As}_{2}$," Physical Review Letters, vol. 102, no. 11, Article ID 117006, 2009.

[495] B. Sacépé, C. Chapelier, T. I. Baturina, V. M. Vinokur, M. R. Baklanov, and M. Sanquer, "Disorder-induced inhomogeneities of the superconducting state close to the superconductor-insulator transition," Physical Review Letters, vol. 101, no. 15, Article ID 157006, 2008.

[496] H. L. Edwards, A. L. Barr, J. T. Markert, and A. L. De Lozanne, "Modulations in the $\mathrm{CuO}$ chain layer of $\mathrm{YBa}_{2} \mathrm{Cu}_{3} \mathrm{O}_{7-\delta}$ : charge density waves?" Physical Review Letters, vol. 73, no. 8, pp. 1154-1157, 1994.

[497] D. J. Derro, E. W. Hudson, K. M. Lang, et al., "Nanoscale one-dimensional scattering resonances in the $\mathrm{CuO}$ chains of $\mathrm{YBa}_{2} \mathrm{Cu}_{3} \mathrm{O}_{6+x}$," Physical Review Letters, vol. 88, no. 9, Article ID 097002, 4 pages, 2002.

[498] A. A. Abrikosov, Fundamentals of the Theory of Metals, North-Holland, Amsterdam, The Netherlands, 1987.

[499] K. McElroy, R. W. Simmonds, J. E. Hoffman, et al., "Relating atomic-scale electronic phenomena to wave-like quasiparticle states in superconducting $\mathrm{Bi}_{2} \mathrm{Sr}_{2} \mathrm{CaCu}_{2} \mathrm{O}_{8+\delta}$," Nature, vol. 422, no. 6932, pp. 592-596, 2003.

[500] T. Pereg-Barnea and M. Franz, "Theory of quasiparticle interference patterns in the pseudogap phase of the cuprate superconductors," Physical Review B, vol. 68, no. 18, Article ID 180506, 4 pages, 2003.

[501] T. Pereg-Barnea and M. Franz, "Quasiparticle interference patterns as a test for the nature of the pseudogap phase in the cuprate superconductors," International Journal of Modern Physics B, vol. 19, no. 4, pp. 731-761, 2005.
[502] T. S. Nunner, W. Chen, B. M. Andersen, A. Melikyan, and P. J. Hirschfeld, "Fourier transform spectroscopy of $d$ wave quasiparticles in the presence of atomic scale pairing disorder," Physical Review B, vol. 73, no. 10, Article ID 104511, 7 pages, 2006.

[503] T. Hanaguri, Y. Kohsaka, J. C. Davis, et al., "Quasiparticle interference and superconducting gap in $\mathrm{Ca}_{2-x} \mathrm{Na}_{x} \mathrm{CuO}_{2} \mathrm{Cl}_{2}$," Nature Physics, vol. 3, no. 12, pp. 865-871, 2007.

[504] Y. Kohsaka, C. Taylor, P. Wahl, et al., "How Cooper pairs vanish approaching the Mott insulator in $\mathrm{Bi}_{2} \mathrm{Sr}_{2} \mathrm{CaCu}_{2} \mathrm{O}_{8+\delta}$," Nature, vol. 454, no. 7208, pp. 1072-1078, 2008.

[505] T. Hanaguri, Y. Kohsaka, M. Ono, et al., "Coherence factors in a high- $T_{c}$ cuprate probed by quasi-particle scattering off vortices," Science, vol. 323, no. 5916, pp. 923-926, 2009.

[506] C. Howald, H. Eisaki, N. Kaneko, M. Greven, and A. Kapitulnik, "Periodic density-of-states modulations in superconducting $\mathrm{Bi}_{2} \mathrm{Sr}_{2} \mathrm{CaCu}_{2} \mathrm{O}_{8+\delta}$," Physical Review B, vol. 67, no. 1, Article ID 014533, 10 pages, 2003.

[507] T. Hanaguri, C. Lupien, Y. Kohsaka, et al., "A 'checkerboard' electronic crystal state in lightly holedoped $\mathrm{Ca}_{2-x} \mathrm{Na}_{x} \mathrm{CuO}_{2} \mathrm{Cl}_{2}$," Nature, vol. 430, no. 7003, pp. 1001-1005, 2004.

[508] Y. Kohsaka, C. Taylor, K. Fujita, et al., "An intrinsic bondcentered electronic glass with unidirectional domains in underdoped cuprates," Science, vol. 315, no. 5817, pp. 13801385, 2007.

[509] M. F. Crommie, C. P. Lutz, and D. M. Eigler, "Imaging standing waves in a two-dimensional electron gas," Nature, vol. 363, no. 6429, pp. 524-527, 1993.

[510] G. A. Fiete and E. J. Heller, "Colloquium: theory of quantum corrals and quantum mirages," Reviews of Modern Physics, vol. 75, no. 3, pp. 933-948, 2003.

[511] J. Friedel, "Metallic alloys," Il Nuovo Cimento, vol. 7, supplement 2, pp. 287-311, 1958.

[512] J. E. Hoffman, K. McElroy, D.-H. Lee, et al., "Imaging quasiparticle interference in $\mathrm{Bi}_{2} \mathrm{Sr}_{2} \mathrm{CaCu}_{2} \mathrm{O}_{8+\delta}$," Science, vol. 297, no. 5584, pp. 1148-1151, 2002.

[513] A. M. Gabovich and E. A. Pashitskii, "Polarization operator of the superconducting electron gas. Kohn anomalies and charge screening in superconductors," Ukrainskii Fizychnyi Zhurnal, vol. 18, p. 549, 1973.

[514] J. P. Hurault, "Note sur les polarisations de spin et de charge autour d'une impurete dans un supraconducteur," Journal de Physique, vol. 26, p. 252, 1965.

[515] A. M. Gabovich, L. G. Il'chenko, and E. A. Pashitskii, Fizika Tverdogo Tela, vol. 21, p. 2191, 1979.

[516] G. Grüner, Density Waves in Solids, Addison-Wesley, Reading, Mass, USA, 1994.

[517] T. M. Rice and G. K. Scott, "New mechanism for a chargedensity-wave instability," Physical Review Letters, vol. 35, no. 2, pp. 120-123, 1975.

[518] R. S. Markiewicz, "A survey of the Van Hove scenario for high- $T_{c}$ superconductivity with special emphasis on pseudogaps and striped phases," Journal of Physics and Chemistry of Solids, vol. 58, no. 8, pp. 1179-1310, 1997.

[519] R. Peierls, "Zur Theorie der elektrischen urnd therrnlschen Leitfähigkeit von Metallen," Annals of Physics, vol. 4, p. 121, 1930.

[520] R. E. Peierls, Quantum Theory of Solids, Clarendon Press, Oxford, UK, 1955.

[521] L. V. Keldysh and Yu. V. Kopaev, Fizika Tverdogo Tela, vol. 6, p. 2791, 1964. 
[522] B. I. Halperin and T. M. Rice, "The excitonic state at the semiconductor-semimetal transition," in Solid State Physics, vol. 21, p. 115, Academic Press, New York, NY, USA, 1968.

[523] A. N. Kozlov and L. A. Maksimov, "On the metal-insulator phase transition. Divalent crystal," Zhurnal Eksperimental'noi i Teoreticheskoi Fiziki, vol. 48, p. 1184, 1965.

[524] T. Ishiguro and K. Yamaji, Organic Superconductors, Springer, Berlin, Germany, 1990.

[525] C. Schlenker, J. Dumas, M. Greenblatt, and S. van Smaalen, Eds., Physics and Chemistry of Low-Dimensional Inorganic Conductors, Plenum Press, New York, NY, USA, 1996.

[526] B. Kyung, "Mean-field study of the interplay between antiferromagnetism and $d$-wave superconductivity," Physical Review B, vol. 62, no. 13, pp. 9083-9088, 2000.

[527] H. Wilhelm, D. Jaccard, R. Duprat, et al., "The case for universality of the phase diagram of the Fabre and Bechgaard salts," European Physical Journal B, vol. 21, no. 2, pp. 175-183, 2001.

[528] C. Colin, C. R. Pasquier, and K. Bechgaard, "Multi-phase coexistence in the organic conductor (TMTSF) ${ }_{2} \mathrm{ReO}_{4}$," Journal de Physique, vol. 131, pp. 313-314, 2005.

[529] A. Aperis, G. Varelogiannis, P. B. Littlewood, and B. D. Simons, "Coexistence of spin density wave, $d$-wave singlet and staggered $\pi$-triplet superconductivity," Journal of Physics: Condensed Matter, vol. 20, Article ID 434235, 5 pages, 2008.

[530] J. T. Devreese, R. P. Evrard, and V. E. van Doren, Eds., Highly Conducting One-Dimensional Solids, Plenum Press, New York, NY, USA, 1979.

[531] P. Monceau, Ed., Electronic Properties of Inorganic Quasi-OneDimensional Compounds_Part II: Experimental, D. Reidel, Dordrecht, The Netherlands, 1985.

[532] S. Roth and D. Carroll, One-Dimensional Metals, Wiley-VCH, Weinheim, Germany, 2004.

[533] M. J. Rice and Yu. N. Gartstein, "The excitonic ground state of the half-filled Peierls insulator," Journal of Physics: Condensed Matter, vol. 17, no. 29, pp. 4615-4620, 2005.

[534] W. Kohn and D. Sherrington, "Two kinds of bosons and bose condensates," Reviews of Modern Physics, vol. 42, no. 1, pp. $1-11,1970$.

[535] H. Cercellier, C. Monney, F. Clerc, et al., "Evidence for an excitonic insulator phase in 1 -TiSe ${ }_{2}$," Physical Review Letters, vol. 99, no. 14, Article ID 146403, 4 pages, 2007.

[536] C. Monney, H. Cercellier, F. Clerc, et al., "Spontaneous exciton condensation in $1 T$-TiSe 2 : BCS-like approach," Physical Review B, vol. 79, no. 4, Article ID 045116, 11 pages, 2009.

[537] B. Bucher, P. Steiner, and P. Wachter, "Excitonic insulator phase in $\mathrm{TmSe}_{0.45} \mathrm{Te}_{0.55}$," Physical Review Letters, vol. 67, no. 19, pp. 2717-2720, 1991.

[538] P. Wachter, A. Jung, and P. Steiner, "Pressure-driven metalinsulator transition in La-doped SmS: excitonic condensation," Physical Review B, vol. 51, no. 8, pp. 5542-5545, 1995.

[539] Y. Wakisaka, T. Sudayama, K. Takubo, et al., "Excitonic insulator state in $\mathrm{Ta}_{2} \mathrm{NiSe}_{5}$ probed by photoemission spectroscopy," Physical Review Letters, vol. 103, no. 2, Article ID 026402, 4 pages, 2009.

[540] J. Zaanen, "Superconductivity: quantum stripe search," Nature, vol. 440, no. 7088, pp. 1118-1119, 2006.

[541] P. Wróbel, "Checkerboard or stripes: hard-core bosons on the checkerboard lattice as a model of charge ordering in planar cuprates," Physical Review B, vol. 74, no. 1, Article ID 014507, 8 pages, 2006.
[542] V. Cvetkovic, Z. Nussinov, S. Mukhin, and J. Zaanen, "Observing the fluctuating stripes in high- $T_{c}$ superconductors," Europhysics Letters, vol. 81, no. 2, Article ID 27001, 6 pages, 2008.

[543] C. W. Misner, K. S. Thorne, and W. H. Zurek, "John Wheeler, relativity, and quantum information," Physics Today, vol. 62, no. 4, pp. 40-46, 2009.

[544] P. Abbamonte, A. Rusydi, S. Smadici, G. D. Gu, G. A. Sawatzky, and D. L. Feng, "Spatially modulated 'Mottness' in $\mathrm{La}_{2-x} \mathrm{Ba}_{x} \mathrm{CuO}_{4}$," Nature Physics, vol. 1, pp. 155-158, 2005.

[545] M. Fujita, H. Goka, K. Yamada, and M. Matsuda, "Competition between charge- and spin-density-wave order and superconductivity in $\mathrm{La}_{1.875} \mathrm{Ba}_{0.125-x} \mathrm{Sr}_{x} \mathrm{CuO}_{4}$," Physical Review Letters, vol. 88, no. 16, Article ID 167008, 4 pages, 2002.

[546] J. Fink, E. Schierle, E. Weschke, et al., "Charge ordering in $\mathrm{La}_{1.8-x} \mathrm{Eu}_{0.2} \mathrm{Sr}_{x} \mathrm{CuO}_{4}$ studied by resonant soft X-ray diffraction," Physical Review B, vol. 79, no. 10, Article ID 100502, 4 pages, 2009.

[547] C. M. Varma, "Non-Fermi-liquid states and pairing instability of a general model of copper oxide metals," Physical Review B, vol. 55, no. 21, pp. 14554-14580, 1997.

[548] H. J. Schulz, "Fermi-surface instabilities of a generalized twodimensional Hubbard model," Physical Review B, vol. 39, no. 4, pp. 2940-2943, 1989.

[549] S. Chakravarty, R. B. Laughlin, D. K. Morr, and C. Nayak, "Hidden order in the cuprates," Physical Review B, vol. 63, no. 9, Article ID 094503, 10 pages, 2001.

[550] D. Jérome, C. Berthier, P. Molinié, and J. Rouxel, "Layer compounds. Charge density waves in transitions metal compounds. Electronic properties of transition metal dichalcogenides: connection between structural instabilities and superconductivity," Journal de Physique, vol. 37, pp. C4C125, 1976.

[551] O. Degtyareva, M. V. Magnitskaya, J. Kohanoff, et al., "Competition of charge-density waves and superconductivity in sulfur," Physical Review Letters, vol. 99, Article ID 155505, 4 pages, 2007.

[552] K. Kudo, N. Okumura, Y. Miyoshi, T. Nishizaki, T. Sasaki, and N. Kobayashi, "Narrow carrier concentration range of superconductivity and critical point of pseudogap formation temperature in $\mathrm{Pb}$-substituted $\mathrm{Bi}_{2} \mathrm{Sr}_{2} \mathrm{CuO}_{6+\delta}$, J Journal of the Physical Society of Japan, vol. 78, no. 8, Article ID 084722, 5 pages, 2009.

[553] H. Won and K. Maki, " $d$-wave superconductor as a model of high- $T_{c}$ superconductors," Physical Review B, vol. 49, no. 2, pp. 1397-1402, 1994.

[554] H. Won, K. Maki, and E. Puchkaryov, in High-T $T_{c}$ Superconductors and Related Materials: Material Science, Fundamental Properties, and Some Future Electronic Applications, S. L. Drechsler and T. Mishonov, Eds., p. 375, Kluwer Academic, Dordrecht, The Netherlands, 2001.

[555] H. Won, S. Haas, D. Parker, S. Telang, A. Ványolos, and K. Maki, in Lectures on the Physics of Highly Correlated Electron Systems IX: Ninth Training Course, A. Avella and F. Mancini, Eds., p. 3, American Institute of Physics, Melville, NY, USA, 2005.

[556] A. M. Gabovich and A. I. Voitenko, "Josephson tunnelling involving superconductors with charge-density waves," Journal of Physics: Condensed Matter, vol. 9, no. 19, pp. 39013920, 1997.

[557] A. M. Gabovich and A. I. Voitenko, "Asymmetrical tunneling between similar metallic junctions with charge-density or spin-density waves: the case of broken symmetry," Physical Review B, vol. 56, no. 13, pp. 7785-7788, 1997. 
[558] B. Mühlschlegel, "Die thermodynamischen Funktionen des Supraleiters," Zeitschrift für Physik A, vol. 155, no. 3, pp. 313327, 1959.

[559] V. M. Krasnov, "Temperature dependence of the bulk energy gap in underdoped $\mathrm{Bi}_{2} \mathrm{Sr}_{2} \mathrm{CaCu}_{2} \mathrm{O}_{8+\delta}$ : evidence for the meanfield superconducting transition," Physical Review B, vol. 79, no. 21, Article ID 214510, 17 pages, 2009.

[560] M. Suzuki and T. Watanabe, "Discriminating the superconducting gap from the pseudogap in $\mathrm{Bi}_{2} \mathrm{Sr}_{2} \mathrm{CaCu}_{2} \mathrm{O}_{8+\delta}$ by interlayer tunneling spectroscopy," Physical Review Letters, vol. 85, no. 22, pp. 4787-4790, 2000.

[561] T. Ekino, S. Hashimoto, T. Takasaki, and H. Fujii, "Tunneling spectroscopy of the normal-state gap in (Bi, $\mathrm{Pb})_{2} \mathrm{Sr}_{2} \mathrm{Ca}_{2} \mathrm{Cu}_{3} \mathrm{O}_{10+\delta}$," Physical Review B, vol. 64, no. 9, Article ID 092510, 4 pages, 2001.

[562] T. Ekino, Y. Sezaki, S. Hashimoto, and H. Fujii, "Observation of the energy gap above $(\mathrm{Bi}, \mathrm{Pb})_{2} \mathrm{Sr}_{2} \mathrm{Ca}_{2} \mathrm{Cu}_{3} \mathrm{O}_{10+\delta}$ using break-junction tunneling," Journal of Low Temperature Physics, vol. 117, no. 3-4, pp. 359-363, 1999.

[563] A. Sugimoto, T. Ekino, and H. Eisaki, "Nanoscale modulation of local barrier height on Bi-based cuprate superconductors observed by scanning tunneling microscopy/spectroscopy," Journal of the Physical Society of Japan, vol. 77, no. 4, Article ID 043705, 4 pages, 2008.

[564] J. W. Alldredge, J. Lee, K. McElroy, et al., "Evolution of the electronic excitation spectrum with strongly diminishing hole density in superconducting $\mathrm{Bi}_{2} \mathrm{Sr}_{2} \mathrm{CaCu}_{2} \mathrm{O}_{8+\delta}$," Nature Physics, vol. 4, no. 4, pp. 319-326, 2008.

[565] Y. Kohsaka, K. Iwaya, S. Satow, M. Azuma, M. Takano, and H. Takagi, "Imaging nanoscale electronic inhomogeneity in the lightly doped mott insulator $\mathrm{Ca}_{2-x} \mathrm{Na}_{x} \mathrm{CuO}_{2} \mathrm{Cl}_{2}$," Physical Review Letters, vol. 93, no. 9, Article ID 097004, 4 pages, 2004.

[566] A. M. Gabovich and A. S. Shpigel, Fizika Tverdogo Tela, vol. 26, p. 1569, 1984.

[567] R. Escudero, F. Morales, and S. Bernès, "Specific heat studies of pure $\mathrm{Nb}_{3} \mathrm{Sn}$ single crystals at low temperature," Journal of Physics: Condensed Matter, vol. 21, no. 32, Article ID 325701, 7 pages, 2009.

[568] N. E. Phillips, R. A. Fisher, and J. E. Gordon, in Progress in Low Temperature Physics, vol. 13, p. 267, Elsevier, Amsterdam, The Netherlands, 1992.

[569] J. W. Loram, K. A. Mirza, W. Y. Liang, and J. Osborne, "A systematic study of the specific heat anomaly in $\mathrm{La}_{2-x} \mathrm{Sr}_{x} \mathrm{CuO}_{4}$," Physica C, vol. 162-164, part 1, pp. 498-499, 1989.

[570] J. L. Tallon and J. W. Loram, "Doping dependence of $T^{*}-$ what is the real high- $T_{c}$ phase diagram?" Physica $C$, vol. 349, no. 1-2, pp. 53-68, 2001.

[571] H.-H. Wen, G. Mu, H. Luo, et al., "Specific-heat measurement of a residual superconducting state in the normal state of underdoped $\mathrm{Bi}_{2} \mathrm{Sr}_{2-x} \mathrm{La}_{x} \mathrm{CuO}_{6+\delta}$ cuprate superconductors," Physical Review Letters, vol. 103, no. 6, Article ID 067002, 4 pages, 2009.

[572] B. Krunavakarn, S. Kaskamalas, and S. Yoksan, "Thermodynamic properties of a BCS superconductor," Physica C, vol. 338, no. 4, pp. 305-315, 2000.

[573] H. Balci and R. L. Greene, "Thermodynamic properties of $\operatorname{Pr}_{1.85} \mathrm{Ce}_{0.15} \mathrm{CuO}_{4-\delta}$," Physical Review B, vol. 70, no. 14, Article ID 140508, 4 pages, 2004.

[574] C. E. Methfessel, A. R. Stewart, B. T. Matthias, and C. K. N. Patel, "Why is there no bulk specific heat anomaly at the superconducting transition temperature of $\mathrm{BaPb}_{1-x} \mathrm{Bi}_{x} \mathrm{O}_{3}$ ?" Proceedings of the National Academy of Sciences of the United States of America, vol. 77, no. 11, pp. 6307-6308, 1980.
[575] S. Tanaka, K. Kitazawa, and T. Tani, Annual Report of the Engineering Research Institute, Faculty of Engineering, University of Tokyo, vol. 41, p. 131, 1982.

[576] M. Sato, H. Fujishita, and S. Hoshino, "Specific heat anomaly of $\mathrm{BaPb}_{1-x} \mathrm{Bi}_{x} \mathrm{O}_{3}$ at the superconducting transition," Journal of Physics C, vol. 16, no. 13, pp. L417-L421, 1983.

[577] B. B. Schwartz and S. Foner, "Large-scale applications of superconductivity," Physics Today, vol. 30, p. 34, 1977.

[578] D. Larbalestier, G. Fisk, B. Montgomery, and D. Hawksworth, "High-field superconductivity," Physics Today, vol. 39, no. 3, pp. 24-35, 1986.

[579] D. Larbalestier, A. Gurevich, D. M. Feldmann, and A. Polyanskii, "High- $T_{c}$ superconducting materials for electric power applications," Nature, vol. 414, no. 6861, pp. 368-377, 2001.

[580] S. Kang, A. Goyal, J. Li, et al., "High-performance high- $T_{c}$ superconducting wires," Science, vol. 311, no. 5769, pp. 19111914, 2006.

[581] A. M. Clogston, "Upper limit for the critical field in hard superconductors," Physical Review Letters, vol. 9, no. 6, pp. 266-267, 1962.

[582] B. S. Chandrasekhar, "A note on the maximum critical field of high-field superconductors," Applied Physics Letters, vol. 1, no. 1, pp. 7-8, 1962.

[583] P. Fulde, "High field superconductivity in thin films," Advances in Physics, vol. 22, no. 6, pp. 667-719, 1973.

[584] R. C. Bruno and B. B. Schwartz, "Magnetic field splitting of the density of states of thin superconductors," Physical Review $B$, vol. 8, no. 7, pp. 3161-3178, 1973.

[585] P. M. Tedrow and R. Meservey, "Experimental test of the theory of high-field superconductivity," Physical Review Letters, vol. 43, no. 5, pp. 384-387, 1979.

[586] R. G. Dias and J. A. Silva, "Huge metastability in high- $T_{c}$ superconductors induced by parallel magnetic field," Physical Review B, vol. 67, no. 9, Article ID 092511, 4 pages, 2003.

[587] A. M. Gabovich, E. A. Pashitskii, and A. S. Shpigel, "Exceeding of paramagnetic limit in superconductors with dielectric gap on the Fermi surface," Zhurnal Eksperimental'noi $i$ Teoreticheskoi Fiziki, vol. 28, p. 302, 1978.

[588] A. M. Gabovich, E. A. Pashitskii, and A. S. Shpigel, "Paramagnetic limit of superconductors with a dielectric gap on the Fermi surface," Zhurnal Eksperimental'noi $i$ Teoreticheskoi Fiziki, vol. 77, p. 1157, 1979.

[589] A. Gabovich, A. I. Voitenko, and T. Ekino, "Enhanced paramagnetic limit of the upper critical magnetic field for superconductors with charge-density waves," Journal of Physics: Condensed Matter, vol. 16, no. 21, pp. 3681-3690, 2004.

[590] R. H. McKenzie, "Is the ground state of alpha-(BEDT$\mathrm{TTF})_{2} \mathrm{MHg}(\mathrm{SCN})_{4}[\mathrm{M}=\mathrm{K}, \mathrm{Rb}, \mathrm{Tl}]$ a charge-density wave or a spin-density wave?" http://arxiv.org/abs/cond-mat/9706235.

[591] N. Harrison, "Destabilization of a charge-density wave by an oscillatory chemical potential," Physical Review Letters, vol. 83, no. 7, pp. 1395-1398, 1999.

[592] J. S. Qualls, L. Balicas, J. S. Brooks, N. Harrison, L. K. Montgomery, and M. Tokumoto, "Competition between Pauli and orbital effects in a charge-density-wave system," Physical Review B, vol. 62, no. 15, pp. 10008-10012, 2000.

[593] L. D. Landau and E. M. Lifshits, Electrodynamics of Continuous Media, Pergamon, New York, NY, USA, 1984.

[594] N. P. Ong and P. Monceau, "Anomalous transport properties of a linear-chain metal: $\mathrm{NbSe}_{3}$," Physical Review B, vol. 16, no. 8, pp. 3443-3455, 1977. 
[595] S. Sugai, Y. Takayanagi, and N. Hayamizu, "Phason and amplitudon in the charge-density-wave phase of onedimensional charge stripes in $\mathrm{La}_{2-x} \mathrm{Sr}_{x} \mathrm{CuO}_{4}$, Physical Review Letters, vol. 96, no. 13, Article ID 137003, 4 pages, 2006.

[596] A. M. Gabovich and A. I. Voitenko, "Nonstationary Josephson effect for superconductors with charge-density waves," Physical Review B, vol. 55, no. 2, pp. 1081-1099, 1997.

[597] N. Miyakawa, J. F. Zasadzinski, L. Ozyuzer, et al., "Predominantly superconducting origin of large energy gaps in underdoped $\mathrm{Bi}_{2} \mathrm{Sr}_{2} \mathrm{CaCu}_{2} \mathrm{O}_{8+\delta}$ from tunneling spectroscopy," Physical Review Letters, vol. 83, no. 5, pp. 1018-1021, 1999.

[598] Ch. Renner, B. Revaz, J.-Y. Genoud, K. Kadowakj, and $\varnothing$. Fischer, "Pseudogap precursor of the superconducting gap in under- and overdoped $\mathrm{Bi}_{2} \mathrm{Sr}_{2} \mathrm{CaCu}_{2} \mathrm{O}_{8+\delta}$," Physical Review Letters, vol. 80, no. 1, pp. 149-152, 1998.

[599] A. K. Gupta and K.-W. Ng, "ab-plane tunneling spectroscopy of underdoped $\mathrm{Bi}_{2} \mathrm{Sr}_{2} \mathrm{CaCu}_{2} \mathrm{O}_{y}$," Physical Review B, vol. 58, no. 14, pp. R8901-R8904, 1998.

[600] J. F. Zasadzinski, L. Ozyuzer, N. Miyakawa, K. E. Gray, D. G. Hinks, and C. Kendziora, "Correlation of tunneling spectra in $\mathrm{Bi}_{2} \mathrm{Sr}_{2} \mathrm{CaCu}_{2} \mathrm{O}_{8+\delta}$ with the resonance spin excitation," Physical Review Letters, vol. 87, no. 6, Article ID 067005, 4 pages, 2001.

[601] D. N. Dias, E. S. Caixeiro, and E. V. L. de Mello, "Magnetic properties of cuprate superconductors based on a phase separation theory," Physica C, vol. 468, no. 6, pp. 480-486, 2008.

[602] A. Bille, R. A. Klemm, and K. Scharnberg, "Models of c-axis twist Josephson tunneling," Physical Review B, vol. 64, no. 17, Article ID 174507, 23 pages, 2001.

[603] A. I. Larkin and Yu. N. Ovchinnikov, "Tunnel effect between superconductors in an alternating field," Zhurnal Eksperimental'noi i Teoreticheskoi Fiziki, vol. 51, p. 1535, 1966.

[604] W. A. Roshen, "Thermodynamic properties of chargedensity waves," Physical Review B, vol. 31, no. 11, pp. 72967305, 1985.

[605] I. Bâldea, "Reentrant charge-density wave in the onedimensional system with impurities-a self-consistent approach," Physica Scripta, vol. 42, no. 6, pp. 749-760, 1990.

[606] J. Zittartz, "Theory of the excitonic insulator in the presence of normal impurities," Physical Review, vol. 164, no. 2, pp. 575-582, 1967.

[607] A. A. Abrikosov and L. P. Gor'kov, "On the theory of superconducting alloys. 1. Electrodynamics of alloys at the absolute zero," Zhurnal Eksperimental'noi i Teoreticheskoi Fiziki, vol. 35, p. 1558, 1958.

[608] A. A. Abrikosov and L. P. Gor'kov, "Superconducting alloys at temperatures above the absolute zero," Zhurnal Eksperimental'noi i Teoreticheskoi Fiziki, vol. 36, p. 319, 1959.

[609] P. W. Anderson, "Theory of dirty superconductors," Journal of Physics and Chemistry of Solids, vol. 11, no. 1-2, pp. 26-30, 1959.

[610] H. Kim, G. Preosti, and P. Muzikar, "Penetration depth and impurity scattering in unconventional superconductors: $T=$ 0 results," Physical Review B, vol. 49, no. 5, pp. 3544-3547, 1994.

[611] Y. Sun and K. Maki, "Impurity effects in $d$-wave superconductors," Physical Review B, vol. 51, no. 9, pp. 6059-6063, 1995.

[612] D. Manske, I. Eremin, and K. H. Bennemann, "Analysis of the elementary excitations in high- $T_{c}$ cuprates: explanation of the new energy scale observed by angle-resolved photoemission spectroscopy," Physical Review Letters, vol. 87, no. 17, Article ID 177005, 4 pages, 2001.
[613] D. S. Inosov, S. V. Borisenko, I. Eremin, et al., "Relation between the one-particle spectral function and dynamic spin susceptibility of superconducting $\mathrm{Bi}_{2} \mathrm{Sr}_{2} \mathrm{CaCu}_{2} \mathrm{O}_{8-\delta}$," Physical Review B, vol. 75, no. 17, Article ID 172505, 4 pages, 2007.

[614] A. N. Pasupathy, A. Pushp, K. K. Gomes, et al., "Electronic origin of the inhomogeneous pairing interaction in the high$T_{c}$ superconductor $\mathrm{Bi}_{2} \mathrm{Sr}_{2} \mathrm{CaCu}_{2} \mathrm{O}_{8+\delta}$," Science, vol. 320, no. 5873, pp. 196-201, 2008.

[615] M.-H. Bae, J.-H. Park, J.-H. Choi, H.-J. Lee, and K.-S. Park, "Pseudogap behavior revealed in interlayer tunneling in overdoped $\mathrm{Bi}_{2} \mathrm{Sr}_{2} \mathrm{CaCu}_{2} \mathrm{O}_{8+x}$," Physical Review B, vol. 77, no. 9, Article ID 094519, 8 pages, 2008.

[616] A. Kussmaul, E. S. Hellman, E. H. Hartford Jr., and P. M. Tedrow, "Superconductor-insulator-superconductor tunneling in $\mathrm{Ba}_{1-x} \mathrm{~K}_{x} \mathrm{BiO}_{3}$ grain boundaries," Applied Physics Letters, vol. 63, no. 20, pp. 2824-2826, 1993.

[617] A. Mourachkine, "Origin of dips in tunneling dI/dV characteristics of cuprates," Physica C, vol. 460-462, pp. 956-957, 2007.

[618] D. J. van Harlingen, "Phase-sensitive tests of the symmetry of the pairing state in the high-temperature superconductorsevidence for $d_{x^{2}-y^{2}}$ symmetry," Reviews of Modern Physics, vol. 67, no. 2, pp. 515-535, 1995.

[619] J. Mannhart and P. Chaudhari, "High- $T_{c}$ bicrystal grain boundaries," Physics Today, vol. 54, no. 11, pp. 48-53, 2001.

[620] N. E. Hussey, M. Abdel-Jawad, A. Carrington, A. P. Mackenzie, and L. Balicas, "A coherent three-dimensional Fermi surface in a high-transition-temperature superconductor," Nature, vol. 425, no. 6960, pp. 814-817, 2003.

[621] K. Yang and S. L. Sondhi, "Response of a $d_{x^{2}-y^{2}}$ superconductor to a Zeeman magnetic field," Physical Review B, vol. 57, no. 14, pp. 8566-8570, 1998.

[622] A. Kanigel, M. R. Norman, M. Randeria, et al., "Evolution of the pseudogap from Fermi arcs to the nodal liquid," Nature Physics, vol. 2, no. 7, pp. 447-451, 2006.

[623] M. Hashimoto, T. Yoshida, K. Tanaka, et al., "Distinct doping dependences of the pseudogap and superconducting gap of $\mathrm{La}_{2-x} \mathrm{Sr}_{x} \mathrm{CuO}_{4}$ cuprate superconductors," Physical Review B, vol. 75, no. 14, Article ID 140503, 4 pages, 2007.

[624] K. McElroy, "Death of a Fermi surface," Nature Physics, vol. 2, no. 7, pp. 441-442, 2006.

[625] Y. H. Liu, K. Takeyama, T. Kurosawa, N. Momono, M. Oda, and M. Ido, " $4 a \times 4 a$ electronic charge order enhanced in the inhomogeneous pseudogap state of $\mathrm{Bi}_{2} \mathrm{Sr}_{2} \mathrm{CaCu}_{2} \mathrm{O}_{8+\delta}$," Physical Review B, vol. 75, no. 21, Article ID 212507, 4 pages, 2007.

[626] A. Pushp, C. V. Parker, A. N. Pasupathy, et al., "Extending universal nodal excitations optimizes superconductivity in $\mathrm{Bi}_{2} \mathrm{Sr}_{2} \mathrm{CaCu}_{2} \mathrm{O}_{8+\delta}$, Science, vol. 324, no. 5935, pp. 16891693, 2009.

[627] D. Einzel, "Universal parameters in the response of unconventional superconductors," Journal of Low Temperature Physics, vol. 126, no. 3-4, pp. 867-880, 2002.

[628] D. Einzel, "Analytic two-fluid description of unconventional superconductivity," Journal of Low Temperature Physics, vol. 131, no. 1-2, pp. 1-24, 2003.

[629] B.-L. Yu, J. C. F. Wang, A. D. Beyer, et al., "Possible competing order-induced Fermi arcs in cuprate superconductors," Solid State Communications, vol. 149, no. 7-8, pp. 261-265, 2009.

[630] A. Furrer, "Admixture of an $s$-wave component to the $d$ wave gap symmetry in high-temperature superconductors," Journal of Superconductivity and Novel Magnetism, vol. 21, no. 1, pp. 1-5, 2008. 
[631] T. Hasegawa, H. Ikuta, and K. Kitazawa, "Tunneling spectroscopy of oxide superconductors," in Physical Properties of High Temperature Superconductors III, D. M. Ginsberg, Ed., p. 525, World Scientific, Singapore, 1992.

[632] M. Oda, N. Momono, and M. Ido, "What is the energy scale in determining the $T_{c}$ of cuprate superconductivity?" Superconductor Science and Technology, vol. 13, no. 11, pp. R139-R146, 2000.

[633] B. T. Geilikman, V. Z. Kresin, and N. F. Masharov, "Transition temperature and energy gap for superconductors with strong coupling," Journal of Low Temperature Physics, vol. 18, no. 34, pp. 241-271, 1975.

[634] J. P. Carbotte and C. Jiang, "Strong-coupling effects in $d$-wave superconductors," Physical Review B, vol. 48, no. 6, pp. 42314234, 1993.

[635] R. Combescot and X. Leyronas, "A simple theory for high $\Delta / T_{c}$ ratio in $d$-wave superconductors," European Physical Journal B, vol. 23, no. 2, pp. 159-163, 2001.

[636] D. C. Mattis and M. Molina, "Ratio $2 \Delta_{0} / k T_{c}$ in superconductivity," Physical Review B, vol. 44, no. 22, pp. 12565-12566, 1991.

[637] P. Udomsamuthirun, S. Ratanaburi, N. Saentalard, and S. Yoksan, "The ratio $2 \Delta_{0} / T_{c}$ in BCS superconductivity," Journal of Superconductivity, vol. 9, no. 6, pp. 603-604, 1996.

[638] R. J. Radtke, K. Levin, H.-B. Schüttler, and M. R. Norman, "Role of Van Hove singularities and momentum-space structure in high-temperature superconductivity," Physical Review B, vol. 48, no. 21, pp. 15957-15965, 1993.

[639] D. Einzel and I. Schürrer, "Weak coupling theory of clean $(d+s)$-wave superconductors," Journal of Low Temperature Physics, vol. 117, no. 1-2, pp. 15-52, 1999.

[640] H. Padamsee, J. E. Neighbor, and C. A. Shiffman, "Quasiparticle phenomenology for thermodynamics of strongcoupling superconductors," Journal of Low Temperature Physics, vol. 12, no. 3-4, pp. 387-411, 1973.

[641] I. Tifrea, I. Grosu, and M. Crisan, "Pseudogap influence on the $2 \Delta_{0} / T_{c}$ ratio in $d$-wave superconductors," Physica $C$, vol. 371, no. 2, pp. 104-110, 2002.

[642] N. Miyakawa, P. Guptasarma, J. F. Zasadzinski, D. G. Hinks, and K. E. Gray, "Strong dependence of the superconducting gap on oxygen doping from tunneling measurements on $\mathrm{Bi}_{2} \mathrm{Sr}_{2} \mathrm{CaCu}_{2} \mathrm{O}_{8-\delta}$," Physical Review Letters, vol. 80, no. 1, pp. 157-160, 1998.

[643] C. Pasquier, P. Auban-Senzier, T. Vuletic, S. Tomic, M. Heritier, and D. Jérome, "Coexistence of superconductivity and spin density wave orderings in Bechgaard and Fabre salts," Journal de Physique, vol. 12, no. 9, pp. 197-200, 2002.

[644] D. Jérome, "The development of organic conductors: organic superconductors," Solid State Sciences, vol. 10, no. 12, pp. 1692-1700, 2008.

[645] J. R. Jeffries, N. P. Butch, B. T. Yukich, and M. B. Maple, "Competing ordered phases in $\mathrm{URu}_{2} \mathrm{Si}_{2}$ : hydrostatic pressure and rhenium substitution," Physical Review Letters, vol. 99, no. 21, Article ID 217207, 4 pages, 2007.

[646] J. A. Janik, H. D. Zhou, Y.-J. Jo, et al., "Itinerant spin excitations near the hidden order transition in $\mathrm{URu}_{2} \mathrm{Si}_{2}$," Journal of Physics: Condensed Matter, vol. 21, no. 19, Article ID 192202, 4 pages, 2009.

[647] S. Elgazzar, J. Rusz, M. Amft, P. M. Oppeneer, and J. A. Mydosh, "Hidden order in $\mathrm{URu}_{2} \mathrm{Si}_{2}$ originates from Fermi surface gapping induced by dynamic symmetrybreaking," Nature Materials, vol. 8, no. 4, pp. 337-341, 2009.

[648] G. C. Psaltakis and E. W. Fenton, "Superconductivity and spin-density waves: organic superconductors," Journal of Physics C, vol. 16, no. 20, pp. 3913-3932, 1983.
[649] E. W. Fenton, "Cooper pairing in the presence of inhomogeneous magnetism," Progress of Theoretical Physics, no. 80, pp. 94-102, 1984.

[650] A. M. Gabovich and A. I. Voitenko, "Non-stationary Josephson tunneling involving superconductors with spin-density waves," Physica C, vol. 329, no. 3, pp. 198-230, 2000.

[651] T. T. M. Palstra, A. A. Menovsky, J. V. D. Berg, et al., "Superconducting and magnetic transitions in the heavyfermion system $\mathrm{URu}_{2} \mathrm{Si}_{2}$," Physical Review Letters, vol. 55, no. 24, pp. 2727-2730, 1985.

[652] A. J. A. de Oliveira and P. C. de Camargo, "Spin-density waves and charge-density waves in Cr alloys," in Handbook of Advanced Magnetic Materials Bolume IV: Advanced Magnetic Materials: Properties and Applications, Y. Liu, D. J. Sellmyer, and D. Shindo, Eds., p. 159, Springer, Heidelberg, Germany, 2006.

[653] R. Jaramillo, Y. Feng, J. C. Lang, et al., "Breakdown of the Bardeen-Cooper-Schrieffer ground state at a quantum phase transition," Nature, vol. 459, no. 7245, pp. 405-409, 2009.

[654] H. Chen, Y. Ren, Y. Qiu, et al., "Coexistence of the spin-density wave and superconductivity in $\mathrm{Ba}_{1-x} \mathrm{~K}_{x} \mathrm{Fe}_{2} \mathrm{As}_{2}$," Europhysics Letters, vol. 85, no. 1, Article ID 17006, 5 pages, 2009.

[655] K. Terashima, Y. Sekiba, J. H. Bowen, et al., "Fermi surface nesting induced strong pairing in iron-based superconductors," Proceedings of the National Academy of Sciences of the United States of America, vol. 106, no. 18, pp. 7330-7333, 2009.

[656] P. M. R. Brydon and C. Timm, "Theory of the excitonic spindensity-wave state in iron pnictides," Physical Review B, vol. 79, no. 18, Article ID 180504, 4 pages, 2009.

[657] S. A. J. Kimber, A. Kreyssig, Y.-Z. Zhang, et al., "Similarities between structural distortions underpressure and chemical doping in superconducting $\mathrm{BaFe}_{2} \mathrm{As}_{2}$," Nature Materials, vol. 8, no. 6, pp. 471-475, 2009.

[658] M. M. Korshunov and I. Eremin, "Theory of magnetic excitations in iron-based layered superconductors," Physical Review B, vol. 78, no. 14, Article ID 140509, 4 pages, 2008.

[659] I. Dimov, P. Goswami, X. Jia, and S. Chakravarty, "Competing order, Fermi surface reconstruction, and quantum oscillations in underdoped high-temperature superconductors," Physical Review B, vol. 78, no. 13, Article ID 134529, 14 pages, 2008.

[660] B. Pradhan and G. C. Rout, "Antiferromagnetic and superconducting gaps of some superconductors," Physica C, vol. 468, no. 1, pp. 72-80, 2008.

[661] A. D. Beyer, C.-T. Chen, M. S. Grinolds, M. L. Teague, and N.-C. Yeh, "Competing orders and the doping and momentum dependent quasiparticle excitations in cuprate superconductors," Physica C, vol. 468, no. 6, pp. 471-479, 2008.

[662] A. B. Vorontsov, M. G. Vavilov, and A. V. Chubukov, "Interplay between magnetism and superconductivity in the iron pnictides," Physical Review B, vol. 79, no. 6, Article ID 060508, 4 pages, 2009.

[663] A. N. Lavrov, L. P. Kozeeva, M. R. Trunin, and V. N. Zverev, "Competition and coexistence of antiferromagnetism and superconductivity in $R^{2} \mathrm{Ba}_{2} \mathrm{Cu}_{3} \mathrm{O}_{6+x}(R=\mathrm{Lu}, \mathrm{Y})$ single crystals," Physical Review B, vol. 79, no. 21, Article ID 214523, 6 pages, 2009.

[664] A. A. Gorbatsevich and Yu. V. Kopaev, "Magnetic state with orbital toroidal ordering and superdiamagnetism," in Superconductivity, Superdiamagnetism, Superfluidity, V. L. Ginzburg, Ed., pp. 175-241, Mir, Moscow, Russia, 1987. 

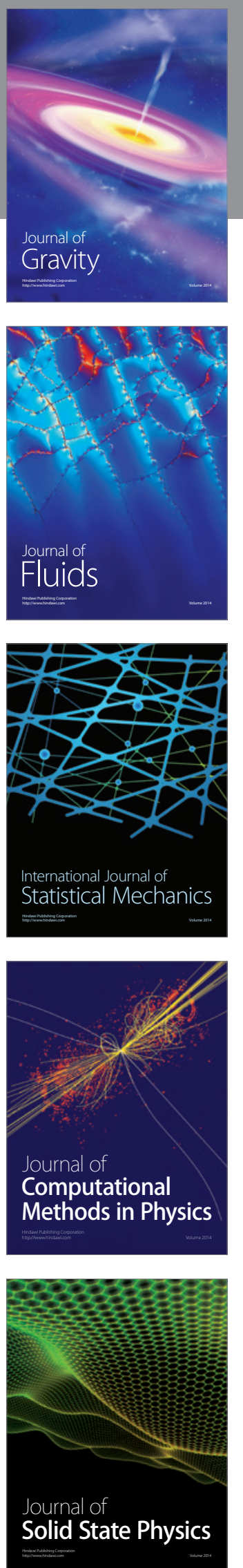

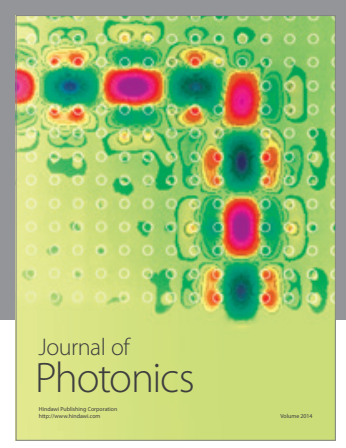

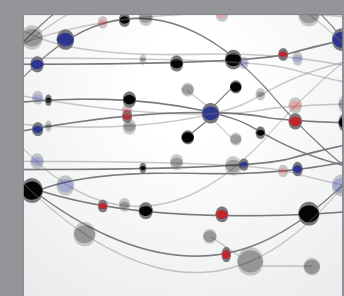

The Scientific World Journal
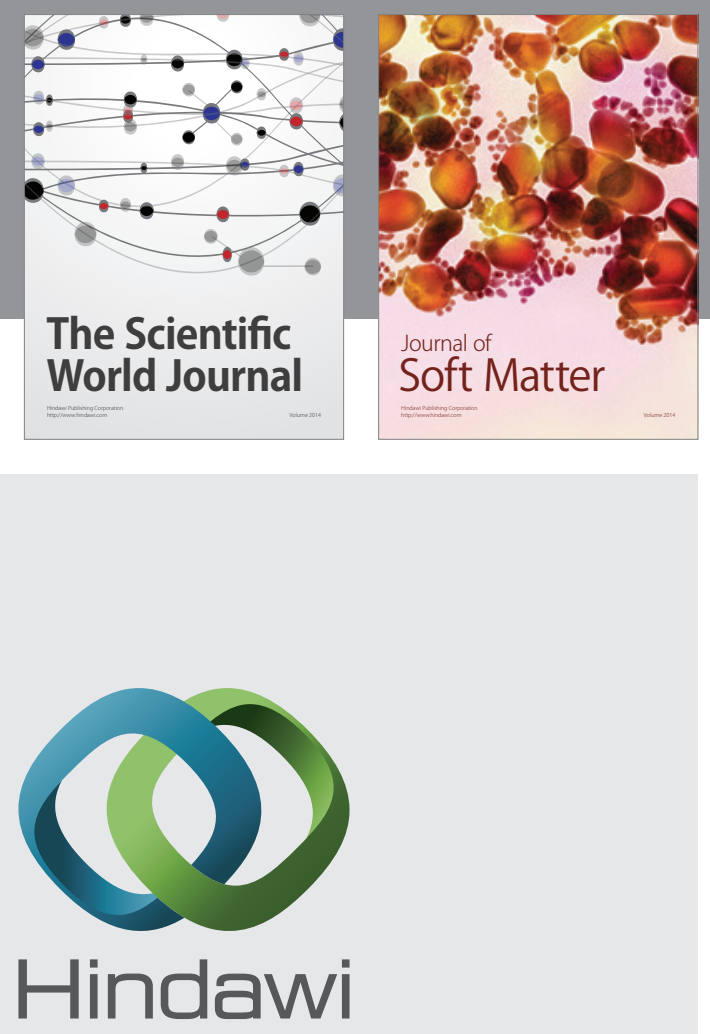

Submit your manuscripts at

http://www.hindawi.com
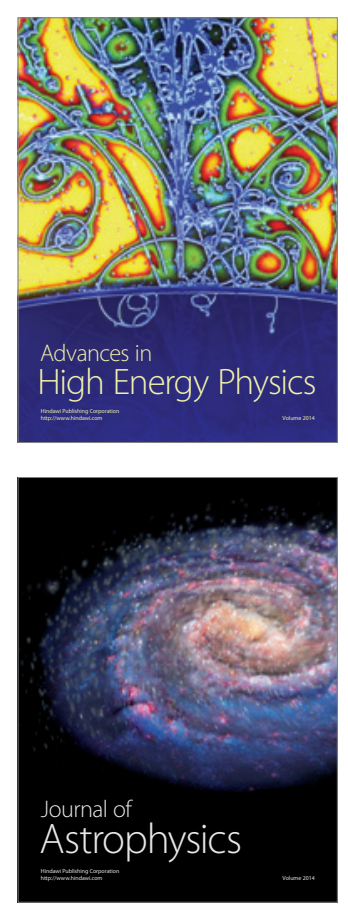
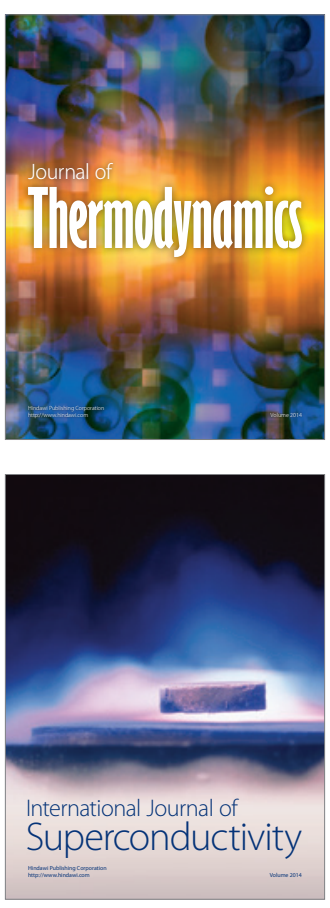
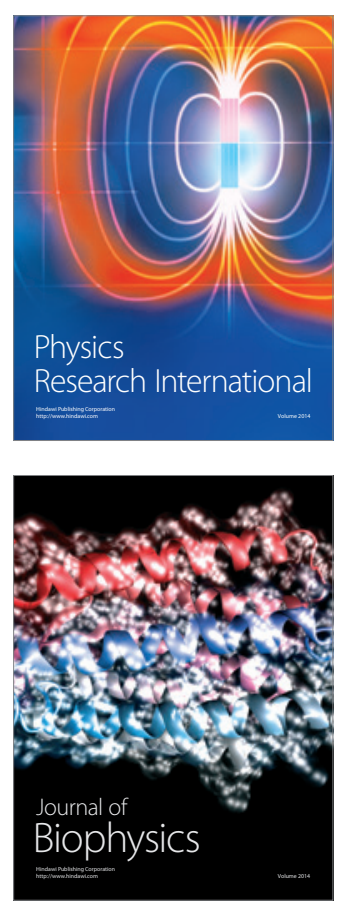
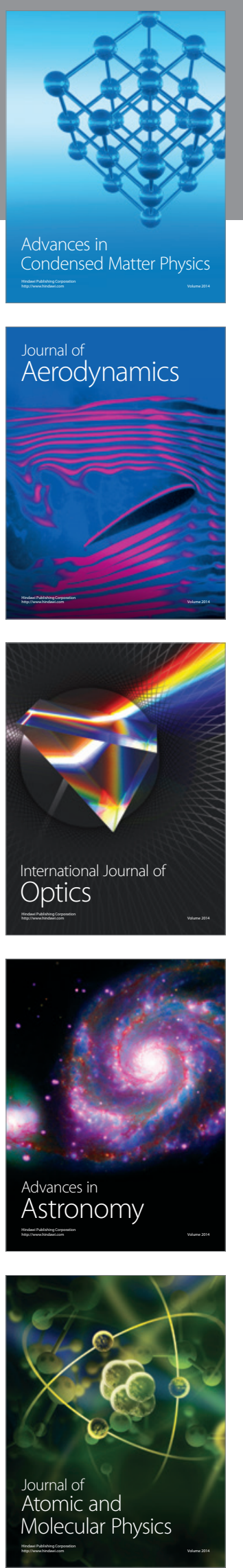